\title{
Implementation of the vortex force formalism in the Coupled Ocean- Atmosphere-Wave-Sediment Transport (COAWST) modeling system for inner shelf and surf zone applications
}

\author{
Nirnimesh Kumar ${ }^{1 *}$, George Voulgaris ${ }^{1}$ \\ ${ }^{1}$ Department of Earth and Ocean Sciences \\ Marine Science Program \\ University of South Carolina \\ Columbia, SC 29208, USA \\ nkumar@geol.sc.edu; Ph: 803-777-4504; \\ gvoulgaris@geol.sc.edu; Ph: 803-777-2549 \\ *Corresponding Author
}

\author{
John C. Warner ${ }^{2}$, Maitane Olabarrieta ${ }^{2}$ \\ ${ }^{2}$ U.S. Geological Survey \\ Coastal and Marine Geology Program \\ 384 Woods Hole Road \\ Woods Hole, MA 02543 \\ jcwarner@usgs.gov; Ph: 508-457-2237; \\ molabarrieta@usgs.gov; Ph: 508-457-2308
}

\begin{abstract}
The coupled ocean-atmosphere-wave-sediment transport modeling system (COAWST) enables simulations that integrate oceanic, atmospheric, wave and morphological processes in the coastal ocean. Within the modeling system, the three-dimensional ocean circulation module (ROMS) is coupled with the wave generation and propagation model (SWAN) to allow full integration of the effect of waves on circulation and vice versa. The existing wave-current coupling component utilizes a depth dependent radiation stress approach. In here we present a new approach that uses the vortex force formalism. The formulation adopted and the various parameterizations used in the model as well as their numerical implementation are presented in detail. The performance of the new system is examined through the presentation of four test cases. These include obliquely incident waves on a synthetic planar beach and a natural barred beach (DUCK' 94); normal incident waves on a nearshore barred morphology with rip channels; and wave-induced mean flows outside the surf zone at the Martha's Vineyard Coastal Observatory (MVCO).

Model results from the planar beach case show good agreement with depth-averaged analytical solutions and with theoretical flow structures. Simulation results for the DUCK' 94 experiment agree closely with measured profiles of cross-shore and longshore velocity data from Garcez-Faria et al. (1998, 2000). Diagnostic simulations showed that the nonlinear processes of wave roller generation and wave-induced mixing are important for the accurate simulation of surf zone flows. It is further recommended that a more realistic approach for determining the contribution of wave rollers and breaking induced turbulent mixing can be formulated using nondimensional parameters which are functions of local wave parameters and the beach slope. Dominant terms in the cross-shore momentum balance are found to be the quasi-static pressure gradient and breaking acceleration. In the alongshore direction, bottom stress, breaking acceleration, horizontal advection and horizontal vortex forces dominate the momentum balance. The simulation results for the bar / rip channel morphology case clearly show the ability of the modeling system to reproduce horizontal and vertical circulation patterns similar to those found in laboratory studies and to numerical simulations using the radiation stress representation. The vortex force term is found to be more important at locations where strong flow vorticity interacts with the wave-induced Stokes flow field. Outside the surf zone, the three-dimensional model
\end{abstract}


46 simulations of wave-induced flows for non- breaking waves closely agree with flow observations

47 from MVCO, with the vertical structure of the simulated flow varying as a function of the

48 vertical viscosity as demonstrated by Lentz et al. (2008).

49

Keywords: vortex-force, wave-current interaction, COAWST, ROMS, SWAN, radiation stress, three-dimensional, modeling, rip current, littoral velocities, nearshore circulation, bottom streaming 


\section{Introduction}

Coupling of rapidly oscillating surface gravity waves to slowly varying currents creates unique flow patterns in both inner shelf and surf zone environments. The effect of mean currents on surface gravity waves is exhibited as a Doppler shift in wave frequency, accompanied with a change in phase speed. Conversely, the effect of rapidly oscillating surface gravity waves on mean flow is manifested through the provision of additional momentum and mass flux to the mean flow. This coupling is usually accommodated by averaging the fast oscillations over longer time scales and provides a mechanism for the inclusion of the so called Wave Effect on Currents (WEC).

Wave-current interaction can be expressed in an Eulerian reference frame by assuming an analytic continuation of the wave-induced velocities above the air-sea interface (e.g., Garrett, 1976; McWilliams et al., 2004; Smith, 2006; Newberger and Allen, 2007a). A Generalized Lagrangian Mean (GLM) Framework (Andrews and McIntyre, 1978ab; Ardhuin et al., 2008) provides a formulation which is generally equivalent to the Eulerian mean, with a physical interpretation of the velocity above the wave trough level as a quasi-Eulerian velocity. Finally, the alternate Generalized Lagrangian Mean framework (Andrews and McIntyre, 1978a; Groeneweg and Klopman, 1998; Ardhuin et al., 2008b) is a distinct approach which works with the Lagrangian mean velocity. This may also be obtained using a vertically moving average (Mellor, 2003; 2005; 2008; 2011). The prognostic variables in Eulerian and Lagrangian mean flow equations are the quasi-Eulerian mean and Lagrangian mean velocity, respectively. The quasi-Eulerian mean velocity is the difference between Lagrangian mean velocity and the wave pseudo-momentum (e.g., Jenkins, 1989; Bennis et al., 2011). A detailed description of available Eulerian, GLM and "alternative" GLM averaging techniques along with their advantages and disadvantages in identifying the role of fast oscillations on mean circulation can be found in Ardhuin et al. (2008) and Bennis et al. (2011). In the present work we concentrate on the threedimensional momentum equations and their representation in an Eulerian reference frame.

The terms corresponding to WEC in the mean flow equations can be represented as the gradient of radiation stress tensor or as Vortex Force (VF, Craik and Leibovich, 1976). The radiation stress tensor is defined as the flux of momentum due to surface gravity waves (Longuet-Higgins and Stewart, 1964), while the VF representation splits the wave-averaged effects into gradients of a Bernoulli head and a vortex force. The Bernoulli head is an adjustment of pressure in accommodating incompressibility (Lane et al., 2007), while, after wave averaging, the vortex force is a function of wave-induced Stokes drift and flow vorticity.

Recently, a number of three-dimensional, hydrostatic ${ }^{1}$ ocean models have been developed and used to study wave-current interaction. Newberger and Allen (2007a) using an Eulerian framework, added wave forcing in form of surface and body forces, and depth- averaged VF terms in the Princeton Ocean Model (POM), which has evolved into "Nearshore POM". McWilliams et al. (2004, hereinafter MRL04) developed a multi-scale asymptotic theory for the evolution and interaction of currents and surface gravity waves of finite depth. This method

\footnotetext{
${ }^{1}$ Non-hydrostatic ocean models have also been developed and used to study wave-current interaction. The models resolving non-hydrostatic pressure have been used to study propagation of shortwaves on a deep basin, internal waves and tides (Kanarska et al., 2007), internal solitary wave shoaling and breaking, lock-exchange problem (Lai et al., 2010), and wave propagation, shoaling and breaking in the surf zone (Zijlema et al., 2011). Nevertheless, most of the model simulations conducted using non-hydrostatic models are validated against laboratory test cases, while hydrostatic ocean models are utilized for majority of realistic nearshore field experiments.
} 
separates currents, long waves and surface gravity waves on the basis of differences in their spatial and temporal variation, as a function of the wave slope. The work of MRL04 was implemented in UCLA ROMS and extended for applications within the surf zone by Uchiyama et al. (2010, hereinafter U10).

The VF formalism based model presented by U10 separates the effects of wave forcing into conservative (Bernoulli head and vortex force) and non-conservative (wave dissipation induced acceleration) contributions. A separation between conservative and non-conservative forces is pertinent as the former has a known vertical distribution, while the latter can presently only be expressed with an empirical vertical profile. U10 presented the non-conservative wave forcings with a depth-limited wave dissipation calculated using the formulations of Thornton and Guza (1983) and Church and Thornton (1993), bottom streaming and a wave roller model based on Reniers et al. (2004a). The turbulence closure model is K-profile parameterization (KPP) with additional mixing due to wave breaking following Apotsos et al. (2007). The circulation model is coupled to a spectrum-peak WKB (Wentzel Kramer Brillouin) wave refraction model.

The methodology presented by MRL04 and U10 can be extrapolated for modeling with nesting components that requires seamless simulation of processes simulated at a variety of scales for water depths from the deep ocean to the surf zone. Such applications include the development of rip current prediction system (e.g., Voulgaris et al., 2011), sediment transport studies (e.g., Kumar et al., 2011b), and nearshore water quality prediction systems (Grant et al., 2005) amongst others. Thus it is imperative that these types of models are made available to the scientific community through the upgrade of existing public domain modeling systems. As a primary step in this direction we implement the VF approach to the three-dimensional ocean model ROMS, coupled with the wave model Simulating Waves Nearshore (SWAN), within the framework of the COAWST modeling system (Warner et al., 2010). This modeling system allows for simulating wave driven flows and sediment transport in the intertidal region (see Kumar et al., 2011b) through wetting and drying algorithms, a capability not available in the U10 model implementation. Furthermore, the U10 model uses a KPP parameterized mixing scheme that fails to accurately represent the mixing in bottom boundary layer and in nearshore regions where the surface and bottom boundary layer interact (Durski et al., 2004).

The implementation of VF formalism into the COAWST modeling system is conducted with significant modifications to the method of U10 which includes: (a) Enhanced mixing implementation using the Generic Length Scale (GLS) scheme with the addition of waveinduced mixing in the form of surface boundary condition (Feddersen and Trowbridge, 2005). (b) Improved vertical structure of depth-limited wave dissipation induced acceleration that scales with the wave height. This $\cosh$-based distribution is limited to the surface layer in deeper water, while in shallow waters (where the water depth is similar to wave height) the dissipation effect is delivered to the depth of water column influenced by wave propagation (c) Improved implementation of wave dissipation input which is provided by the wave driver model directly rather than being estimated locally using empirical formulations (Thornton and Guza, 1983; Church and Thornton, 1993); (d) Incorporation of bottom streaming using multiple formulations and wave-induced tangential flux at the surface as an option. The objectives of this manuscript are: (a) to describe the implementation of the VF formalism; (b) validate the model using analytical, laboratory and field observations applicable to both surf zone and inner shelf environments; and (c) provide a set of standard test cases for model comparisons.

The versatility and general applicability of the model presented here are demonstrated over a number of cases that not only include commonly used case (see U10, Newberger and 
Allen, 2007b) of obliquely incident waves on a planar and barred beach, but extend beyond to include applications of the model for the study of complex flow regimes developed in a nearshore barred beach with rip channels as well as for the study of wave-induced flow fields outside the surf zone in the inner shelf. We compare depth-averaged flow fields from VF representation to those obtained using a two-dimensional numerical model based on radiation stress representation, and identify the role of different forcing terms. A comparison to threedimensional flows from a radiation stress representation has been avoided in absence of any selfconsistent theory (see Ardhuin et al., 2008b; Bennis et al., 2011; Kumar et al., 2011a) for the same.

The outline of the paper is as follows. The model formulation is presented in section 2, while its numerical implementation is described in section 3 . The new model capabilities are demonstrated in section 4 through presentation of four test cases that cover both surf zone and inner shelf processes: (a) obliquely incident waves on a synthetic planar beach; (b) obliquely incident waves on a natural, sandy, barred beach (DUCK' 94 experiment); (c) nearshore barred morphology with rip channels; and (d) structure of undertow observed on the inner shelf. Discussion on differences and similarities of flow structure derived by expressing WEC using $\mathrm{VF}$ and radiation stress representations are shown in section 5 followed by a summary and conclusion section.

\section{Model Formulation}

The ocean component of COAWST is the Regional Ocean Modeling System (ROMS), a three-dimensional, free surface, topography following numerical model, which solves finite difference approximation of Reynolds Averaged Navier Stokes (RANS) equations using hydrostatic and Boussinesq approximation with a split explicit time stepping algorithm (Shchepetkin and McWilliams, 2005; Haidvogel et al., 2008; Shchepetkin and McWilliams, 2009). ROMS includes several options for several model capabilities, such as various advection schemes (second, third and fourth order) and turbulence closure models (e.g., Generic Length Scale mixing, Mellor-Yamada, Brunt-Väisälä frequency mixing, user provided analytical expression and K-profile parameterization). As Shchepetkin and McWilliams (2009) state, currently there are four variations of ROMS-family codes. In this contribution we use a version based on the Rutgers University ROMS which was first introduced by Haidvogel et al. (2000) and subsequently any reference to ROMS denotes this particular version.

\subsection{VF Equations}

The VF approach was implemented following the conventions described in MRL04 and based on the formulation as presented in U10, with several key modifications that are applicable for this particular modeling system. Terms corresponding to wave effect on current are assembled and shown on the right hand side of the equations presented below. Boldface typesets are used for horizontal vectors only, while the vertical component is represented by a normal typeset so that a three-dimensional current vector is designated as $(\mathbf{u}, \mathbf{w})$. Following the above conventions the model equations can be written as:

$$
\frac{\partial \mathbf{u}}{\partial t}+\left(\mathbf{u} \cdot \nabla_{\perp}\right) \mathbf{u}+w \frac{\partial \mathbf{u}}{\partial z}+f \hat{z} \times \mathbf{u}+\nabla_{\perp} \varphi-\mathbf{F}-\mathbf{D}+\frac{\partial}{\partial z}\left(\overline{\boldsymbol{u}^{\prime} w^{\prime}}-v \frac{\partial \boldsymbol{u}}{\partial z}\right)=-\nabla_{\perp} \mathcal{K}+\mathbf{J}+\mathbf{F}^{w}
$$




$$
\begin{gathered}
\frac{\partial \varphi}{\partial z}+\frac{g \rho}{\rho_{0}}=-\frac{\partial \mathcal{K}}{\partial \mathbf{z}}+K \\
\nabla_{\perp} \cdot \mathbf{u}+\frac{\partial w}{\partial z}=0 \\
\frac{\partial c}{\partial t}+\left(\mathbf{u} \cdot \nabla_{\perp}\right) c+w \frac{\partial c}{\partial z}-C_{\text {source }}+\frac{\partial}{\partial z}\left(\overline{c^{\prime} w^{\prime}}-v_{\theta} \frac{\partial c}{\partial z}\right)=-\left(\mathbf{u}^{S t} \cdot \nabla_{\perp}\right) c-w^{S t} \frac{\partial c}{\partial z}+\frac{1}{2} \frac{\partial}{\partial z}\left[\mathrm{C} \frac{\partial c}{\partial z}\right]
\end{gathered}
$$

where $(\mathbf{u}, \mathbf{w})$ and $\left(\mathbf{u}^{\mathrm{St}}, \mathrm{w}^{\mathrm{St}}\right)$ are Eulerian mean and Stokes velocities, respectively. At this stage it is pertinent to point out that the velocities presented in this paper are the quasi-Eulerian mean velocities. This velocity is defined as the Lagrangian mean velocity minus the Stokes drift (Jenkins, 1989). Below the wave troughs it is very nearly equal to the one that is measurable by a fixed current meter. Above the wave troughs, it provides a smooth extension of the velocity profile all the way to the mean sea level, as assumed in the MRL04 theory. For consistency purposes with the notation of MRL04 and U10, in the remaining of the paper these quasiEulerian mean velocities are referred to as Eulerian mean velocities. $f$ is the Coriolis parameter, $\varphi$ is the dynamic pressure (normalized by the density $\rho_{0}$ ); $\mathbf{F}$ is the non-wave, non-conservative force; D represents the diffusive terms (viscosity and diffusion); $(\mathbf{J}, K)$ is the VF and $\mathcal{K}$ is the lower order Bernoulli head as described in MRL04 (see Sec. 9.6 in MRL04); $\mathbf{F}^{w}$ is the sum of momentum flux due to all non-conservative wave forces; $\mathrm{c}$ is any material tracer concentration; $C_{\text {source }}$ are tracer source/sink terms and $E$ is the wave-induced tracer diffusivity. An overbar indicates time average, and a prime indicates turbulent fluctuating quantity. $\rho$ and $\rho_{0}$ are total and reference densities of sea water; $g$ is the acceleration due to gravity; and $v$ and $v_{\theta}$ are the molecular viscosity and diffusivity, respectively. The vertical coordinate $(z)$ range varies from $h(x) \leq z \leq \zeta+\hat{\zeta}$, where $\zeta$ and $\hat{\zeta}$, are the mean and quasi-static sea (wave-averaged) level components, respectively. All wave quantities are referenced to a local wave-averaged sea level, $z=\zeta+\hat{\zeta}$.

The three-dimensional Stokes velocity $\left(\mathbf{u}^{\mathrm{St}}, \mathrm{w}^{\mathrm{St}}\right)$ is defined for a spectral wave-field as:

$$
\begin{gathered}
\boldsymbol{u}^{S t}(z)=\frac{2 E}{c} \frac{\cosh [2 \mathcal{Z}]}{\sinh [2 \mathcal{H}]} \boldsymbol{k} \\
w^{S t}(z)=-\nabla_{\perp} \cdot \int_{-\boldsymbol{h}}^{z} \boldsymbol{u}^{S t} d z^{\prime}
\end{gathered}
$$

where $h(x)$ is the resting depth; $E$ is the wave energy; $c$ is the phase speed of the waves; $\boldsymbol{k}$ is the wave number vector and $\mathrm{k}$ is its magnitude, and $\mathcal{H}$ and $\mathcal{Z}$ are the normalized vertical lengths defined as:

$$
\mathcal{H}=k(h+\zeta+\zeta)=k D ; \text { and } \mathcal{Z}=k(z+h)
$$


where $D=(h+\zeta+\zeta)$ is the wave-averaged thickness of the water column. Finally, the wave energy $(E)$, phase speed (c), and intrinsic frequency $(\sigma)$ are given by:

$$
\begin{gathered}
E=\frac{1}{8} g H^{2} \\
c=\frac{\sigma}{k} \\
\sigma=\sqrt{g \cdot k \cdot \tanh [\mathcal{H}]}
\end{gathered}
$$

where $H$ is the root mean square wave height.

The $\operatorname{VF}(\mathbf{J}, K)$ and the Bernoulli head $(\mathcal{K})$ terms shown in Eqn. 1 are expressed as:

$$
\begin{gathered}
\mathbf{J}=-\hat{z} \times \mathbf{u}^{s t}\left(\left(\hat{z} \cdot \nabla_{\perp} \times \mathbf{u}\right)+f\right)-w^{s t} \frac{\partial \mathbf{u}}{\partial z} \\
\mathrm{~K}=\mathbf{u}^{s t} \cdot \frac{\partial u}{\partial z} \\
\mathcal{K}=\frac{\sigma H^{2}}{16 \cdot k \sinh ^{2}[\mathcal{H}]} \int_{-h}^{z} \frac{\partial^{2} \mathcal{V}}{\partial z^{\prime 2}} \sinh \left[2 k\left(z-z^{\prime}\right)\right] d z^{\prime}
\end{gathered}
$$

where $\mathcal{V}=\mathbf{k} . \boldsymbol{u}$, and $\hat{z}$ is the unit vector in vertical direction. Physically, the Bernoulli head term represents an adjustment in the mean pressure to accommodate for the presence of waves (Lane et al., 2007) and the VF terms represent an interaction between Stokes drift and vorticity of the mean flow.

The wave-induced tracer diffusivity in Eqn. 1 is defined as:

$$
€=\frac{1}{8} \frac{\partial}{\partial t}\left(\frac{H \sinh [\mathcal{Z}]}{\sinh [\mathcal{H}]}\right)
$$

while the quasi-static sea level component is given by:

$$
\hat{\zeta}=\frac{-P_{a t m}}{g \rho_{0}}-\frac{H^{2} k}{8 \cdot \sinh [2 \mathcal{H}]}
$$

The term $\hat{\zeta}$ contains inverse barometric response due to changes in atmospheric pressure $\left(P_{a t m}\right)$ and a wave-averaged set-up/set-down (with respect to the still water).

It is important to note that in the above formulations the total contribution of Bernoulli head has been separated in two parts of different order each. The higher order contribution is a quasi-static balance between mean pressure, mean surface elevation and the wave stresses, which is absorbed here as a part of the quasi-static sea level component $(\hat{\zeta})$ in Eqn. 7. The lower order quasi-static balance has been expressed as $\mathcal{K}$ in Eqn. 5 .

When using a spectral wave model such as SWAN, wave height $\left(H=H_{s i g} / \sqrt{2}\right)$ and bottom orbital velocity $\left(u_{o r b}\right)$ values are provided after integrating the energy over all frequencies and directions, while wavenumber $(k)$, wave direction and, frequency $(f)$ are those corresponding to the peak frequency. Wave dissipation due to depth-limited breaking, whitecapping and bottom friction is also provided by SWAN. 


\subsection{Boundary Conditions}

The kinematic and pressure boundary conditions are given as:

$$
\begin{gathered}
\left.w\right|_{-h}+\left.\mathbf{u}\right|_{-h} \cdot \nabla_{\perp} h=0 \\
\left.w\right|_{\zeta+\hat{\zeta}}-\frac{\partial \zeta}{\partial t}-\left(\left.\mathbf{u}\right|_{\zeta+\hat{\zeta}} \cdot \nabla_{\perp}\right) \zeta=\nabla_{\perp} \cdot \overline{\mathbf{U}}^{S t}+\frac{\partial \hat{\zeta}}{\partial t}+\left(\left.\mathbf{u}\right|_{\zeta+\hat{\zeta}} \cdot \nabla_{\perp}\right) \hat{\zeta} \\
g \zeta-\left.\varphi\right|_{\zeta+\hat{\zeta}}=g \mathcal{P}
\end{gathered}
$$

where $\overline{\mathbf{U}}^{S t}$ is the depth-averaged Stokes velocity and $\mathcal{P}$ is the wave-averaged forcing surface boundary condition (see section 9.3 in MRL04) defined as:

$$
\mathcal{P}=\frac{H^{2}}{8 \sigma}\left\{\begin{array}{c}
\frac{\tanh [\mathcal{H}]}{\sinh [2 \mathcal{H}]}\left(-\left.\frac{\partial \mathcal{V}}{\partial z}\right|_{\zeta+\hat{\zeta}}+\left.\cosh [2 \mathcal{H}] \frac{\partial \mathcal{V}}{\partial z}\right|_{-h}+\int_{-h}^{\zeta+\hat{\zeta}} \frac{\partial^{2} \mathcal{V}}{\partial z^{\prime 2}} \cosh \left[2 k z^{\prime}\right] d z^{\prime}\right) \\
-\left.2 k \tanh [\mathcal{H}] \mathcal{V}\right|_{\zeta+\hat{\zeta}}
\end{array}\right\}
$$

The equations corresponding to barotropic mode have not been presented here for brevity, although details can be found in U10.

\section{Numerical implementation}

For implementation into the modeling system, the equations presented in section 2 are expressed in a mass flux form. The wave-induced terms are no longer retained to the right hand side and the lower order Bernoulli head becomes part of the dynamic pressure.

First we define the following quantities:

$$
\begin{aligned}
\left(\mathbf{u}^{l}, \omega^{l}\right) & =(\mathbf{u}, \omega)+\left(\mathbf{u}^{S t}, \omega^{S t}\right) \\
\zeta^{c} & =\zeta+\hat{\zeta} \\
\varphi^{c} & =\varphi+\mathcal{K}
\end{aligned}
$$

where $\zeta^{c}$ is the composite sea level, $\varphi^{c}$ is the sum of Bernoulli head and the dynamic pressure, while to lowest order the Lagrangian mean velocity $\left(\mathbf{u}^{l}, \omega^{l}\right)$ is represented as the sum of Stokes $\left(\mathbf{u}^{S t}, \omega^{S t}\right)$ and Eulerian mean velocities $(\mathbf{u}, \omega) . \omega_{s}$ is the vertical Eulerian mean velocity in a sigma coordinate system.

The continuity and momentum balance equations in horizontal $(\xi, \eta)$ orthogonal curvilinear and vertical $(s)$ terrain following coordinate system are:

$$
\frac{\partial}{\partial t}\left(\frac{H_{z}}{m n}\right)+\frac{\partial}{\partial \xi}\left(\frac{H_{z} u^{l}}{n}\right)+\frac{\partial}{\partial \eta}\left(\frac{H_{z} v^{l}}{m}\right)+\frac{\partial}{\partial s}\left(\frac{\omega_{s}^{l}}{m n}\right)=0
$$




$$
\begin{aligned}
& \frac{\partial}{\partial t}\left(\frac{H_{z}}{m n} u\right)+\frac{\partial}{\partial \xi}\left(\frac{H_{z} u}{n} u\right)+\frac{\partial}{\partial \eta}\left(\frac{H_{z} v}{m} u\right)+u \frac{\partial}{\partial \xi}\left(\frac{H_{z} u^{S t}}{n}\right)+u \frac{\partial}{\partial \eta}\left(\frac{H_{z} v^{S t}}{m}\right) \\
& \text { ACC } \\
& +\frac{\partial}{\partial s}\left(\frac{\omega_{s}}{m n} u\right)+u \frac{\partial}{\partial s}\left(\frac{\omega_{s}^{S t}}{m n}\right)-H_{z}\left(\frac{f v}{m n}\right)-H_{z}\left(\frac{f v^{s t}}{m n}\right)= \\
& \mathrm{VA} \stackrel{\mathrm{COR}}{\longrightarrow} \stackrel{\mathrm{StCOR}}{\longrightarrow} \\
& -\left.\frac{H_{z}}{n} \frac{\partial \varphi^{c}}{\partial \xi}\right|_{z}+H_{z} v^{s t}\left(\frac{1}{n} \frac{\partial v}{\partial \xi}-\frac{1}{m} \frac{\partial u}{\partial \eta}\right)-\omega_{s}^{S t} \frac{\partial}{\partial s}\left(\frac{u}{m n}\right) \\
& \mathrm{PG} \longrightarrow \mathrm{HVF} \\
& +\frac{H_{z} \mathcal{F}^{\xi}}{m n}+\frac{H_{z} \mathcal{F}^{w \xi}}{m n}+\frac{H_{z} D^{\xi}}{m n}-\frac{\partial}{\partial s}\left(\overline{u^{\prime} w^{\prime}}-\frac{v}{H_{z}} \frac{\partial u}{\partial s}\right)+\hat{\mathcal{F}}^{u}
\end{aligned}
$$

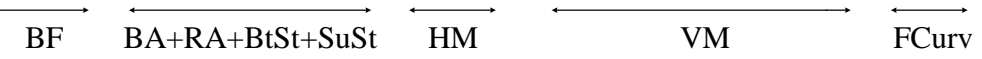

$$
\begin{aligned}
& \frac{\partial}{\partial t}\left(\frac{H_{z}}{m n} v\right)+\frac{\partial}{\partial \xi}\left(\frac{H_{z} u}{n} v\right)+\frac{\partial}{\partial \eta}\left(\frac{H_{z} v}{m} v\right)+v \frac{\partial}{\partial \xi}\left(\frac{H_{z} u^{S t}}{n}\right)+v \frac{\partial}{\partial \eta}\left(\frac{H_{z} v^{S t}}{m}\right) \\
& \text { ACC } \longrightarrow \text { HA } \\
& +\frac{\partial}{\partial s}\left(\frac{\omega_{s}}{m n} v\right)+v \frac{\partial}{\partial s}\left(\frac{\omega_{s}^{S t}}{m n}\right)+H_{z}\left(\frac{f u}{m n}\right)+H_{z}\left(\frac{f u^{s t}}{m n}\right)= \\
& \mathrm{VA} \stackrel{\mathrm{COR}}{\longrightarrow} \stackrel{\mathrm{StCOR}}{\mathrm{SOR}} \\
& -\left.\frac{H_{z}}{m} \frac{\partial \varphi^{c}}{\partial \eta}\right|_{z}-H_{z} u^{s t}\left(\frac{1}{n} \frac{\partial v}{\partial \xi}-\frac{1}{m} \frac{\partial u}{\partial \eta}\right)-\omega_{s}^{S t} \frac{\partial}{\partial s}\left(\frac{v}{m n}\right) \\
& \mathrm{PG} \longrightarrow \mathrm{HVF} \\
& +\frac{H_{z} \mathcal{F}^{\eta}}{m n}+\frac{H_{z} \mathcal{F}^{w \eta}}{m n}+\frac{H_{z} D^{\eta}}{m n}-\frac{\partial}{\partial s}\left(\overline{v^{\prime} w^{\prime}}-\frac{v}{H_{z}} \frac{\partial v}{\partial s}\right)+\hat{\mathcal{F}}^{v} \\
& \underset{\mathrm{BF}}{\mathrm{BA}+\mathrm{RA}+\mathrm{BtSt}+\mathrm{SuSt}} \stackrel{\mathrm{HM}}{\longrightarrow} \stackrel{\mathrm{VM}}{\longrightarrow}
\end{aligned}
$$


for the ' $\mathrm{x}$ ' and 'y' ( $\xi$ and $\eta$ ) directions, respectively; where $m^{-1}$ and $n^{-1}$ are the Lamé metric coefficients, and $H_{z}$ is the grid-cell thickness. The vertical sigma coordinates $s$ varies from -1 at the bottom to 0 at the free surface. $\mathbf{F}=\left(\mathcal{F}^{\xi}, \mathcal{F}^{\eta}\right)$ is the non-wave body force; $\left(D^{\xi}, D^{\eta}\right)$ represents the parameterized momentum mixing terms; and $\mathbf{F}^{w}=\left(\mathcal{F}^{w \xi}, \mathcal{F}^{w \eta}\right)$ is the momentum flux from non-conservative wave terms described in 3.1 below. In a Cartesian coordinate system $m$ and $n$ are unity and the curvilinear terms $\left(\hat{\mathcal{F}}^{u}, \hat{\mathcal{F}}^{v}\right)$ become zero.

In the momentum balance equations (Eqn. 11 and 12), the first term on the left hand side is the local acceleration (ACC), second to fifth terms constitute the horizontal advection (HA), sixth and seventh terms are vertical advection (VA), eighth and the ninth term represent Coriolis (COR) and Stokes-Coriolis (StCOR) forces, respectively. On the right hand side of the momentum balance equations, the first term is the pressure gradient (PG), and the combination of the second and the third term is the horizontal vortex force (HVF). The non-wave body force (BF) is the fourth term, while the contribution of breaking and roller acceleration, and bottom and surface streaming is represented collectively by the fifth term (BA+RA+BtSt+SuSt). Horizontal (HM) and vertical mixing (VM) are sixth and the seventh terms, respectively. The last term on the right hand side is the curvilinear metric term given by Eqn. 16 and 17.

The geopotential function derived after depth integrating the vertical momentum balance equation (see Eqn. 1) is:

$$
\varphi^{c}=g\left(\zeta^{c}-\hat{\zeta}\right)-\left.(g \mathcal{P}-\mathcal{K})\right|_{\zeta^{c}}+\int_{s}^{0}\left[\frac{g \rho}{\rho_{0}}-K\right] H_{z} d s
$$

The curvilinear terms $\hat{\mathcal{F}}^{u}$ and $\hat{\mathcal{F}}^{v}$ in Eqns. (11) and (12), respectively are:

$$
\begin{aligned}
\hat{\mathcal{F}}^{u}= & H_{z}\left[-u \frac{\partial}{\partial \eta}\left(\frac{1}{m}\right)+v \frac{\partial}{\partial \xi}\left(\frac{1}{n}\right)\right] v^{s t}+H_{z}\left[-u \frac{\partial}{\partial \eta}\left(\frac{1}{m}\right)+v \frac{\partial}{\partial \xi}\left(\frac{1}{n}\right)\right] v \\
& +H_{z}\left[-u^{S t} \frac{\partial}{\partial \eta}\left(\frac{1}{m}\right)+v^{S t} \frac{\partial}{\partial \xi}\left(\frac{1}{n}\right)\right] v
\end{aligned}
$$

$$
\begin{aligned}
\hat{\mathcal{F}}^{v}= & -H_{z}\left[-u \frac{\partial}{\partial \eta}\left(\frac{1}{m}\right)+v \frac{\partial}{\partial \xi}\left(\frac{1}{n}\right)\right] u^{s t}-H_{z}\left[-u \frac{\partial}{\partial \eta}\left(\frac{1}{m}\right)+v \frac{\partial}{\partial \xi}\left(\frac{1}{n}\right)\right] u \\
& -H_{z}\left[-u^{S t} \frac{\partial}{\partial \eta}\left(\frac{1}{m}\right)+v^{S t} \frac{\partial}{\partial \xi}\left(\frac{1}{n}\right)\right] u
\end{aligned}
$$

The vertical motion past sigma surfaces is given by:

$$
\omega_{s}^{l}=\left.\left[w^{l}-\left(\frac{\partial z}{\partial t}+\boldsymbol{u}^{l} \cdot \nabla_{\perp} z\right)\right]\right|_{s}
$$

The vertical mass flux through the sigma surfaces is calculated as:

$$
W^{l}=\int_{-1}^{s}\left(\frac{\partial U^{l}}{\partial \xi}+\frac{\partial V^{l}}{\partial \eta}\right) d s^{\prime}-\frac{1}{m n} \cdot \frac{z+h}{\zeta+h} \cdot \frac{\partial \zeta^{c}}{\partial t}
$$

where $U^{l}=H_{z} u^{l} / n, V^{l}=H_{z} v^{l} / m$, and $W^{l}=\omega_{s}^{l} /(m n)$ are grid-cell volume fluxes. 
The three-dimensional tracer equation is:

$$
\begin{gathered}
\frac{\partial}{\partial t}\left(\frac{H_{z}}{m n} c\right)+\frac{\partial}{\partial \xi}\left(\frac{H_{z} u}{n} c\right)+\frac{\partial}{\partial \eta}\left(\frac{H_{z} v}{m} c\right)+\frac{\partial}{\partial s}\left(\frac{\omega^{s}}{m n} c\right)=C_{\text {source }}-\frac{\partial}{\partial \xi}\left(\frac{H_{z} u^{S t}}{n} c\right) \\
-\frac{\partial}{\partial \eta}\left(\frac{H_{z} v^{S t}}{m} c\right)-\frac{\partial}{\partial s}\left(\frac{\omega_{s}^{S t}}{m n} c\right)-\frac{\partial}{\partial s}\left(\overline{c^{\prime} w^{\prime}}-\frac{v_{\theta}}{H_{z}} \frac{\partial v}{\partial s}\right)+\frac{\partial}{\partial s}\left(\frac{C}{H_{z}}\left(\frac{\partial c}{\partial s}\right)\right)
\end{gathered}
$$

where $C$ is the wave-induced tracer diffusivity as defined as in Eqn. 8.

Eqns. (11), (12) and (18) are closed by parameterization of the Reynolds stresses and turbulent tracer fluxes as:

$$
\overline{u^{\prime} w^{\prime}}=\frac{K_{M}}{H_{z}} \frac{\partial u}{\partial s} ; \overline{v^{\prime} w^{\prime}}=\frac{K_{M}}{H_{z}} \frac{\partial v}{\partial s} ; \overline{c^{\prime} w^{\prime}}=\frac{K_{H}}{H_{z}} \frac{\partial \rho}{\partial s}
$$

where $K_{M}$ is the momentum eddy viscosity and $K_{H}$ is the eddy diffusivity. Along with the kinematic and pressure boundary conditions (Eqn. 8), the surface wind and bottom stresses are prescribed as vertical boundary condition for the Reynolds stresses given as:

$$
\begin{aligned}
& \left.\frac{K_{M}}{H_{z}} \frac{\partial u}{\partial s}\right|_{s=0}=\tau_{s}^{\xi}(\xi, \eta, t) \\
& \left.\frac{K_{M}}{H_{z}} \frac{\partial v}{\partial s}\right|_{s=0}=\tau_{s}^{\eta}(\xi, \eta, t) \\
& \left.\frac{K_{M}}{H_{z}} \frac{\partial u}{\partial s}\right|_{s=-1}=\tau_{b}^{\xi}(\xi, \eta, t) \\
& \left.\frac{K_{M}}{H_{z}} \frac{\partial v}{\partial s}\right|_{s=-1}=\tau_{b}^{\eta}(\xi, \eta, t)
\end{aligned}
$$

where, $\boldsymbol{\tau}_{s}=\left(\tau_{s}^{\xi}, \tau_{s}^{\eta}\right)$ and $\boldsymbol{\tau}_{\boldsymbol{b}}=\left(\tau_{b}^{\xi}, \tau_{b}^{\eta}\right)$ are surface wind stress and bottom stress, respectively.

Although many different methods are available to incorporate bottom stress in ROMS (see Warner et al., 2008a), in the present application we use the simple quadratic drag method or the wave-current interaction method of Madsen (1994).

The horizontal momentum, continuity and tracer equations as well as the geopotential function along with the boundary conditions (Eqns. 10-20) are solved to obtain the Eulerian mean velocity $(\mathbf{u}, \omega)$ and composite sea level $\left(\zeta^{c}\right)$ as the prognostic variables.

The wave parameters required for calculating the Stokes velocities, WEC terms, and momentum flux from non-conservative wave forcing terms, $\mathbf{F}^{\mathbf{w}}$ (see next section for details) are provided through coupling to the wave model (SWAN). SWAN receives information about sea surface elevation, bathymetric change, and a circulation field from ROMS to determine the effect of currents and total water depth on wave propagation. In turn, ROMS receives information on surface and bottom wave parameters (height, orbital velocity, period, wavelength and direction), wave dissipation due to bottom friction, wave breaking, and whitecapping for non-conservative WEC processes. This exchange of information between the circulation and wave models occurs at user defined intervals in a two-way coupling scenario. One-way coupling of data feeding from wave to circulation model can be used if the impact of currents on wave field is negligible, or simply from the wave model to the ocean for processes such as enhanced bottom stress 
computations. A detailed discussion about model coupling can be found in Warner et al. (2008ab).

\subsection{Parameterization of non-conservative wave forcing, $\boldsymbol{F}^{w}$}

Waves propagating towards the shoreline lose energy through three different dissipation mechanisms: (a) bottom friction ( $\left.\epsilon^{b f}\right)$; (b) whitecapping ( $\epsilon^{\text {wcap }}$ ); and (c) depth-induced wave breaking $\left(\epsilon^{b}\right)$. The energy lost by these processes is included in the momentum balance (Eqns. 11 and 12) through the non-conservative wave forcing/acceleration term $\mathbf{F}^{\mathbf{w}}$ :

$$
\mathbf{F}^{w}\left(\mathcal{F}^{w \xi}, \mathcal{F}^{w \eta}\right)=\mathbf{B}^{b f}+\mathbf{B}^{s f}+\mathbf{B}^{w b}=\mathbf{B}^{b f}+\mathbf{B}^{s f}+\mathbf{B}^{w c a p}+\mathbf{B}^{b}+\mathbf{B}^{r}
$$

where $\mathbf{B}^{b f}$ and $\mathbf{B}^{s f}$ are accelerations due to bottom and surface streaming, respectively, while $\mathbf{B}^{w \boldsymbol{b}}$ denotes accelerations due to wave breaking. The latter is further decomposed to accelerations due to whitecapping ( $\left.\mathbf{B}^{\text {wcap }}\right)$, depth-limited wave breaking $\left(\mathbf{B}^{b}\right)$ and wave roller $\left(\mathbf{B}^{r}\right)$. It is important to point out that the contribution of bottom friction $\left(\epsilon^{b f}\right)$ is manifested in the form of bottom streaming, while the wave breaking induced acceleration $\left(\epsilon^{b}\right)$ is further divided into depth-limited breaking and roller contribution (see next few paragraphs). The model options used to activate these formulations within the COAWST modeling system are listed in Table 1.

\subsubsection{Bottom streaming $\left(\mathbf{B}^{b f}\right)$ term}

Interaction of waves with the sea bed leads to wave dissipation due to friction within the wave boundary layer. Three different bottom friction formulations are available in SWAN that are based on: (a) empirical formulations (JONSWAP) by Hasselmann et al. (1973); (b) the drag law model of Collins (1972); and (c) eddy viscosity model of Madsen et al. (1988). These formulations can be used to calculate $\epsilon^{b f}$ for a spectral wave field. In addition, the option for dissipation due to bottom drag using the parameterization presented by Reniers et al. (2004b) as in U10 has also been implemented (see Table 1). This option estimates $\epsilon^{b f}$ using:

$$
\epsilon^{b f}=\frac{1}{2 \sqrt{\pi}} \rho_{0} f_{w}\left|u_{o r b}^{w}\right|^{3} ;\left|u_{o r b}^{w}\right|=\frac{\sigma H_{*}}{2 \operatorname{sinhkD} D} ; f_{w}=1.39\left(\frac{\sigma z_{0}}{\left|u_{o r b}^{w}\right|}\right)^{0.52}
$$

where, $u_{o r b}^{w}$ is the bottom orbital velocity and $f_{w}$ is the wave friction factor (Soulsby, 1995).

Dissipation of wave energy in the wave boundary layer causes the instantaneous, oscillatory wave bottom orbital velocities ( $u$ ' and $w^{\prime}$ ) to be slightly in phase from quadrature causing a wave stress (bottom streaming) in the wave bottom boundary layer, along the direction of wave propagation (Longuet-Higgins, 1953; Phillips, 1977; Xu and Bowen, 1994; Lentz et al., 2008). This stress can be provided as a bottom stress or a body force. We have implemented two approaches to allow the effects of bottom streaming on the mean flows. First, following U10, the effect of bottom streaming in momentum balance is accounted for by using the wave dissipation due to bottom friction with an upward decaying vertical distribution.

$$
\mathbf{B}^{b f}=\frac{\epsilon^{b f}}{\rho_{0} \sigma} \mathbf{k} \cdot f^{b f}(z)
$$

where $f^{b f}(\mathrm{z})$ is a vertical distribution function given by: 


$$
f^{b f}(z)=\frac{\cosh \left(k_{w d}\left(\zeta^{c}-z\right)\right)}{\int_{-h}^{\zeta^{c}} \cosh \left(k_{w d}\left(\zeta^{c}-z\right)\right) d z}
$$

with $k_{w d}$ being a decay length which is a function of wave bottom boundary layer thickness ( $\left.\delta_{w}\right)$ and given by:

$$
k_{w d}=a_{w d} \delta_{w}
$$

where $a_{w d}$ is an empirical constant (=1 in here) and $\delta_{w}$ is a function of semi-orbital excursion ( $\left.A_{o r b}^{w}\right)$, Nikuradse roughness $\left(k_{n}\right)$ and bottom roughness length $\left(z_{0}\right)$.

$$
A_{o r b}^{w}=\left|\boldsymbol{u}_{\text {orb }}^{w}\right| / \sigma ; k_{n}=30 z_{0}
$$

As a second approach of bottom streaming, the method of Xu and Bowen (1994) is implemented (see Table 1) where:

$$
\mathbf{B}^{b f}=\frac{1}{\rho_{0}}\left\langle u^{\prime} w^{\prime}\right\rangle=\frac{H^{2} \sigma^{2} k}{8 \sinh ^{2} k h}\left[(-\beta z \cdot \sin \beta z+\beta z \cdot \cos \beta z-\cos \beta z) e^{-\beta z}+e^{-2 \beta z}\right]
$$

with $\beta=\sqrt{\sigma / 2 K_{m}}$, where $K_{m}$ is the eddy viscosity.

The first method is more suitable when the vertical resolution of the model is not high enough to resolve the wave bottom boundary layer while the second method is preferred for simulations that use high vertical resolution.

\subsubsection{Surface streaming $\left(\mathbf{B}^{\text {sf }}\right)$ term}

Similar to the concept of bottom streaming, at the surface of the water column the waveinduced stress develops a thin viscous boundary layer known as surface streaming (LonguetHiggins, 1953; Xu and Bowen, 1994 and Lentz et al., 2008). This contribution to nonconservative wave forcing is parameterized as (Xu and Bowen, 1994):

$$
\mathbf{B}^{s f}=\frac{K_{m} H^{2} \sigma}{2} \boldsymbol{k} \cdot k \cdot \operatorname{coth}(k h)
$$

and it is implemented as a surface boundary condition (see section 4). The effect of surface streaming can be interpreted in a similar manner as that of wind stress acting on the ocean surface (Weber et al., 2006). This effect may not be significant in a dynamic environment like the surf zone, but could be significant outside the surf zone as shown by Lentz et al. (2008).

\subsubsection{Wave Breaking $\left(\mathbf{B}^{w \boldsymbol{b}}\right)$ terms}

Non-conservative wave forcing due to wave breaking is traditionally defined only in a depth-averaged form (Longuet-Higgins, 1964; Smith, 2006). Newberger and Allen (2007a) implement the force due to depth induced breaking $\left(\mathbf{B}^{b}\right)$ as a surface stress, while Walstra et al. (2000), U10 and Kumar et al. (2011a) implement it as a surface intensified body force through the development of $a d-h o c$ vertical distribution functions. In the present work, we use a surface intensified distribution of $\mathbf{B}^{w c a p}, \mathbf{B}^{b}$ and $\mathbf{B}^{r}$ as in Kumar et al. (2011a).

\section{Whitecapping induced acceleration ( $\left.\boldsymbol{B}^{\text {wcap }}\right)$}


Whitecapping can occur in any water depth (van der Westhuysen et al., 2007; Jones and Monismith, 2008) as a response to wave steepening. Presently SWAN provides many different expressions for calculation of wave dissipation due to whitecapping (e.g., Rogers et al., 2003; van der Westhuysen et al., 2007). The associated acceleration is given as:

$$
\mathbf{B}^{\text {wcap }}=\frac{\epsilon^{\text {wcap }}}{\rho_{0} \sigma} \mathbf{k} \cdot f^{b}(z)
$$

where $\epsilon^{\text {wcap }}$ is the dissipation from SWAN, and $f^{b}(z)$ is the vertical distribution function such that:

$$
f^{b}(z)=\frac{\mathrm{FB}}{\int_{-h}^{\zeta^{c}} F B d z} ; F B=\cosh \left(\frac{2 \pi}{H}(z+h)\right)
$$

\section{Bathymetry induced breaking and acceleration $\left(\boldsymbol{B}^{\boldsymbol{b}}\right)$}

Depth limited wave breaking dissipation $\left(\epsilon^{b}\right)$ is computed in SWAN using a spectral version of the bore model based on Battjes and Janssen (1978), which depends on the ratio of wave height to water depth (see, Eldeberky and Battjes, 1996). Alternative empirical relationships for depth-induced breaking have been provided by Thornton and Guza (1983) and Church and Thornton (1993) and have been added as options (see Table 1). These formulations are:

$$
\begin{gathered}
\epsilon^{b}=\frac{3 \sqrt{\pi}}{16} \rho_{0} g \frac{B_{b}^{3} f_{p}}{\gamma_{b}^{4} D^{5}} H^{7} \\
\epsilon^{b}=\frac{3 \sqrt{\pi}}{16} \rho_{0} g \frac{B_{b}^{3} f_{p}}{D} H^{3}\left[1+\tanh \left\{8\left(\frac{H}{\gamma D}-1\right)\right\}\left[1-\left\{1+\left(\frac{H}{\gamma_{b} D}\right)^{2}\right\}^{-\frac{5}{2}}\right]\right.
\end{gathered}
$$

where, $H$ is the root mean square wave height; $f_{p}$ is the wave frequency; $g$ is the acceleration due to gravity; $B_{b}$ and $\gamma_{b}$ (the ratio of wave height to water depth) are empirical parameters.

The acceleration due to the depth-limited breaking dissipation is:

$$
\mathbf{B}^{b}=\frac{\left(1-\alpha^{r}\right) \epsilon^{b}}{\rho_{0} \sigma} \mathbf{k} \cdot f^{b}(z)
$$

where, $\alpha^{r}$ is the percentage of wave dissipation involved in creation of wave rollers (described in details below), and $f^{b}(z)$ is a vertical distribution function, where we have decided to use the same function as defined in Eqn. 30.

\section{Wave Rollers and Roller Acceleration $\left(\boldsymbol{B}^{\boldsymbol{r}}\right)$}

Within the surf zone the spatial distribution of wave dissipation is dominated by wave breaking that depends on bathymetry, but it is further modified due to the action of wave rollers. Wave rollers act as storage of dissipated wave energy, which is gradually transferred to the mean flow causing a lag in the transfer of momentum (Svendsen, 1984; Nairn et al., 1990). Warner et al. (2008a) and Haas and Warner (2009, hereinafter HW09) demonstrated the implementation and application into the ROMS model of a roller formulation based on Svendsen (1984). However, U10 presented a wave roller model which is similar to that of Reniers et al. (2004a) 
and Stive and DeVriend (1994). To provide additional capabilities, we also implemented this time dependent advective roller model into the COAWST system. The equations for evolution of wave rollers are similar to spectral wave evolution equation and can be represented as:

$$
\frac{\partial \mathcal{A}^{r}}{\partial t}+\nabla \cdot\left(\mathcal{A}^{r} \mathbf{c}\right)=\frac{\alpha_{r} \epsilon^{b}-\epsilon^{r}}{\sigma}
$$

where, $\mathcal{A}^{r}$ is the roller energy density; $\boldsymbol{c}$ is the phase speed of the primary wave, $\epsilon^{b}$ is the wave dissipation; $\epsilon^{r}$ is the roller dissipation rate; $\sigma$ is the wave frequency and $\alpha_{r}$ is an empirical parameter denoting the contribution of wave dissipation in creation of wave roller (see below). The roller energy density is related to roller energy by:

$$
\mathcal{A}^{r}=\frac{E^{r}}{\sigma}
$$

The phase speed of the primary wave is given by:

$$
\mathbf{c}=\overline{\mathbf{u}}+\sigma k^{-2} \mathbf{k}
$$

where, $\overline{\mathbf{u}}$ is the mean velocity, and $\mathrm{k}$ is the wave number. The roller dissipation rate is:

$$
\epsilon^{r}=\frac{g \cdot \sin \beta \cdot E^{r}}{c}
$$

where, $c$ is the phase velocity (Eqn. 4$)$ and $\sin \beta(=0.1)$ is an empirical constant (Reniers et al., 2004a).

As suggested by Tajima and Madsen (2006) and U10, the quantity $\alpha_{r}$ in Eqn. 34 can vary between 0 and 1 , providing a control on the amount of wave energy expended for the creation of wave rollers. This choice of $\alpha_{r}$ would be contingent upon wave breaking type (i.e., spilling, plunging, surging) which in turn depends on beach slope and type (Short, 1985).

The contribution of wave rollers in form of acceleration is given by:

$$
\mathbf{B}^{r}=\frac{\epsilon^{r}}{\rho_{0} \sigma} \mathbf{k} \cdot f^{b}(z)
$$

Combining Eqns. 29, 33 and 38 and after some re-organization the total acceleration contribution of the wave breaking term is written as:

$$
\mathbf{B}^{w b}=\frac{\left(1-\alpha^{r}\right) \epsilon^{b}+\epsilon^{r}+\epsilon^{w c a p}}{\rho_{0} \sigma} \mathbf{k} \cdot f^{b}(z)
$$

\subsection{Mass flux due to wave rollers}

The continuity equation (Eqn. 10) accommodates the mass flux due to Eulerian and Stokes transport. Wave rollers also contribute to associated mass flux increasing the total Stokes transport (Svendsen, 1984; Reniers et al., 2004a). The roller Stokes transport is given by:

$$
\mathbf{U}^{r}=\frac{E^{r}}{\rho_{0} \sigma} \boldsymbol{k}=\frac{\mathcal{A}^{r}}{\rho_{0}} \boldsymbol{k}
$$

and the total Stokes and roller transport becomes:

$$
\mathbf{U}^{S t}=\frac{\left(E+E^{r}\right)}{\rho_{0} \sigma} \boldsymbol{k}=\frac{\left(\mathcal{A}+\mathcal{A}^{r}\right)}{\rho_{0}} \boldsymbol{k}
$$




\begin{tabular}{|c|c|c|c|}
\hline \multicolumn{4}{|c|}{ VF options in the COAWST Modeling system. } \\
\hline Process & Switch Name & Description & References \\
\hline \multirow{3}{*}{$\begin{array}{c}\text { Wave } \\
\text { Dissipation }\end{array}$} & WDISS_WAVEMOD & Wave dissipation from wave model (SWAN) & SWAN manual \\
\hline & WDISS_THORGUZA & Wave-dissipation using Eq. 31 & $\begin{array}{l}\text { Thornton and } \\
\text { Guza (1986) }\end{array}$ \\
\hline & WDISS_CHURTHOR & Wave-dissipation using Eq. 32 & $\begin{array}{l}\text { Church and } \\
\text { Thornton } \\
\text { (1993) }\end{array}$ \\
\hline \multirow{2}{*}{ Roller Model } & ROLLER_RENIERS & $\begin{array}{l}\text { Solve the roller evolution equation (Eqn. } 34- \\
37 \text { ) to calculate roller dissipation. } \alpha_{r} \text { value is } \\
\text { provided by as an input parameter }\end{array}$ & $\begin{array}{l}\text { Reniers et al. } \\
\qquad(2004) ; \\
\text { Uchiyama et al. } \\
\text { (2010) }\end{array}$ \\
\hline & ROLLER_SVENDSEN & $\begin{array}{l}\text { Calculate roller area and roller energy using } \\
\text { Svendsen (1984) formulations }\end{array}$ & $\begin{array}{l}\text { Svendsen } \\
\quad(1984) \\
\text { Warner et al. } \\
\quad(2008)\end{array}$ \\
\hline \multirow{3}{*}{$\begin{array}{l}\text { Momentum } \\
\text { transfer due } \\
\text { to non- } \\
\text { conservative } \\
\text { forces }\end{array}$} & WEC_BREAKING & $\begin{array}{c}\text { Momentum contribution due to wave } \\
\text { breaking (Eqn. 33) }\end{array}$ & This paper \\
\hline & WEC_ROLLER & $\begin{array}{l}\text { Momentum contribution due to rollers (Eqn. } \\
\text { 38) }\end{array}$ & This paper \\
\hline & WEC_WCAP & $\begin{array}{l}\text { Momentum contribution due to whitecapping } \\
\text { (Eqn. 29) }\end{array}$ & This paper \\
\hline \multirow{3}{*}{ Streaming } & BOTTOM_STREAMING_YU & $\begin{array}{l}\text { Calculate wave dissipation due to bottom } \\
\text { friction (Eqn. 22) and the contribution to } \\
\text { momentum balance (Eqn. 23-26) }\end{array}$ & $\begin{array}{l}\text { Uchiyama et al. } \\
\qquad(2010)\end{array}$ \\
\hline & $\begin{array}{c}\text { BOTTOM_STREAMING_XU } \\
\text { _BOWEN }\end{array}$ & $\begin{array}{l}\text { Calculate contribution of bottom streaming to } \\
\text { momentum balance using Eqn. } 27\end{array}$ & $\begin{array}{l}\mathrm{Xu} \text { and Bowen } \\
\text { (1994) }\end{array}$ \\
\hline & SURFACE_STREAMING & $\begin{array}{l}\text { Calculate surface streaming contribution to } \\
\text { momentum balance using Eqn. } 28\end{array}$ & $\begin{array}{c}\text { Xu and Bowen } \\
\text { (1994) }\end{array}$ \\
\hline $\begin{array}{l}\text { Wave induced } \\
\text { mixing }\end{array}$ & TKE_WAVEDISS & $\begin{array}{l}\text { Compute TKE contribution due to wave } \\
\text { breaking using Eqn. 47, which is } \\
\text { implemented as a surface boundary condition } \\
\text { (Eqn. } 44,45 \text { ) to solve GLS model }\end{array}$ & $\begin{array}{l}\text { Feddersen and } \\
\text { Trowbridge } \\
\quad(2005)\end{array}$ \\
\hline
\end{tabular}

Table 1. COAWST options available for the computation of non-conservative wave forces (for details see Sec. 3.2). the effect of wave rollers is usually limited to the surface, a surface intensified distribution (e.g., HW09) may be more suitable. Simulations conducted using Stokes vs. surface intensified distribution provide similar results, so in the present implementation we use a Stokes velocity type distribution. 
Wave breaking induced dissipation leads to mixing of momentum in the water column (Agrawal et al., 1992). In surf zone this enhanced mixing can also be responsible for sediment resuspension in the water column (Voulgaris and Collins, 2000). The vertical scale of this mixing can be empirically related to the wave height (Rapp and Melville, 1990), which for shallow waters is usually of the same order as the water depth. This leads to a region in the water column of overlapped mixing due to wave breaking and turbulence from the bottom layer (Feddersen and Trowbridge, 2005).

Following Umlauf and Burchard (2003) as implemented in Warner et al. (2005), a generalized expression for transport of turbulent kinetic energy $(k)$ and generic length scale $(\psi)$ can be written as:

$$
\begin{gathered}
\frac{\partial k}{\partial t}+\boldsymbol{u} \cdot \nabla k=\frac{\partial}{\partial z}\left(\frac{K_{M}}{\sigma_{k}} \cdot \frac{\partial k}{\partial z}\right)+P+B-\varepsilon \\
\frac{\partial \psi}{\partial t}+\boldsymbol{u} \cdot \nabla \psi=\frac{\partial}{\partial z}\left(\frac{K_{M}}{\sigma_{\psi}} \cdot \frac{\partial k}{\partial z}\right)+\frac{\psi}{k} \cdot\left(c_{1} \cdot P+c_{3} \cdot B-c_{2} \cdot \varepsilon \cdot F_{\text {wall }}\right)
\end{gathered}
$$

where $P$ and $B$ are the shear and buoyancy production, respectively, $\sigma_{k}$ and $\sigma_{\psi}$ are turbulence Schmidt numbers for $k$ and $\psi$, respectively, and $F_{\text {wall }}$ is a wall function $c_{1}, c_{2}$ and $c_{3}$ are coefficients defined in detail in Warner et al. (2005). The generic length scale $(\psi)$ is defined as:

$$
\psi=\left(c_{\mu}^{0}\right)^{p} \cdot k^{m} \cdot l^{n}
$$

where, $c_{\mu}^{0}$ is a numerical constant; $m, n$ and $p$ are specified to relate $\psi$ to a turbulent quantity.

The turbulence due to injection of surface flux of TKE is given as surface boundary conditions (Craig and Banner, 1994; Feddersen and Trowbridge, 2005):

$$
\left.\frac{K_{M}}{\sigma_{k}} \frac{\partial k}{\partial z}\right|_{\zeta^{c}}=\overline{\epsilon_{w}}
$$

where $\overline{\epsilon_{w}}$ is the downward TKE flux due to breaking waves. The surface boundary condition for $\psi$ due to wave breaking is (Carniel et al., 2009):

$$
\begin{gathered}
\left.\frac{K_{M}}{\sigma_{\psi}} \frac{\partial k}{\partial z}\right|_{\zeta^{c}}=-\frac{\sigma_{k}}{\sigma_{\psi}} \cdot\left(c_{\mu}^{0}\right)^{p} \cdot m \cdot k^{m-1} \cdot\left(\kappa \cdot\left(z_{0}-z\right)\right)^{n} \cdot \mathscr{Y}- \\
\frac{v_{t}}{\sigma_{\psi}} \cdot\left(c_{\mu}^{0}\right)^{p} \cdot n \cdot k^{m} \cdot \kappa^{n} \cdot\left(z_{0}-z\right)^{n-1}
\end{gathered}
$$

In deep waters, $\mathscr{Y}=c_{w}\left(u_{s}^{*}\right)^{3}$, where $u_{s}^{*}$ is the friction velocity; and $c_{w}$ is a parameter that depends on the sea state, with a typical value of $c_{w}=100$ (Carniel et al., 2009). In the surf zone, $\mathscr{Y}=\overline{\epsilon_{w}} \cdot z_{0}$ is the surface roughness or the surface mixing length. For breaking wave conditions, the surface roughness is provided using the closure model of Stacey (1999):

$$
z_{0}=\alpha_{w} \cdot H
$$

where $\alpha_{w}=0.5$.

In the surf zone, part of the wave dissipation contributes to the flux of momentum (i.e., $\left(1-\alpha^{r}\right) \epsilon^{b}+\epsilon^{\text {wcap }}$, while the remaining amount (i.e., $\left.\alpha^{r} \epsilon^{b}\right)$ is expended for the creation of wave 
rollers. Furthermore, part of the wave and roller dissipation $\left(\epsilon^{r}\right)$ also contributes to turbulence mixing within the surf zone. Feddersen and Trowbridge (2005) assume that $25 \%$ of wave energy dissipation goes into the water column as TKE, while Jones and Monismith (2008) use a value of $6 \%$.

In the present work the contribution of wave dissipation as surface flux of TKE is expressed through an empirical coefficient $\mathrm{c}_{\epsilon w}$ which can be manually adjusted (see section 4.2.3) based on data availability. The surface flux of TKE is therefore:

$$
\overline{\epsilon_{w}}=\mathrm{c}_{\epsilon w}\left[\left(1-\alpha^{r}\right) \epsilon^{b}+\epsilon^{r}+\epsilon^{w c a p}\right]
$$

In order to conserve the total contribution to momentum balance due to wave dissipation, the amount of wave dissipation introduced as surface flux of TKE is subtracted from Eqn. 39.

\section{Model Simulations}

The modeling system with the VF formalism described above is applied to idealized and realistic surf zone and inner shelf environments to study the spatial variation and vertical structure of cross-shore and longshore flows. Four simulations are presented in detail, provided as standard test cases. The first two cases consist of creation of alongshore currents and undertow due to oblique incidence of spectral waves on a planar and a natural, barred beach assuming alongshore uniformity. The third case introduces three-dimensionality in the domain and flow development as it simulates a nearshore barred morphology interrupted by rip channels. The fourth case is designed to demonstrate the applicability of the model for inner shelf applications and simulates wave-induced cross-shore flows in the inner shelf. For all cases, an orthogonal coordinate system is defined so that $\mathrm{x}$ and $\mathrm{y}$ represent the cross-shore and longshore directions, respectively with positive $\mathrm{x}$ towards the open ocean. Correspondingly, positive cross-shore velocity values indicate offshore directed flow.

\subsection{Test Case 1: Obliquely incident waves on a planar beach}

The effect of VF formalism is examined through simulations for obliquely incident waves on a planar beach. This case has been previously discussed by HW09 and Kumar et al. (2011a) using depth dependent radiation stress formulations based on Mellor (2003) and Mellor (2008), respectively, and by U10 using a VF based model. In the simulations presented here, we use our implementation of the VF formulations which utilizes a different vertical distribution of wave dissipation and turbulence closure scheme than U10 (Eqns. 30 and 42).

The model domain has a cross-shore (x) width of $1,180 \mathrm{~m}$ and an alongshore $(\mathrm{y})$ length of $140 \mathrm{~m}$, with a $20 \mathrm{~m}$ grid resolution. The resting water depth varies from $12 \mathrm{~m}$ at the offshore boundary to $0 \mathrm{~m}$ at the shoreline. The vertical domain consists of 30 equally distributed vertical layers. The boundary conditions are periodic in the alongshore (i.e., north and south boundaries) and closed at the shoreline. At the offshore side we use Flather radiation condition (Flather, 1976) for free surface and Neumann boundary conditions for barotropic and baroclinic velocities (including boundary condition for Stokes velocities). The effect of earth rotation is not included. The bottom stress has been formulated using a quadratic bottom drag with a $\mathrm{c}_{\mathrm{d}}$ value of 0.0015 . The turbulence closure scheme is Generic Length Scale (GLS, $k-\varepsilon$ ) as described in Warner et al. (2005). Wave forcing is provided by SWAN, which propagates an offshore JONSWAP wave spectrum with a root mean square wave height $(H)$ of $1.4 \mathrm{~m}$, a peak period of $10 \mathrm{~s}$ and a $10^{\circ}$ 
angle of incidence. The barotropic and baroclinic time steps used are 0.16 and $5 \mathrm{~s}$, respectively. Effects of wave rollers, wave breaking induced mixing and bottom streaming are not included for this simulation, which is consistent with Kumar et al. (2011a), HW09 and U10.

Uchiyama et al. (2009) showed that in the presence of wave and current fluctuations, the mean continuity balance at steady state can be integrated in the cross-shore direction to yield a balance between barotropic Eulerian mean and Stokes velocities (i.e., $\bar{u}=-\overline{u^{S t}}$ ). This information along with the wave parameters and dissipation due to wave breaking $\left(\epsilon^{b}\right)$ can be used to solve for sea-surface elevation and barotropic longshore velocity using the following approximate equations:

$$
\begin{gathered}
\frac{\partial \zeta^{c}}{\partial x}=-\rho g h\left[\frac{\partial S_{x x}}{\partial x}\right]-\rho c_{d}|\boldsymbol{V}| \bar{u} \\
\rho c_{d}|\boldsymbol{V}| \bar{v}=\frac{\partial S_{x y}}{\partial x}=\frac{\epsilon_{b} k_{y}}{\sigma}
\end{gathered}
$$

where $|\boldsymbol{V}|$ is magnitude of the barotropic velocity vector, while $S_{x x}$ and $S_{x y}$ are onshore and longshore components of onshore radiation stress.

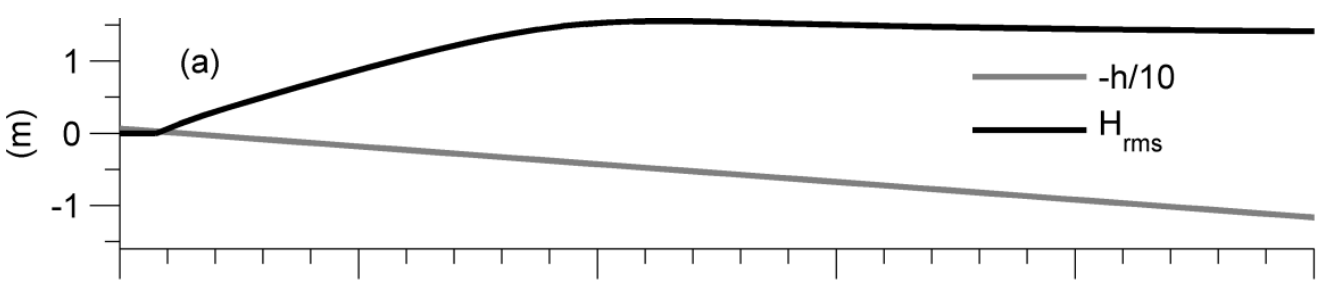

(b)

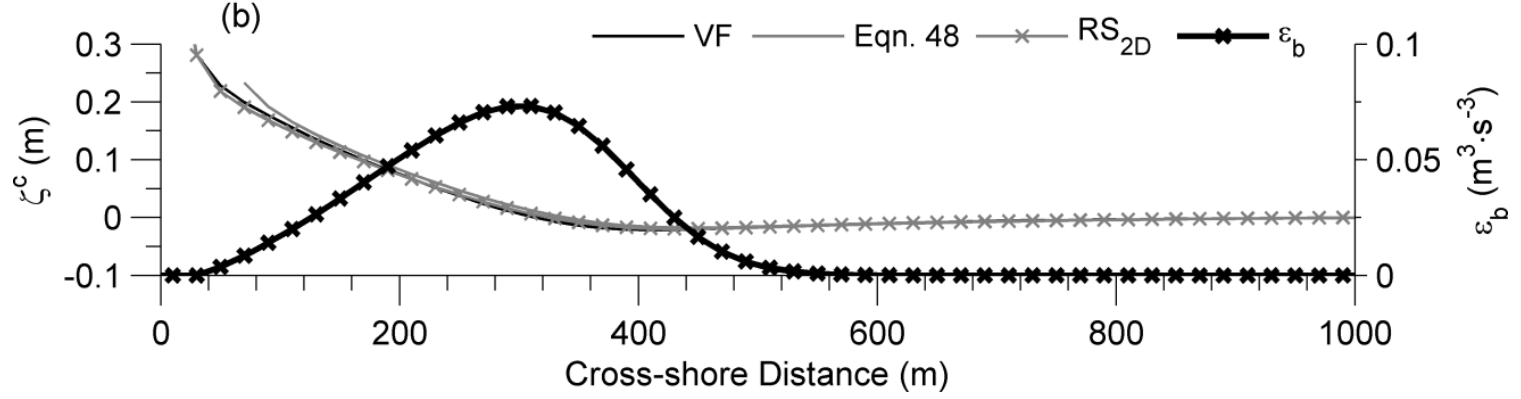

Figure 1. Obliquely incident waves on a planar beach simulated using the VF, the $\mathrm{RS}_{2 \mathrm{D}}$ model and analytical solution (see Eqn. 48). Cross-shore distribution of (a) root-mean-square wave height $(H)$ and water depth $(h)$; (b) sea surface elevation, $\zeta^{c}$ and depth-induced wave dissipation $\left(\varepsilon_{b}\right)$.

The results from the VF model simulation are compared to the analytical solutions of $\zeta^{c}$ and $\bar{v}$ obtained using Eqn. 48, with a $\mathrm{c}_{\mathrm{d}}$ value identical to that used in the numerical simulation, as well as to results from the depth-averaged, Lagrangian, radiation stress based model presented in Warner et al. (2008a, hereafter referred to as $\mathrm{RS}_{2 \mathrm{D}}$ ). In the latter model the wave forcing was provided as depth-averaged radiation stress (i.e., similar to Longuet-Higgins, 1970a and b).

\subsubsection{Wave parameters and sea-surface elevation}


Figures 1a and $\mathrm{b}$ show the wave height, depth-induced dissipation and sea surface elevation. Wave shoaling occurs in the region 500 to $1,000 \mathrm{~m}$; inshore of this region the waves start breaking in the depth-limited environment (Fig. 1a). Depth-induced dissipation $\left(\varepsilon_{b}\right.$, Fig. 1b) remains zero during wave shoaling. Inshore of $\mathrm{x}=500 \mathrm{~m}, \varepsilon_{b}$ increases monotonically to a maximum value of $0.07 \mathrm{~m}^{3} \mathrm{~s}^{-3}$ at $\mathrm{x}=300 \mathrm{~m}$, and then decreases gradually to zero at the shoreline. This depth-induced wave dissipation is the wave forcing which contributes to the momentum flux (Eqns. 33 and 12), leading to creation of longshore currents. Estimates of $\zeta^{c}$ from VF, $\mathrm{RS}_{2 \mathrm{D}}$ and the analytical solution (Eqn. 48) are in close agreement as shown in Figure 1b, with a slight difference at the coastline most likely due to lateral mixing or friction. Outside the surf zone, in the wave shoaling region, the mean sea level decreases (wave set-down), while within the surf zone, the mean sea level increases (wave set-up) as shown in Figure 1b.

\subsubsection{Nearshore flows}

Vertical variability of Eulerian mean and Stokes velocities from the VF simulation are shown in Figure 2. Inside the surf zone $(x<500$; Fig. 2a) the Eulerian mean cross-shore flow is inshore near the surface and offshore directed close to the sea bed. This vertical segregation of the cross-shore flow creates a circulation cell within the surf zone with downward and upward directed vertical velocities (see Fig. 2c), consistent with field observations of cross-shore velocity profiles for barred (Garcez-Faria et al., 2000), planar (Ting and Kirby, 1994) and laboratory (Roelvink and Reniers, 1994) beaches. Outside the surf zone the velocity is weakly offshore throughout the entire water column. These results are also consistent with U10, regardless of the differences in turbulence closure schemes and vertical distribution of wave dissipation. Depth-averaging the cross-shore Eulerian mean velocities shown in Figure 2a, we obtain velocities (Fig. 2g) that are equal in magnitude and opposite in sign to the depth-averaged Stokes velocity. This balance is indicative of a steady state solution achieved by the model and mass flux conservation.

The longshore velocity (Fig. 2b) attains its maximum value of approximately $-1 \mathrm{~ms}^{-1}$ at $\mathrm{x}=250 \mathrm{~m}$ and decreases to zero at the coastline and towards offshore. Vertically, the velocity shows maximum value at the surface and slightly lower values near the sea bed. Depth averaging these velocities, we find that the maximum alongshore velocity from the VF simulation is further inshore in comparison to the analytical solution, which shows a maximum value at $x=300 \mathrm{~m}$, at the same location as the maximum $\epsilon_{b}$ (Fig. 1b). This difference is mainly due to the inclusion of vertical viscous mixing, horizontal advection and VF leading to spreading and distribution of the momentum flux in the surf zone, something not included in the simplified analytical solution of Eqn. 48. Comparison to results obtained by $\mathrm{RS}_{2 \mathrm{D}}$ simulations are discussed separately in Section 5 .

The cross-shore Stokes velocity (Fig. 2d) is one and two orders higher than the longshore (Fig. 2e) and vertical Stokes velocity (Fig. 2f), respectively. Close to the sea surface, cross-shore velocity varies from zero at the offshore boundary to a maximum value of $\sim-0.15 \mathrm{~ms}^{-1}$ at the location of maximum wave breaking (i.e., $x=300 \mathrm{~m}$ ), decreasing with increasing water depth. Further inshore of this position, the cross-shore velocity reduces to zero. Longshore velocity is weaker in strength, but shows a distribution similar to that of the cross-shore Stokes velocity. Since the vertical Stokes velocity is calculated as divergence of horizontal mass flux (Eqn. 2), at the location of maximum breaking, the vertical Stokes velocity is zero. Inshore of this point, the 
velocity is positive with a maximum value at the surface, decreasing with increasing water depth. Offshore of the break point, the velocity is negative and downwards directed, with a vertical structure similar to other Stokes velocity components. The vertical Stokes velocity has similar magnitude $\left( \pm 0.005 \mathrm{~ms}^{-1}\right)$ but opposite sign to the vertical Eulerian mean flows (Fig. 2c).
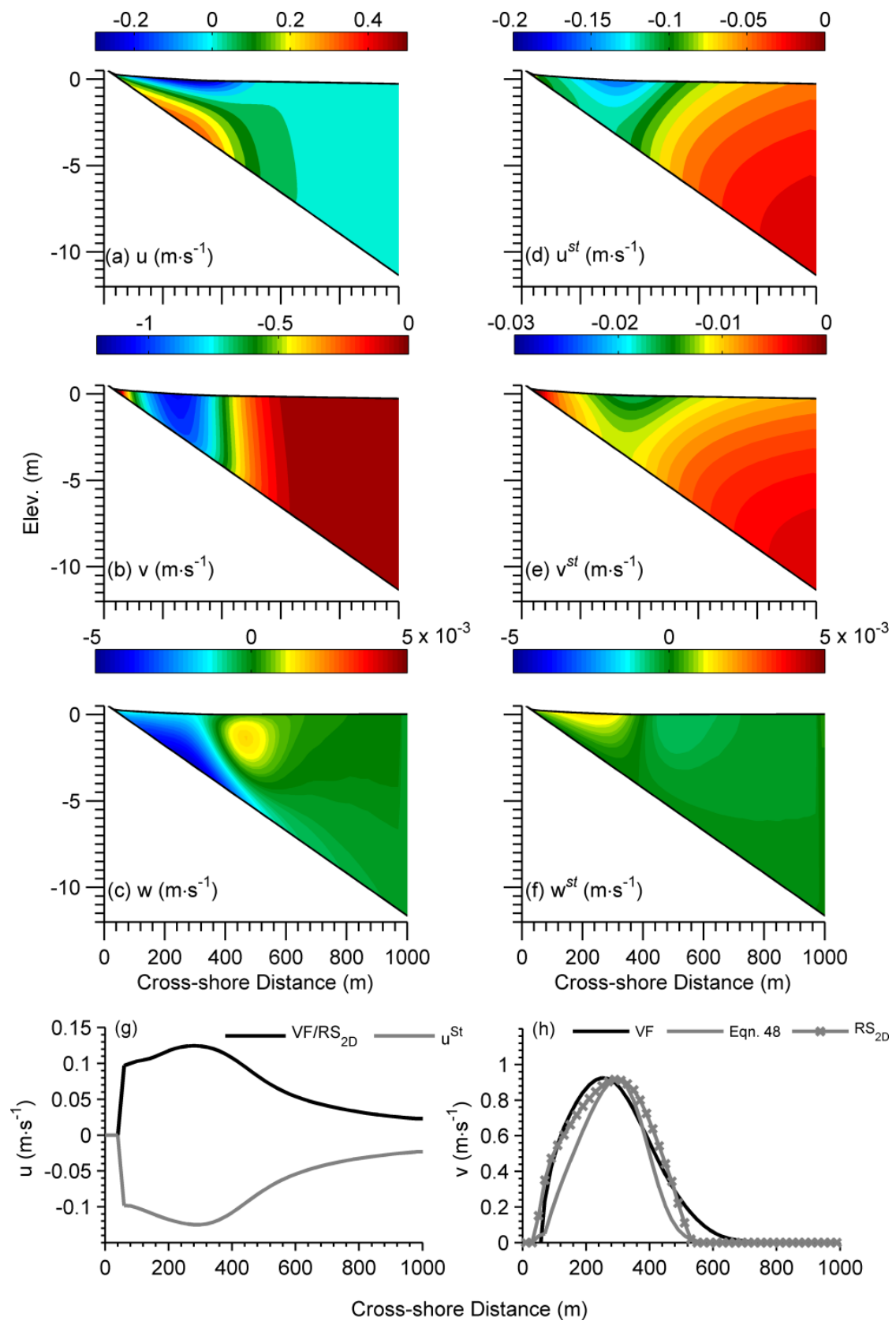

Cross-shore Distance $(\mathrm{m})$

Figure 2. Cross-shore sections of Eulerian ( $a, b$ and $c$ ) and Stokes (d, e and f) velocities from the VF model. (a) cross-shore $(u)$; (b) longshore $(v)$; and (c) vertical $(w)$ Eulerian velocities. (d) cross-shore $\left(u^{s t}\right)$; (e) alongshore $\left(v^{s t}\right)$; (f) vertical $\left(w^{s t}\right)$ Stokes velocities; Cross-shore distribution of (g) depth-averaged, cross-shore Eulerian velocity $(\bar{u})$ and Stokes velocity $\left(\overline{u^{S t}}\right)$; and (h) depth-averaged, alongshore $(\bar{v})$ 
Eulerian velocity for obliquely incident waves on a planar beach simulated using the VF, the $\mathrm{RS}_{2 \mathrm{D}}$ model and analytical solution (see Eqn. 48).

\subsubsection{Three-dimensional momentum balance}

The relative contribution of the cross-shore (x) and longshore (y) momentum balance terms are described here, using the nomenclature as shown in Eqn. 11 and 12 corresponding to acceleration (ACC), horizontal and vertical advection (HA and VA), Coriolis force (COR), Stokes-Coriolis force (StCOR), pressure gradient (PG), horizontal VF (HVF), horizontal and vertical mixing (HM and VM), and breaking and roller acceleration (BA and RA). Though the contribution of vertical vortex force (K, Eqn. 1) can be analyzed separately as a part of the geopotential function (Eqn. 13), in this work we have added it to the HVF term, as its importance is negligible in all the cases discussed here.

In the cross-shore direction (Fig. 3), since earth rotation and roller efect were not considered, the RA, COR and StCOR terms are zero. The horizontal advection (HA, Fig. 3b), horizontal vortex force (HVF, Fig. 3c) and vertical advection (VA, Fig. 3f) terms are negligible. The balance is mainly between three terms: BA, VM and PG. Within the surf zone $(x<350 \mathrm{~m})$, the wave breaking acceleration (BA, Fig. 3a) term is the largest with a high value at the sea surface, sharply decreasing to a negligible value below $1 \mathrm{~m}$ under the surface. A significant portion of the BA contribution is balanced by a relatively strong vertical mixing (VM, Fig. 3e) which is enhanced close to surface layer. At water depths where BA becomes negligible, the VM changes sign and becomes negative. At the location where waves start breaking (i.e., $350 \mathrm{~m}<\mathrm{x}<$ $500 \mathrm{~m}$ ), the contribution of pressure gradient (PG, Fig. 3d) is negligible, but increases toward the shoreline, with a vertically uniform distribution. Close to the sea surface, both PG and VM terms add to balance the BA contribution while further below the balance is mainly between PG and $\mathrm{VM}$, with the latter term also becoming vertically uniform. It is important to note that comparing the present momentum balance to that obtained from simulations using models based on depth varying radiation stress (e.g., Kumar et al., 2011a) and quasi-3D models such as SHORECIRC (e.g., HW09) we find that in the VF formulation, the VM term is responsible for vertically redistributing the BA and balancing PG. In the former two models the primary balance occurs between vertically uniform PG and almost vertically uniform radiation stress contribution.

The major terms in alongshore momentum balance are BA, HA, HVF, VM and VA, while PG is negligible. BA (Fig. 3g) is dominant only in the surface layer within the wave breaking zone where significant part of it is balanced by the VM term (Fig. 3k). Further below the sea surface (> $1 \mathrm{~m}$ ), VM changes sign from positive to negative, and when added to VA and HVF the sum balances HA (Figs. 3h, i, k and l). HA and VA terms (Figs. 3h and l) show opposite signs over the entire water column, which can be attributed to vertical segregation of cross-shore velocity (Fig. 2a) and change in the gradient, inshore and offshore of the location of maximum undertow. The HVF term (Fig. 3i) is zero at the location of maximum longshore velocity (as $\partial \mathrm{v} / \partial \xi$ is zero, see Eqn. 12), and has opposite signs on either side of this point. Overall, at locations inshore of the longshore flow maximum $(x<260 \mathrm{~m})$ the sum of BA, HA and HVF is balanced by the sum of VA and VM near the sea surface; close to the bed, the sum of HVF, VM and VA is balanced by HA. Similar balances also occur at locations further offshore $(\mathrm{x}>260 \mathrm{~m})$. 


\subsubsection{Balance of vertically-integrated three-dimensional momentum balance}

The vertically averaged cross-shore momentum balance terms (Fig. 4a) show a balance between PG and BA similar to that presented analytically by Bowen et al. (1968) for a planar beach. The contribution of the remaining terms (including HVF) is negligible. Vertical integration of the longshore momentum terms (Fig. 4b) shows a primary balance between the BStr (vertical integral of VM term, Eqn. 20) and the BA terms. A secondary balance occurs between the HVF and the HA terms. The horizontal vortex force (HVF) term is positive seaward of the location of maximum longshore current and becomes negative inshore that location, and the horizontal advection (HA) is of similar magnitude as the HVF but of opposite sign. This secondary balance suggests a balance between Stokes and anti-Stokes (Eulerian mean) flows; however, these terms do not cancel out completely due to differences in vertical structure of Stokes and Eulerian mean flows (see Sec 5).
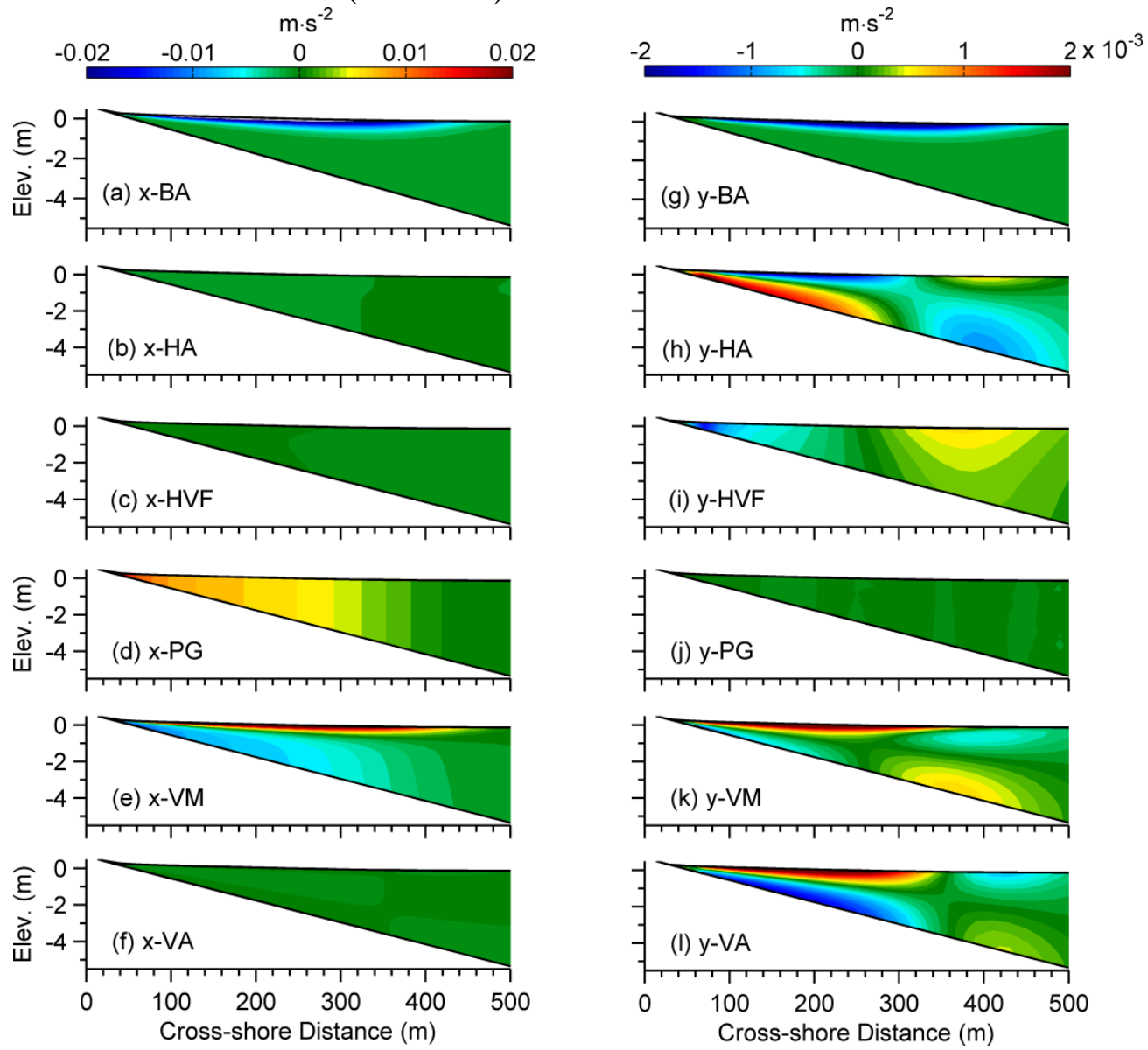

Figure 3. Vertical and horizontal cross-shore distribution of the various cross-shore (x) and longshore (y) momentum balance terms. Cross-shore terms: (a) x- breaking acceleration (x-BA) ; (b) x-horizontal advection (x-HA); (c) x-horizontal vortex force (x-HVF); (d) $x$ - pressure gradient (x-PG); (e) $x$ - vertical mixing $(\mathrm{x}-\mathrm{VM})$; and (f) $\mathrm{x}$-vertical advection $(\mathrm{x}-\mathrm{VA})$; Longshore terms: $(\mathrm{g}) \mathrm{y}$ - breaking acceleration (y-BA) ; (h) y-horizontal advection (y-HA); (i) y-horizontal vortex force (y-HVF); (j) y- pressure gradient (y-PG); (k) y-vertical mixing (y-VM); and (l) y-vertical advection (y-VA)term. 
In the present modeling framework, the terms contributing to the total pressure gradient force ( $\nabla \varphi$ from Eqn. $13=\boldsymbol{P}^{\text {tot }}$, i.e., gradient of dynamically relevant kinematic pressure), excluding the vertical vortex force $(\mathbf{K})$, can be decomposed into two terms that describe individual contributions from the Eulerian non-WEC $\left(\boldsymbol{P}^{c}\right)$ and WEC $\left(\boldsymbol{P}^{\text {wec }}\right)$ contributions. The latter can be further divided into a quasi-static response $\left(\boldsymbol{P}^{q s}\right)$, a Bernoulli head $\left(\boldsymbol{P}^{b \boldsymbol{h}}\right.$, see Eqn. 5) and a surface pressure boundary correction $\left(\boldsymbol{P}^{p c}\right)$ (see Table 2):

$$
\begin{aligned}
\boldsymbol{P}^{t o t} & =\boldsymbol{P}^{c}+\boldsymbol{P}^{w e c}=\left(\boldsymbol{P}^{c}\right)+\boldsymbol{P}^{q s}+\boldsymbol{P}^{b h}+\boldsymbol{P}^{p c} \\
& =-\nabla_{\perp}\left(g \zeta^{c}+\int_{-h}^{\zeta^{c}} \frac{g \rho}{\rho_{0}} d z\right)+g \nabla_{\perp} \hat{\zeta}+\left.\nabla_{\perp} \mathcal{K}\right|_{\zeta^{c}}+\left.g \nabla_{\perp} \mathcal{P}\right|_{\zeta^{c}}
\end{aligned}
$$

Table 2. List of the components which constitute the total pressure gradient force (Eqn. 49)

\begin{tabular}{|c|c|}
\hline $\begin{array}{c}\text { Individual } \\
\text { Terms }\end{array}$ & Description \\
\hline$P^{t o t}$ & Total pressure gradient force. Contribution of both WEC and non-WEC terms \\
\hline$P^{c}$ & Non-WEC current contribution \\
\hline$P^{\text {wec }}$ & WEC contribution $\left(P^{q s}+P^{b h}+P^{p c}\right)$ \\
\hline$P^{q s}$ & Quasi-static response (Eqn. 7) \\
\hline$P^{b h}$ & Bernoulli head (Eqn. 5) \\
\hline$P^{p c}$ & Surface pressure boundary correction (Eqn. 9) \\
\hline
\end{tabular}

Analysis of the individual components of pressure gradient force (PG, Fig. 4c) show that major contribution to $P^{\text {totx }}$ is from the non-WEC response of the system to wave breaking, (i.e., $\left.P^{c x}\right)$. Outside the surf zone, quasi-static response $\left(P^{q s}\right)$ and $P^{c x}$ balance each other which cause the wave set-down at this location. The terms corresponding to Bernoulli head and dynamic surface boundary correction are negligible for this planar beach case.

\subsection{Test Case 2: Obliquely incident waves on a natural, barred beach (DUCK' 94 Experiment)}

In this test case we simulated wave-induced currents for a natural, barred beach corresponding to the DUCK'94 experiment (Gallagher et al., 1998; Elgar et al., 1997; Feddersen et al., 1998). Simulations are compared to data collected on Oct $12^{\text {th }}, 1994$, when strong velocities were observed in the surf zone due to waves generated by winds associated with the passage of a low-pressure storm system (Garcez-Faria et al., 2000). During this period, both waves and winds were directed towards the southwest generating a longshore flow down-coast (i.e., towards southeast, see Garcez-Faria et al., 1998).

The measured bathymetry, shown in Figure 5a, originates with the shoreline at $\mathrm{x}=0$ and the nearshore bar located near $x=130 \mathrm{~m}$. The model domain is assumed alongshore uniform with a cross-shore (x) width of $780 \mathrm{~m}$ and a horizontal resolution of $2 \mathrm{~m}$. The water depth varies from $0 \mathrm{~m}$ at the shoreline to $7.26 \mathrm{~m}$ at the offshore boundary. A tidal elevation of $0.70 \mathrm{~m}$ was added to the water level and assumed constant over the simulation period (simulations with tidal variability did not show substantial changes in the model results). The vertical dimension is discretized with 32 equally distributed layers. The boundary conditions are periodic in the alongshore (i.e., north and south boundaries) and closed at the shoreline. At the offshore end we 
use Flather radiation condition (Flather, 1976) for free surface and Neumann boundary conditions for barotropic and baroclinic velocities (including boundary condition for Stokes velocities). Effect of earth rotation is not included. Bottom stress due to the combined action of waves and currents is estimated using a benthic boundary layer formulation (Madsen, 1994) as described in Warner et al. (2008). Weak horizontal momentum diffusion of the order $0.05 \mathrm{~m}^{2} . \mathrm{s}^{-1}$ is also applied to obtain smooth solutions. The turbulence closure scheme used is Generic Length Scale (GLS, $k-\varepsilon$ ). Wind stress forcing of 0.25 and $0.16 \mathrm{Nm}^{-2}$ is imposed in the cross-shore and longshore directions, respectively. Wave forcing is provided by SWAN, which propagates an offshore JONSWAP wave spectrum with a significant wave height of $2.3 \mathrm{~m}$, a peak period of $6 \mathrm{~s}$ and a $13^{\circ}$ angle of incidence. The model simulation is carried out for a period of 3 hours with a baroclinic and barotropic time stepping of 3.0 and $0.1 \mathrm{~s}$, respectively.
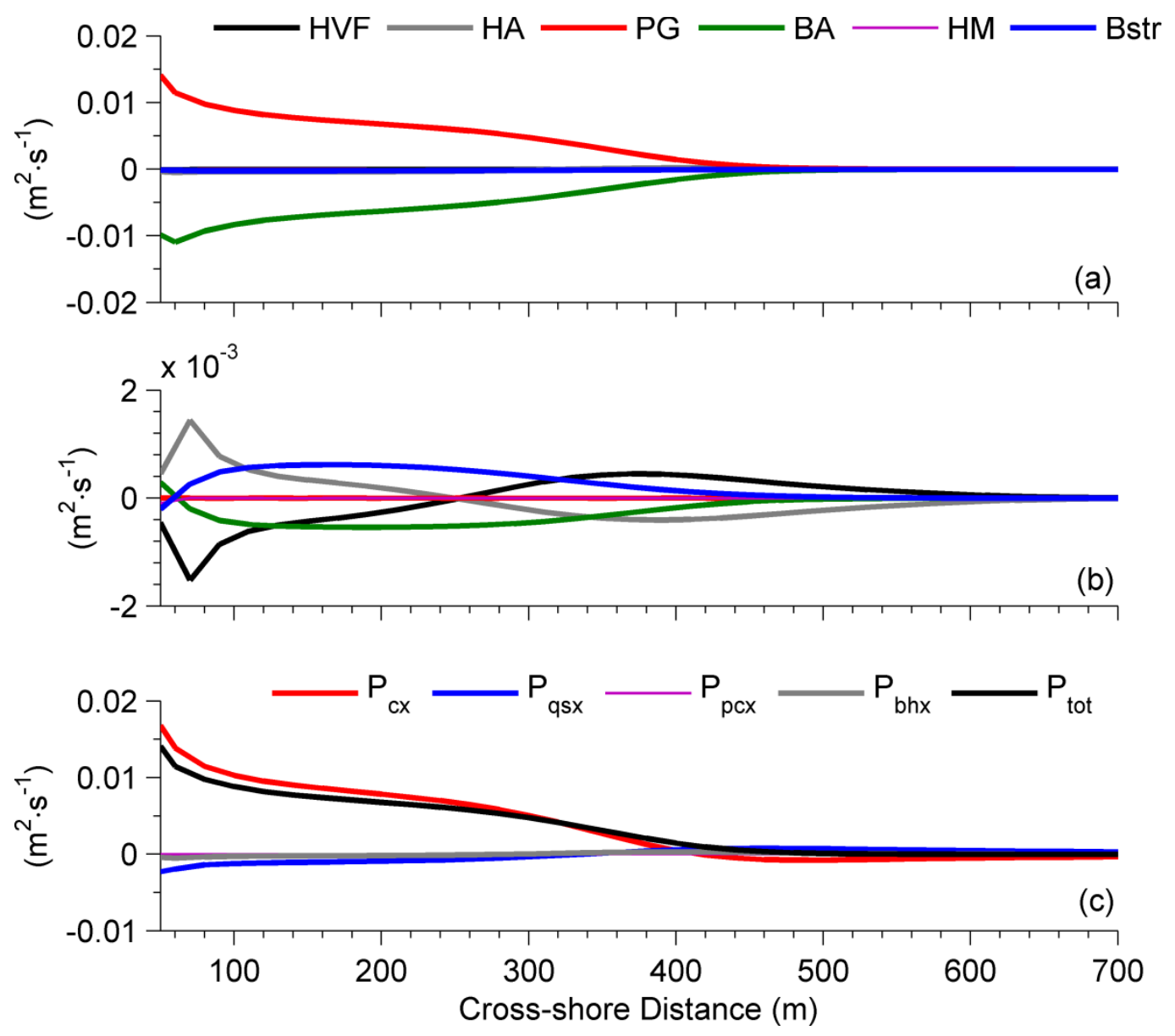

Figure 4. Cross-shore variation of depth-averaged (a) cross-shore; (b) longshore momentum balance terms; and (c) decomposed PGF terms in cross-shore as described in Eqn. 49.

Ten different simulations were carried out in order to identify the behavior of wave rollers and wave-induced mixing. The simulations are designated as Run \# (where \# is the simulation number) and the differences between individual Runs are listed in Table 3. Run 1 is conducted using the two-dimensional (x-y), depth-averaged, Lagrangian, radiation stress based model $\left(\mathrm{RS}_{2 \mathrm{D}}\right.$, i.e., no vertical distribution of wave forcing or flows, and the wave forcing is depth-averaged radiation stress contribution as in Longuet-Higgins, 1970a), while for Runs 2 to 
10 we use the vortex force formulation as described in this paper (VF). Run 2 does not include the effect of wave rollers and wave-induced mixing. Runs 3 to 6 do include the effect of wave rollers but each run assumes a different fraction (Eqn. 34) of depth-induced dissipation $\left(\epsilon_{b}\right)$ being used for roller generation. Finally, Runs 7 to 10 are used to distinguish the contribution of wave-induced mixing.

Table 3. Model configuration for different DUCK' 94 simulations. $\mathrm{RS}_{2 \mathrm{D}}$ refers to simulations conducted using depth-averaged, radiation stress based model, while simulations done using the VF formalism are referred to as VF. $\alpha_{r}$ is the coefficient which determines the percentage of wave breaking induced dissipation contributing to creation of wave rollers (Eqn. 34), while $c_{\varepsilon w}$ is the percentage of total dissipation going as turbulent kinetic energy (Eqn. 43 and 47).

\begin{tabular}{|c|c|c|c|c|c|}
\hline \multicolumn{6}{|c|}{ Description of Model Runs for DUCK' 94 Experiment } \\
\hline Run \# & $\begin{array}{c}\text { Model } \\
\text { Formulation }\end{array}$ & $\begin{array}{c}\text { Effect of } \\
\text { Wave Rollers }\end{array}$ & $\alpha_{r}$ & $\begin{array}{c}\text { Surface } \\
\text { TKE }\end{array}$ & $c_{\varepsilon w}$ \\
\hline 1 & $\mathrm{RS}_{2 \mathrm{D}}$ & OFF & - & - & - \\
\hline 2 & VF & OFF & 0 & OFF & 0 \\
\hline 3 & VF & ON & 0.25 & OFF & 0 \\
\hline 4 & VF & ON & 0.50 & OFF & 0 \\
\hline 5 & VF & ON & 0.75 & OFF & 0 \\
\hline 6 & VF & ON & 1.00 & OFF & 0 \\
\hline 7 & VF & ON & 0.5 & ON & 0.01 \\
\hline 8 & VF & ON & 0.5 & ON & 0.05 \\
\hline 9 & VF & ON & 1.0 & ON & 0.01 \\
\hline 10 & VF & ON & 1.0 & ON & 0.05 \\
\hline
\end{tabular}

\subsubsection{Wave parameters and sea-surface elevation}

In this section we first examine two runs: (a) Run 1 (radiation stress based, depthaveraged model with no rollers) and (b) Run 2 (baseline experiment using VF model without wave rollers and mixing), to compare the flow pattern simulated by a two and three-dimensional model.

Measured (Elgar et al., 1997) and simulated $H$ are in a close agreement throughout the profile (Fig. 5a), despite the fact that the wave solution does not account for the effect of currents (one-way coupling). Depth-limited wave breaking, as exhibited through the wave dissipation $\left(\varepsilon_{b}\right)$ parameter, takes place predominantly over the bar-crest and then a second time close to the shoreline (Fig. 5b). Over the bar-trough $(60 \mathrm{~m}<x<100 \mathrm{~m})$, the wave dissipation is negligible, as shown by the relatively stable wave height along this region (Fig. 5a).The overall trend of seasurface elevation $\left(\zeta^{c}\right)$ for both Runs 1 and 2 is a wave set-down outside and wave setup inside the surf zone (Fig. 5b). At the bar-crest and further shoreward, $\zeta^{c}$ from Run 1 shows a continuous increase, unlike Run 2, which suggests slight decrease at these locations due to dominant contribution of Bernoulli head (see section 5).

\subsubsection{Nearshore flows}


The cross-shore profiles of depth-averaged, cross-shore and longshore velocities from Runs 1 ( $\mathrm{RS}_{2 \mathrm{D}}$, no vertical flow distribution) and 2 (VF, no rollers/mixing, vertically averaged velocities) are shown in Figures 5c and d. Although the depth-averaged cross-shore velocities from Runs 1 and 2 are identical (Fig. 5c), the longshore velocities show significant difference both in terms of cross-shore variability and magnitude (Fig. 5d). Strongest longshore velocity from Run 1 occurs at the bar-crest and at locations close to the shoreline, which does not agree with the observations. On the other hand, maximum longshore current from Run 2 is at a location inshore of the bar-crest, and is in better agreement with measured velocities. This inshore shift of the maximum longshore current is due to vortex force and mixing due to shear, details of which are provided in section 4.2 .7 and 5.
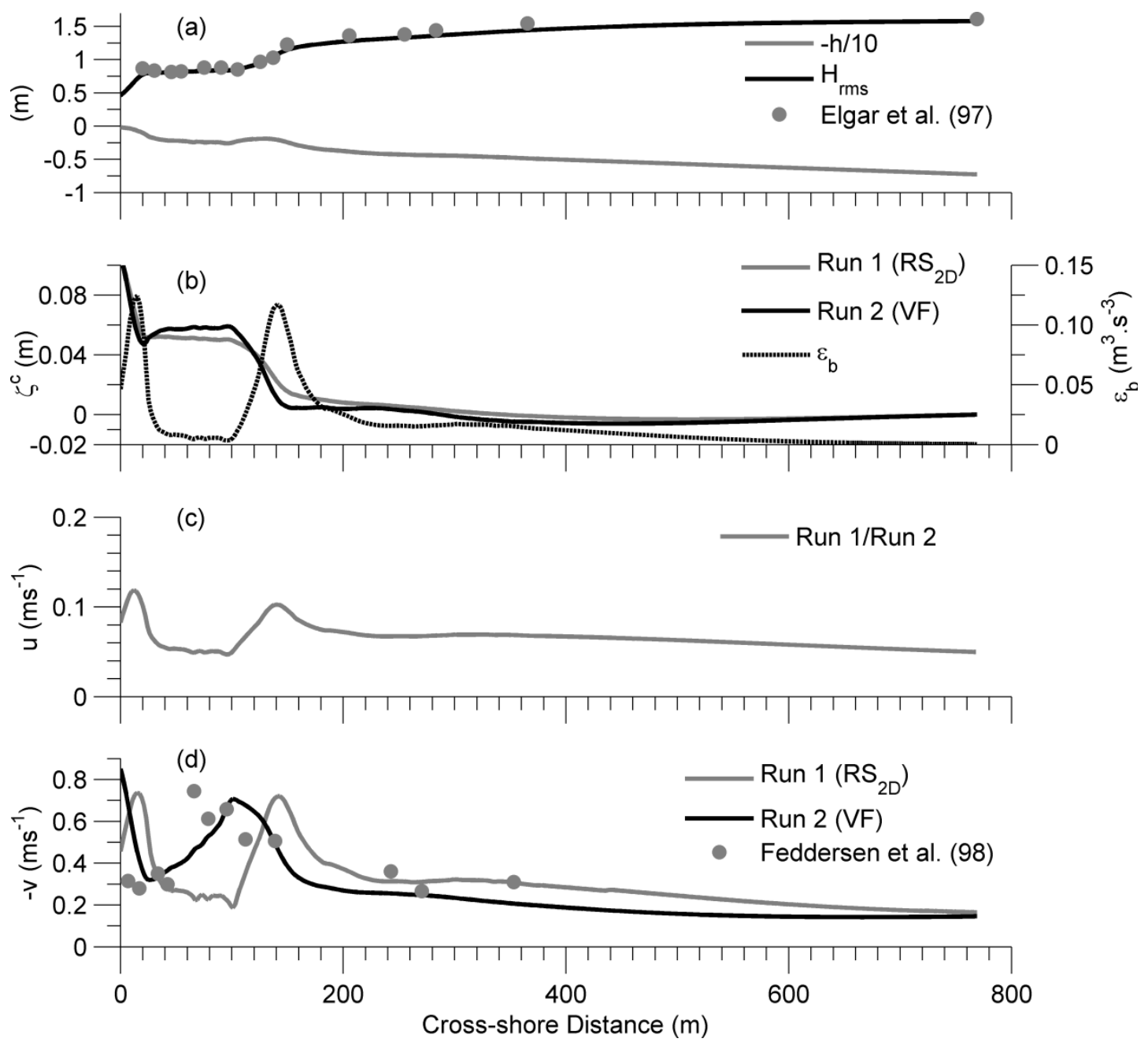

Figure 5. Obliquely incident waves on a barred beach simulated using the VF model (no roller model, i.e., Run 2) and the $\mathrm{RS}_{2 \mathrm{D}}$ (Run 1) model (see Table 3). Cross-shore distribution of: (a) root mean square wave height $\left(\mathrm{H}_{\text {rms }}\right)$ from SWAN (solid black line), observed wave height (from Elgar et al., 1997; grey circles) and water depth (h). (b) Sea surface elevation $\left(\zeta^{c}\right)$ and depth-induced wave dissipation $\left(\varepsilon_{\mathrm{b}}\right)$. (c) Depthaveraged, cross-shore Eulerian velocity, $(\bar{u})$. (d) Depth-averaged, longshore Eulerian velocity $(\bar{v})$ along with observed velocity (from Feddersen et al., 1998; grey circles). 


\section{Effect of wave roller}

Wave roller generation is controlled through the parameter $\alpha_{r}$, which defines the fraction of wave dissipation allowed to act as the source term in the roller evolution equation (Eqns. 33, 34 and 37). When $\alpha_{r}=0$, no wave rollers are included, while when $\alpha_{r}=1$ the total of the depthinduced dissipation $\left(\epsilon_{b}\right)$ is used as a source for the creation of wave rollers. The roller dissipation is calculated empirically (Eqn. 37) which contributes to roller acceleration in the momentum balance along with breaking acceleration (Eqn. 39). Five simulations with no wave-induced mixing and $\alpha_{r}$ values of $0.25,0.5,0.75$ and 1 (Runs 2 to 6 , respectively, see Table 3 ) were carried out and the total dissipation $\left(=\left(1-\alpha_{r}\right) \epsilon_{b}+\epsilon_{r}\right)$ for each run is shown in Figure 6a. When $\alpha_{r}=0$, maximum depth-induced dissipation is observed at the bar-crest and close to the shoreline. As the value of $\alpha_{r}$ increases, the contribution of breaking dissipation decreases and the contribution of roller dissipation increases. The advection of wave rollers with a speed equal to the phase speed of the surface gravity waves leads to an onshore movement of the total dissipation peak (Fig. 6a). For $\alpha_{r}=1$, the total dissipation decreases at the bar-crest and close to the shoreline, and increases in the bar-trough region, providing a wider distribution of the energy lost by breaking waves. Physically this mechanism modifies the setup in the transition zone (Nairn et al., 1990), creates a delay in the transfer of energy from wave breaking to the mean flow (Reniers and Battjes, 1997; Ruessink et al., 2001) and accounts for the associated mass flux in the direction of wave propagation (Svendsen, 1984). In the next three sub-sections we describe the physical impact of wave rollers in modifying the cross-shore profile of barotropic flows, cross-shore profile and vertical structure of cross-shore and longshore current, vertical profile of eddy viscosity and turbulent kinetic energy. The simulated flows are also compared to field measurements of cross-shore and longshore velocities.

In absence of any other forcing mechanism and under steady state conditions, the vertically averaged Stokes flow is balanced by an opposing Eulerian mean flow (Uchiyama et al., 2009). In absence of wave rollers (Fig. 6b) this flow is strongest at the location of wave breaking (i.e., bar-crest and at the shoreline). As the contribution of wave rollers increase, the rollers contribute an onshore directed mass flux, leading to a stronger return flow in the offshore direction (Fig. 6b). Changes in wave roller contribution also affect the cross-shore variation of the depth averaged longshore currents (see Fig 6c). When $\alpha_{1}=0$ (Run 2), the maximum longshore velocity is predicted just inshore of the bar-crest. Increasing the wave roller delays the transfer of energy from waves to mean flow, leading to a more uniform distribution of flow within the areas inshore and offshore of the bar-trough $(80-100 \mathrm{~m})$. When $\alpha_{l}=1$, relatively stronger longshore velocity is modeled inshore of the bar-trough.

Simulated profiles of cross-shore and longshore velocity from Runs 2, 4 and 6 (i.e., VF based model with $\alpha_{1}=0,0.5$ and 1 , respectively, see Table 3 ) are compared to observations (Garcez-Faria et al., 1998, 2000) at seven different cross-shore locations spanning the region between the bar-trough and crest (Figs. $7 \mathrm{a}$ and $\mathrm{b}$ ). The normalized root mean square (rms) errors (defined same way as in Newberger and Allen, 2007b) for each simulation and cross-shore location are listed in Table 4.

The observed cross-shore velocities (Fig. 7a) show a strong vertical shear at the bar-trough and bar-crest regions, creating a circulation pattern with inshore directed flows at the surface and offshore directed undertow close to the bed. Simulated velocity profiles from Runs 2, 4 and 6 (VF based model with $\alpha_{1}=0,0.5$ and 1 , respectively) show similar general pattern. When $\alpha_{1}=0$ (Run 2), the velocity shear is strongest over the bar-crest, while when $\alpha_{r}=1$ (Run 6) velocity 
shear increases at the bar-trough region (Fig. 7a). It is also shown that the undertow strength increases with an increased roller contribution due to additional return flows generated to compensate for the increased mass flux due to rollers. Overall, Run 6, a case where the entire wave dissipation is converted to wave rollers (i.e, $\alpha_{r}=1$ ), shows the best agreement with the measured cross-shore velocities as revealed by their least rms error values.
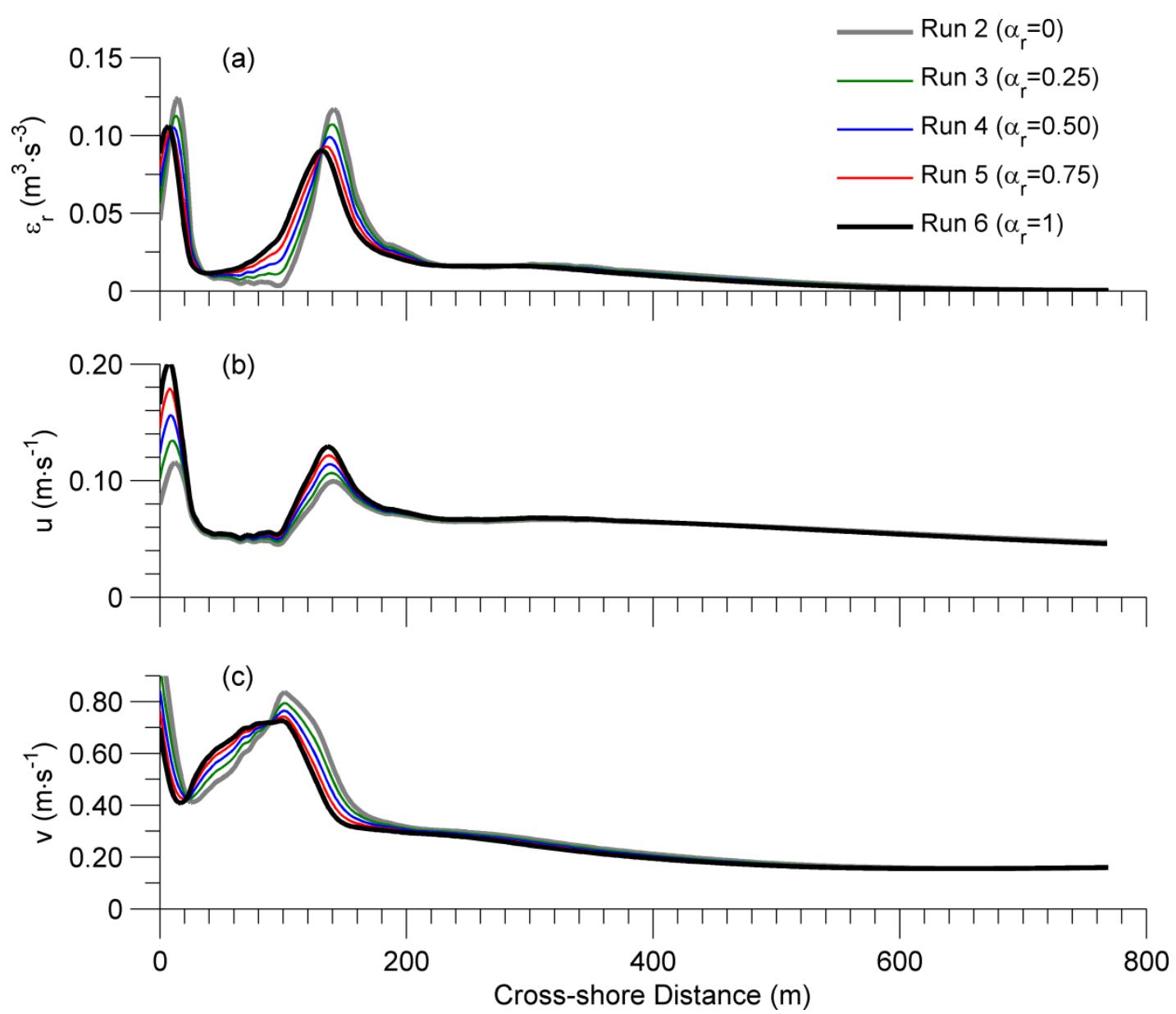

Figure 6. Cross-shore variability of (a) total dissipation (breaking + roller dissipation) and depth-averages of three-dimensional (b) cross-shore, $\bar{u}$ and (c) longshore velocity, $\bar{v}$ estimates, for different values of $\alpha_{r}$ (Runs 2-6, Table 3).

The measured longshore velocity is highest in the bar-trough region and gradually decreases on either side (Fig. 7b). When the roller effect is not considered (i.e., $\alpha_{l}=0$, Run 2), the longshore velocity maximum occurs in the region between the bar-trough and crest $(\mathrm{x} \sim 110 \mathrm{~m})$. As the roller contribution increases to $50 \%$ (i.e., $\alpha_{r}=0.5$, Run 4 ), this local maximum is shifted further inshore at $\mathrm{x}=100 \mathrm{~m}$ (Fig. $7 \mathrm{~b})$. When the total dissipation is used to generate wave rollers $\left(\alpha_{r}=1\right.$, Run 6), the longshore velocity peak moves inshore to $x \sim 80 \mathrm{~m}$, with a smoother distribution of velocity in the bar-trough region. Velocity strength over the bar-crest decreases from $0.7 \mathrm{~ms}^{-1}$ for $\alpha_{1}=0$, to $0.5 \mathrm{~ms}^{-1}$ for $\alpha_{1}=1$. The offshore velocity ( $\mathrm{x}>200 \mathrm{~m}$ ) values do not change significantly by changing the roller contribution as roller/breaking dissipation offshore of the bar-crest is negligible (Fig. 6a). 
Interestingly, using the mean normalized rms error from all seven stations, the results from Run 2 (VF model with no effect of rollers/wave-induced mixing) show the best overall agreement with the observations. Considering the variability observed in model performance at different cross-shore locations, it is clear that inclusion of wave rollers provides better agreement of longshore and cross-shore flows at the bar-crest and bar-trough region, but at locations further offshore, simulations with no rollers/mixing effects show a better agreement to observed profiles. These findings suggest that inclusion of processes like wave rollers requires careful definition of the amount of wave-dissipation responsible for driving the wave roller model (i.e., value of $\alpha_{r}$ ). Furthermore, it appears more sensible for this value to be a function of the cross-shore position within the surf zone (see Cambazoglu and Haas, 2011).
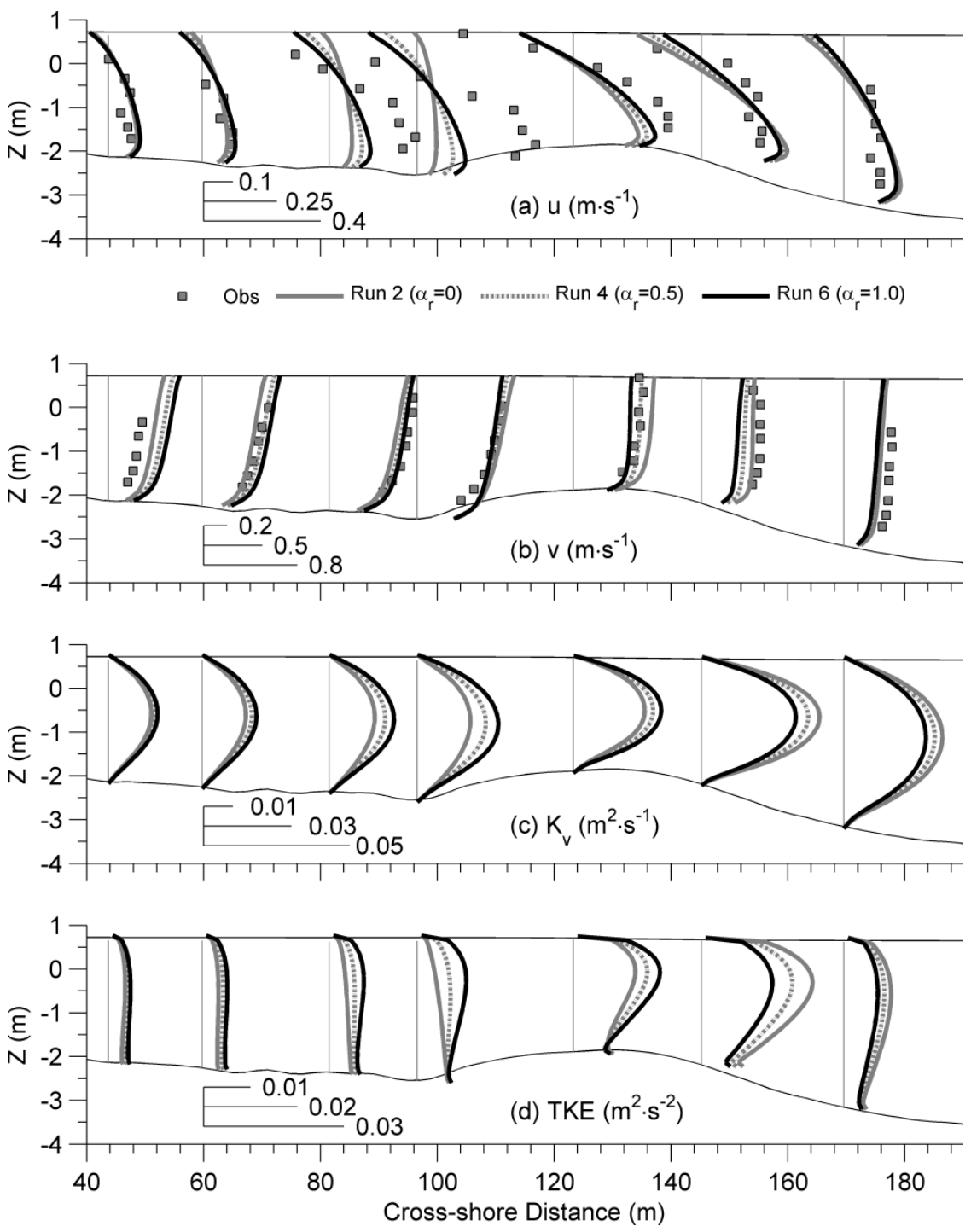

Figure 7. Comparison of model results (Runs 2, 4 and 6; i.e., VF model with $\alpha_{r=} 0,0.5$ and 1 , respectively) with observed vertical profiles (grey squares) of cross-shore (a) and longshore (b) velocities. Vertical grey lines indicate profile measurement locations and zero value for each profile (Data from Garcez-Faria et al. 1998; 2000). Vertical structure of eddy viscosity (c), $K_{v}$ and turbulent kinetic energy (d), TKE from model simulations at the same cross-shore locations as the velocities. 
The effect of wave rollers on the vertical distribution of vertical mixing $\left(K_{v}\right)$ and turbulent kinetic energy (TKE) is also examined using the same runs as those described in the previous paragraphs. At $\alpha_{r}=0$ (Run 2), the strongest velocity shear is observed offshore of the bar-crest (Fig. 7a), which corresponds to an increased region of TKE production and increased $K_{v}$ levels (Figs. $7 \mathrm{c}$ and d). As the roller contribution increases, the velocity shear at the bar-crest reduces, while an increase in velocity shear further inshore is observed. This is reflected by a decrease in TKE and $K_{v}$ (Figs. 7c and d) at the bar-crest and spreading of the TKE in the region between bar-crest and trough. Subsequently the vertical mixing within the bar-trough region also starts increasing. Overall, the roller contribution modifies the shear production and associated TKE and $K_{v}$, by moving the entire pattern further inshore and dispersing the breaking induced energy transformation more uniformly within the surf zone. It is interesting to point out that the $K_{v}$ values obtained in the present case are almost twice the magnitude of those used by U10 in their simulations. This occurs because the GLS mixing utilizes the ambient flow field to create the shear production and associated eddy viscosity profiles, while in U10 the $K_{v}$ values were derived using a K-profile parameterization. These differences in $K_{v}$ values also explain the small differences between results obtained by $\mathrm{U} 10$ and the present work.

\section{Effect of wave-induced mixing}

Wave-induced mixing is provided as a surface flux of TKE in the GLS turbulence closure scheme (see Eqns. 42-47, also Feddersen and Trowbridge, 2005), controlled by the empirical parameter $c_{\varepsilon w}$ that modifies the contribution from the breaking and roller dissipation. Feddersen and Trowbridge (2005) suggested a value of 0.25, while Jones and Monismith (2008) used a value of 0.06 for their simulations. We carried out a limited in scope sensitivity analysis by using $c_{\varepsilon w}=0,0.01$ and 0.05 for Runs 6, 9, and 10, respectively, all of which correspond to VF model based simulation with roller contribution of $\alpha_{1}=1.0$ (see Table 3). Simulated profiles of crossshore and longshore velocity from these runs are compared to field measurements (Figs. 8a and b), and the normalized rms errors are shown in Table 4. Simulations conducted using a $c_{\varepsilon w}=0.25$ (not shown here) significantly increase the $K_{v}$ and vertically mix the entire water column, destroying the vertical structure in cross-shore and longshore velocities.

The surface TKE flux increases the total TKE within the water column (Fig. 8d), developing a maximum value at the bar-crest and the shoreward boundary where the total dissipation is greatest. The vertical mixing $\left(K_{v}\right)$ shows a corresponding increase (Fig. 8c). When $c_{\varepsilon w}=0$ (i.e., Run 6), $K_{v}$ values of approximately $0.03 \mathrm{~m}^{2} . \mathrm{s}^{-1}$ are found over the bar-crest; and for $c_{\varepsilon w}$ values of 0.01 and 0.05 , these values subsequently increase to 0.05 and $0.06 \mathrm{~m}^{2} . \mathrm{s}^{-1}$ respectively (Fig. 8c). Similar increases in $K_{v}$ are also seen for locations further offshore of the bar-crest and over the bar-trough.

In general, increasing the surface TKE flux begins to destroy the vertical shear and the associated circulation pattern observed in the cross-shore and longshore velocities (Figs. 8a and b). In comparison to Run 6 (VF model with $\alpha_{l}=1.0$ and no wave mixing), the simulated crossshore velocity profiles from Run 9 and 10 (VF model with $\alpha_{r}=1.0, c_{\varepsilon w}=0.01$ and 0.05 , respectively) show higher rms errors at locations within the region between bar-trough and barcrest, and smaller errors at the station further offshore (see Table 4). The comparison of simulated and measured longshore velocity profiles (Fig. 8b) suggests that enhanced wave mixing (Runs 9 and 10) reduces the flow magnitude. This reduction deteriorates the agreement of model results to field observations at most of the measurement positions (see Table 4). Simulations conducted using $\alpha_{r}=0.5$ (not shown here) have shown similar response to that 
discussed here. We feel this is a typical response in cross-shore and longshore velocity field to increased mixing.

In Figures $7 \mathrm{a}, 7 \mathrm{~b}, 8 \mathrm{a}$ and $8 \mathrm{~b}$ the simulated and measured cross-shore and longshore velocity profiles are compared. Overall, the normalized rms errors obtained in these simulations vary between 0.54 and 0.66 for the cross-shore velocities and 0.20 to 0.3 for the longshore velocities. These values are similar to those of Newberger and Allen (2007b) and at times slightly higher than those shown by U10 (0.42-0.70 and 0.10-0.4 for cross-shore and longshore velocities, respectively). Nevertheless, our simulations show that the model is capable of creating realistic velocity profiles in a surf zone environment. In the remainder of the presentation, we focus on results from Run 6 (VF model with $\alpha_{r}=1$ and no wave mixing) as these simulated profiles show the best agreement to the observed cross-shore and longshore velocity measurements at the majority of the locations.
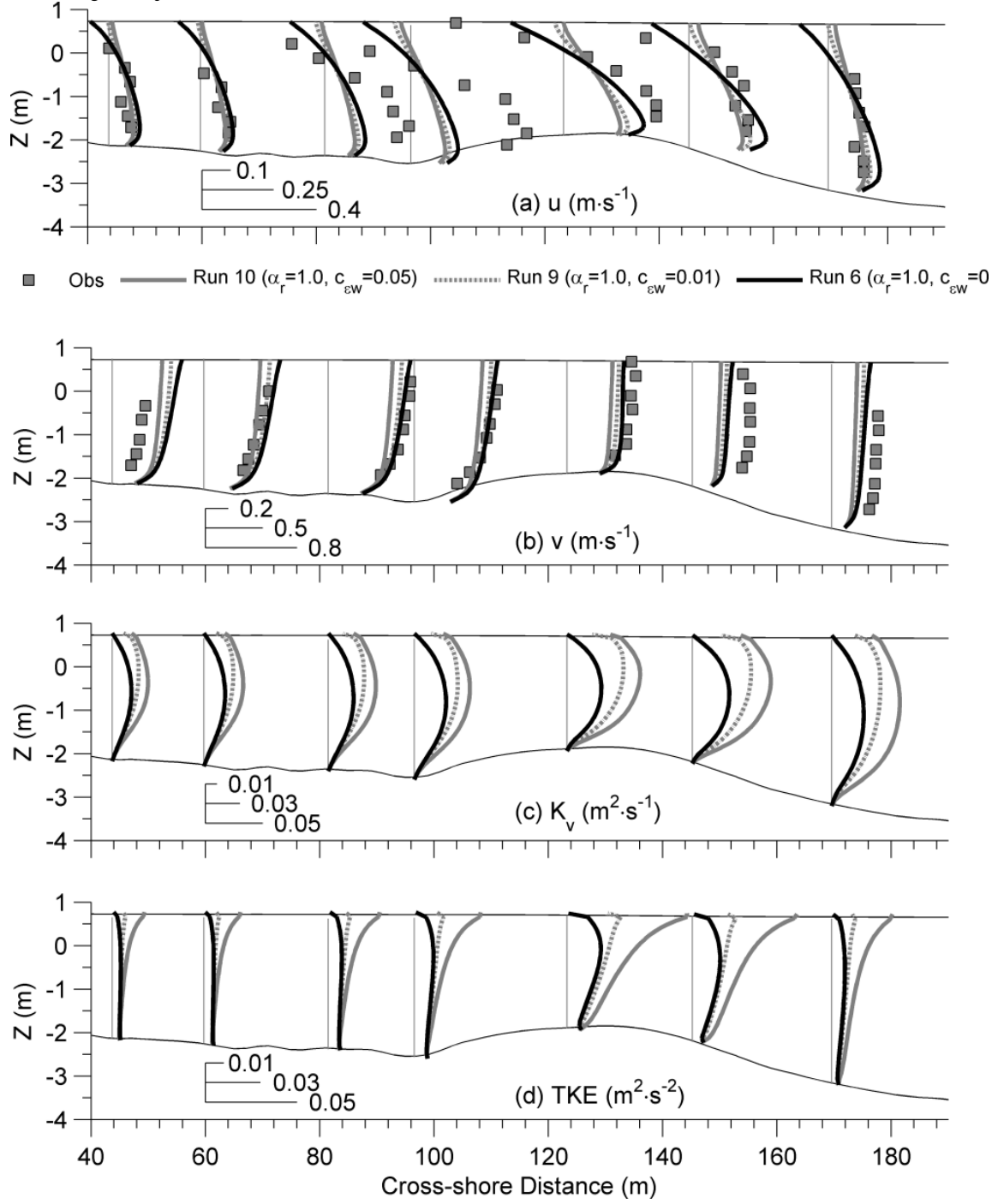

Figure 8. Comparison of model results (Runs 6, 9 and 10; VF model with rollers, $\alpha_{r}=1$ and wave-induced mixing with $c_{\varepsilon w}=0,0.01$ and 0.05 , respectively) with observed vertical profiles (grey squares) of cross-shore (a) and longshore (b) velocities. Vertical grey lines indicate profile measurement locations and zero value for each profile (Data from Garcez-Faria et al. 1998; 2000). Vertical structure of eddy viscosity (c), $K_{v}$ and turbulent kinetic energy (d), TKE model simulations at the same cross-shore locations as the velocities. 


\begin{tabular}{|c|c|c|c|c|c|c|c|c|c|c|}
\hline & \multicolumn{10}{|c|}{ Normalized Root Mean Square Error Analysis } \\
\hline & $\begin{array}{l}\text { RUN \# } \\
\text { STN \# }\end{array}$ & 2 & 3 & 4 & 5 & 6 & 7 & 8 & 9 & 10 \\
\hline \multirow{8}{*}{ 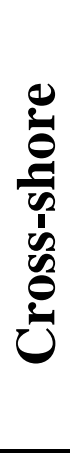 } & 1 & 0.80 & 0.80 & 0.80 & 0.80 & 0.79 & 0.88 & 0.92 & 0.87 & 0.92 \\
\hline & 2 & 0.82 & 0.80 & 0.77 & 0.75 & 0.73 & 0.83 & 0.88 & 0.80 & 0.86 \\
\hline & 3 & 0.74 & 0.68 & 0.62 & 0.58 & 0.53 & 0.69 & 0.72 & 0.63 & 0.67 \\
\hline & 4 & 0.84 & 0.76 & 0.71 & 0.66 & 0.62 & 0.76 & 0.78 & 0.70 & 0.73 \\
\hline & 5 & 0.46 & 0.43 & 0.41 & 0.39 & 0.37 & 0.65 & 0.71 & 0.62 & 0.69 \\
\hline & 6 & 0.44 & 0.42 & 0.40 & 0.38 & 0.37 & 0.42 & 0.46 & 0.44 & 0.48 \\
\hline & 7 & 0.47 & 0.45 & 0.43 & 0.41 & 0.39 & 0.25 & 0.17 & 0.22 & 0.15 \\
\hline & Mean & 0.65 & 0.62 & 0.59 & 0.57 & 0.54 & 0.64 & 0.66 & 0.61 & 0.64 \\
\hline \multirow{8}{*}{ 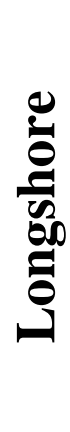 } & 1 & 0.52 & 0.66 & 0.79 & 0.89 & 0.98 & 0.65 & 0.43 & 0.82 & 0.59 \\
\hline & 2 & 0.10 & 0.05 & 0.08 & 0.13 & 0.17 & 0.07 & 0.17 & 0.11 & 0.10 \\
\hline & 3 & 0.13 & 0.10 & 0.07 & 0.06 & 0.05 & 0.13 & 0.23 & 0.10 & 0.19 \\
\hline & 4 & 0.14 & 0.12 & 0.11 & 0.10 & 0.11 & 0.10 & 0.15 & 0.12 & 0.16 \\
\hline & 5 & 0.22 & 0.14 & 0.08 & 0.08 & 0.13 & 0.08 & 0.17 & 0.20 & 0.30 \\
\hline & 6 & 0.11 & 0.18 & 0.25 & 0.31 & 0.37 & 0.33 & 0.43 & 0.43 & 0.53 \\
\hline & 7 & 0.22 & 0.25 & 0.27 & 0.30 & 0.32 & 0.33 & 0.45 & 0.37 & 0.49 \\
\hline & Mean & 0.21 & 0.21 & 0.23 & 0.27 & 0.31 & 0.24 & 0.29 & 0.31 & 0.34 \\
\hline
\end{tabular}
and longshore velocity estimates for DUCK' 94 for various locations across the profile and the different model simulations (Runs 2-10, see Table 3). $\mathrm{d}_{\mathrm{ij}}$ and $\mathrm{m}_{\mathrm{ijk}}$ represent measured (from Garcez-Faria et al., 1998, 2000) and model estimated velocity values at the 7 cross-shore locations (j) and various elevations (i) above the sea bed (for measurement locations see Fig. 10). Station 1 is closest to the shoreline. Numbers in bold typeface indicate minimum values.

Table 4. Normalized root mean square error $\varepsilon_{n r m s}(j, k)=\left\{\frac{\sum_{i=1}^{n}\left(d_{i j}-m_{i j k}\right)^{2}}{\sum_{i=1}^{n}\left(d_{i j}\right)^{2}}\right\}^{0.5}$ for the cross-shore

886

8

The horizontal and vertical distribution of the cross-shore velocity for Run 6 (VF model with $\alpha_{r}=1$ and no wave mixing) is shown in Figure 9a. As discussed previously, at the location of wave breaking, vertical segregation of flow occurs with an inshore directed flow at the surface and offshore directed flow at the bottom. Maximum strength of this undertow occurs at the barcrest and close to the shoreline, while relatively weaker values are found in the bar-trough. Outside the surf zone, flow through significant part of the water column is directed offshore with a maximum flow at the bottom layer, decreasing monotonically to a small onshore directed value at the sea surface (Fig. 9a). Maximum longshore velocity (Fig. 9b) occurs over the bar-trough with a smooth variation in the trough-crest region due to the effect of wave rollers. The strongest flow occurs at the surface, decreasing with an increase in the water depth. Further offshore of the bar-crest, longshore velocity decreases significantly, and most of the modeled longshore flow is 
wind driven. The vertical velocity (Fig. 9c) is directed downwards inshore of the bar-crest and upwards offshore of the bar-crest $(\mathrm{x}=130 \mathrm{~m})$. This pattern along with inshore flows at the surface and offshore directed flow in the center of the water column creates an anticlockwise circulation cell pattern which is similar to that found in the planar beach case presented in section 4.1 (see Fig. 2c).
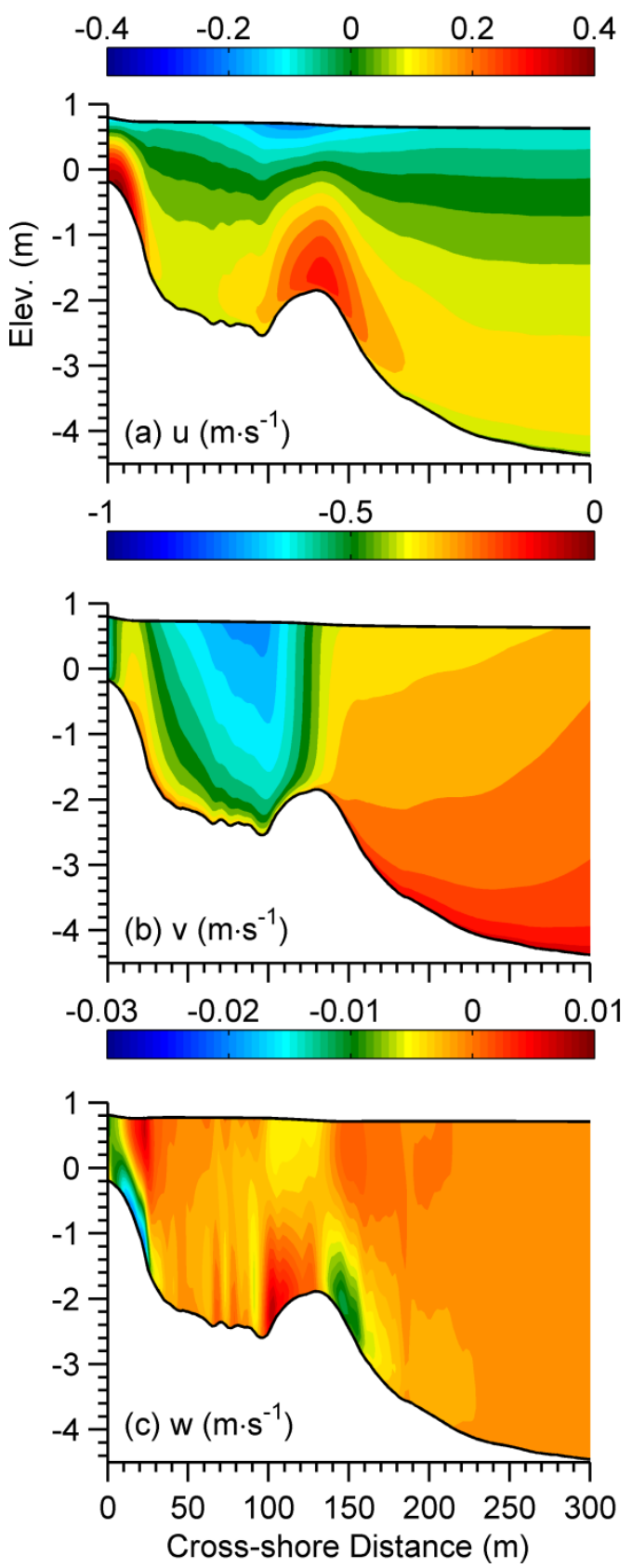

$-0.25-0.2-0.15-0.1-0.05$
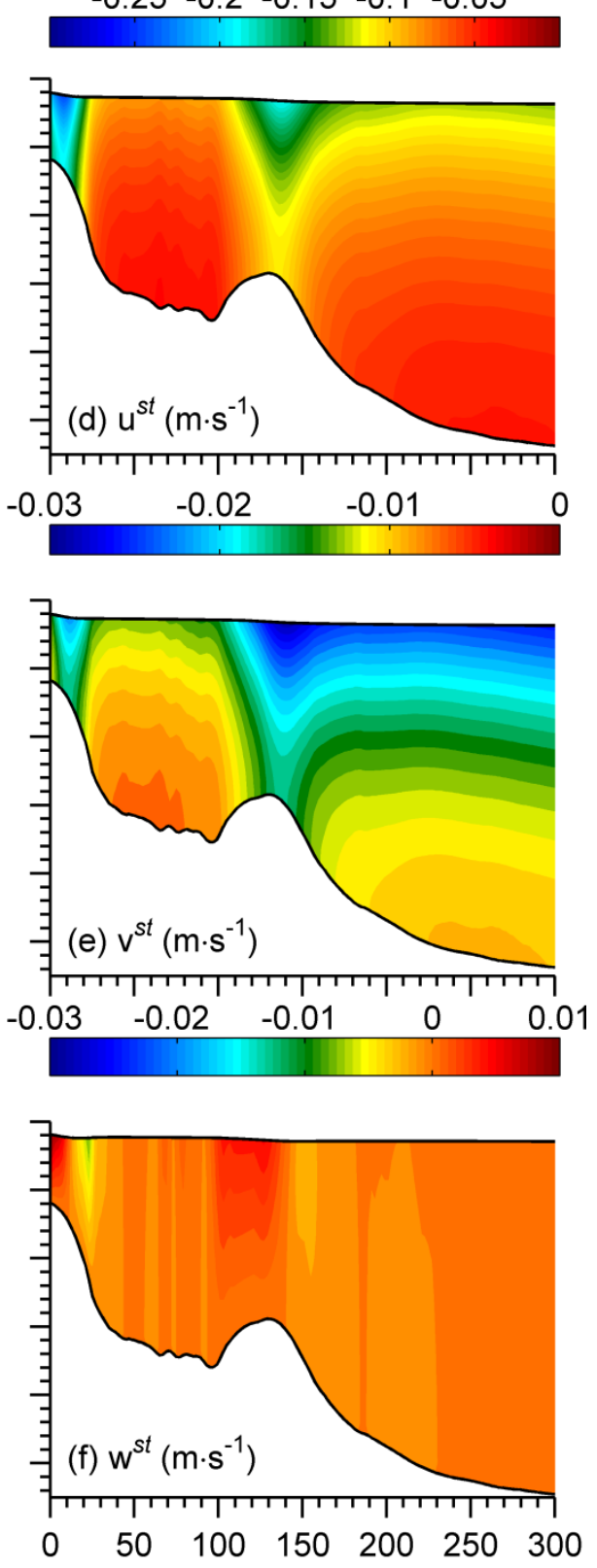

Figure 9. Cross-shore sections showing horizontal and vertical variability of Eulerian and Stokes velocity components for Run 6 (VF model with wave rollers, $\alpha_{r}=1$ and no wave mixing). (a) cross-shore $(u)$; (b) longshore $(v)$; and (c) vertical $(w)$ Eulerian velocities; (d) Cross-shore $\left(u^{s t}\right)$; (e) longshore $\left(v^{s t}\right)$; and (f) vertical $\left(w^{s t}\right)$ Stokes velocity. 
The vertical distribution of wave-induced Stokes drift follows a $\cosh (2 \mathrm{kz})$ distribution, with strongest flow near the surface and weakest flow near the sea bed. Maximum cross-shore and longshore velocities occur over the bar-crest and at very shallow waters further inshore (Figs. 9d and e). The cross-shore velocity is stronger than the longshore velocity, while the vertical Stokes velocity (Fig. 9f) is of similar strength as its Eulerian mean counterpart. As the flux divergence of longshore and cross-shore Stokes velocities is zero over the bar-crest, the vertical Stokes flow changes sign at this point. The upward and downward flow structure in the present case is opposite in sign to Eulerian mean flows (Fig. 9c). Presence of a vertical structure in water depth $<1 \mathrm{~m}$, also confirms presence of a vertically varying VF.

\subsubsection{Three-dimensional momentum balance}

The cross-shore and vertical variation of momentum balances for the VF simulation with wave roller action enabled ( $\left.\alpha_{r}=1\right)$ and no wave mixing (Run 6) is shown in Figure 10. In the cross-shore direction the horizontal momentum balance (see Eqns. 11 and 12) is dominated by the roller acceleration (BA, Fig. 10a), pressure gradient (PG, Fig. 10d) and vertical mixing (VM, Fig. 10e). The horizontal advection (HA, Fig. 10b), horizontal vortex force (HVF, Fig. 10d) and vertical advection (VA, Fig. 10f) terms are insignificant. The BA is surface intensified (Fig. 10a) with strongest values occurring at locations where total wave dissipation is maximum. At the surface layer, the BA is balanced by the sum of VM and PG (Figs. 10a, c and e), while further below (D > 1 m), BA becomes negligible and PG is balanced by VM (Fig. 10e). Similar balance is also observed at the shoreward boundary. This cross-shore momentum balance is similar to that observed for the planar beach example in section 4.1.3.

Analysis of the longshore momentum balance shows that with the exception of PG all remaining terms (i.e., BA, VM, HA, VA and HVF) are significant. The sum of BA and HA terms (Figs. $10 \mathrm{~g}$ and $\mathrm{h}$ ) is balanced by the sum of VM, VA and HVF (Figs. 10k, $\mathrm{h}$ and l, respectively). BA (Fig. 10g) is strongest in the surface layer over the bar-crest/trough region and near the shoreline and balanced primarily by the HVF term (Fig. 10i). It is noticeable that at these locations of strong BA contribution, VM takes its smallest values. However near the surface and in the region between the bar-crest and shoreline, the VM term becomes more significant. In addition, near the bed the VM term is largest over the bar-crest and together with HVF (Fig. 10i) balance HA (Fig. 10h). It is noticeable that over the bar-crest BA is balanced mainly by HVF, in the absence of a bar (see planar beach case) BA is balanced by VM.

At this stage it is important to point out that a traditional alongshore momentum balance in a radiation stress approach suggests that gradient of radiation stress $\left(\partial S_{x y} / \partial x\right)$ is balanced by VM (see HW09). In the present case, a summation of HA (Fig. 11h), HVF (Fig. 11i) and VA (Fig. 11i) is small and dominant balance is between BA and VM at most of the cross-shore locations, i.e., similar to radiation stress approach. However, HA and HVF do not completely cancel each other and have a net-contribution in modifying the flow pattern (see Sec. 5).

\subsubsection{Balance of vertically-integrated three-dimensional momentum balance}

The two-dimensional momentum balance in the cross-shore direction (Fig. 11a) demonstrates a balance between pressure gradient (PG) and the breaking /roller acceleration (BA) terms. In the longshore direction the major contributors are vortex forces (VF), horizontal advection (HA), breaking accelerations (BA) and bottom stress (BStr), as was the case for a 
planar beach (Fig. 4b). It is noticeable that due to non-planar variation in bathymetry in this case, the relative contribution of each term is different than that found for the planar beach case, and the HA and HVF (Fig. 11b) are not symmetrical anymore.
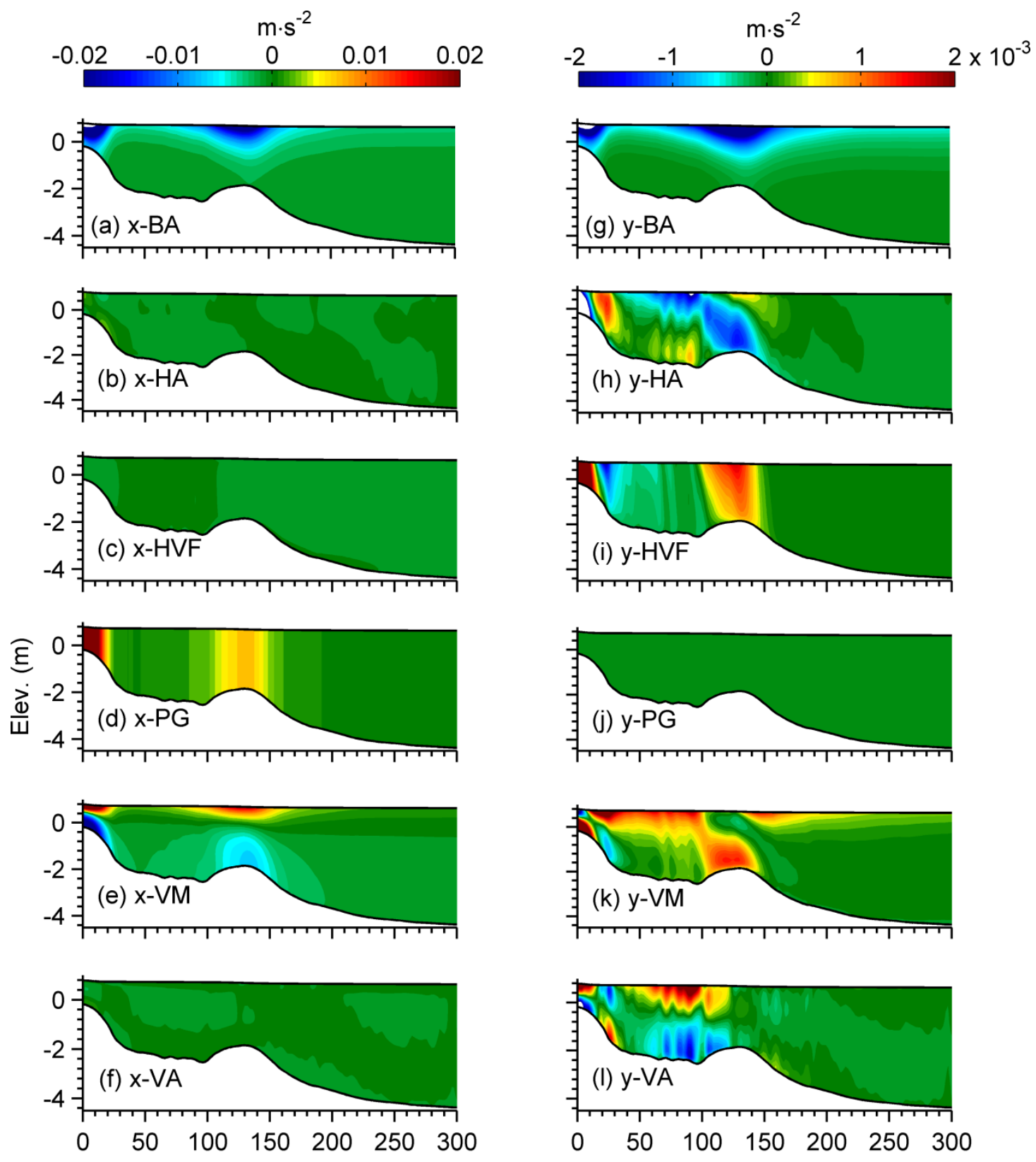

Cross-shore Distance (m)

Figure 10. Cross-shore and vertical distribution of the terms contributing to the cross-shore (x) and longshore (y) momentum balance for Run 6 (VF model with wave rollers, $\alpha_{r}=1$ and no wave mixing). Cross-shore terms: (a) x-breaking acceleration (x-BA) ; (b) Eulerian, x-horizontal advection (x-HA); (c) xhorizontal vortex force (x-HVF); (d) x-pressure gradient (x-PG); (e) x-vertical mixing (VM); (f) x-vertical advection (VA); and alongshore terms: (g) y-breaking acceleration (y-BA); (h) Eulerian, y-horizontal advection (y-HA); (i) y-horizontal vortex force (y-HVF); (j) y-pressure gradient (y-PG); (k) y-vertical mixing (y-VM); and (l) y-vertical advection (y-VA). 
Decomposing the pressure gradient force into individual components (Eqn. 49) shows that the Eulerian response, $P_{c x}$ is the major contributor (Fig. 11c). Unlike the planar beach, the Bernoulli head $\left(P_{b h x}\right)$ plays an important role over the bar-crest and further inshore. This occurs because Bernoulli head is dependent upon the velocity shear, and in this example high velocity shear is present in the region between the trough and crest of the bar. The quasi-static response $\left(P_{q s x}\right)$ also becomes dominant at the bar-crest and adds to $P_{b h}$, while the surface pressure boundary correction $\left(P_{p c x}\right)$ is negligible.
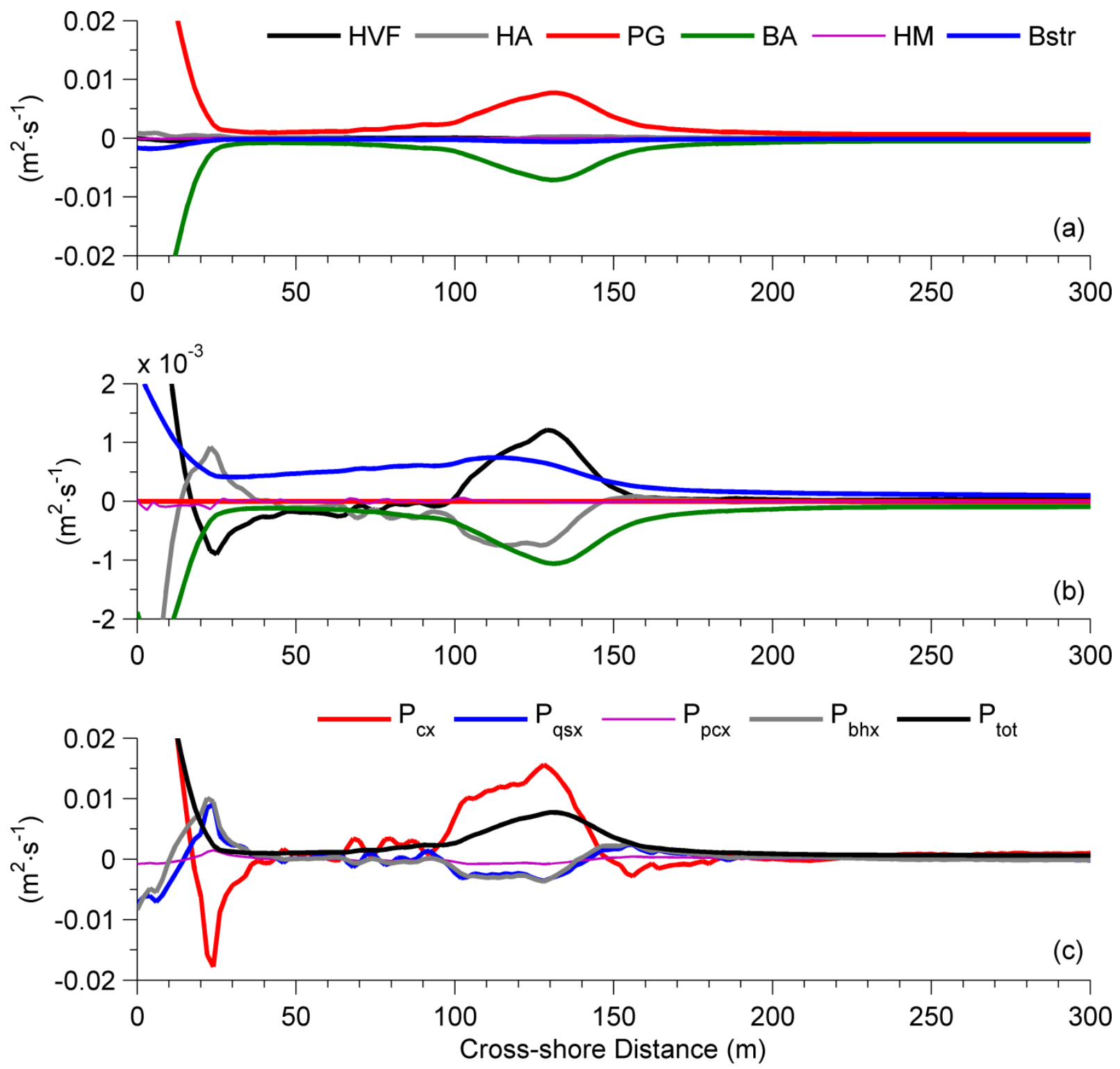

Figure 11. Cross-shore variation of depth-averaged (a) cross-shore and (b) longshore momentum balance terms. (c) Decomposed PGF terms in cross-shore as described in Eqn. 49 for Run 6 (VF model with wave rollers, $\alpha_{r}=1$ and no wave mixing). 


\subsection{Test Case 3: Nearshore barred morphology with rip channels}

This case investigates the dynamics of a barred beach bathymetry that develops rip currents for normally incident waves. The application is based on a laboratory scale experiment and is similar to a case demonstrated in HW09, with a few major differences: (a) in HW09 the wave driver was a monochromatic wave model (REF/DIF), while here we use a spectral wave model (SWAN); (b) the HW09 domain was identical to the laboratory experiments while our domain has been scaled by a factor of 20 (kinematic similarity, Hughes, 1993) to create more realistic field conditions (similar scale as Aagaard et al., 1997; Macmahan et al., 2005; ); and (c) bottom friction due to combined action of waves and currents (Madsen, 1994, also see section 4.2.1) is used instead of a logarithmic bottom drag.

The bathymetry domain (Fig. 12a) is an idealized version of that used by Haller et al. (2002) and Haas and Svendsen (2002). The scaling of the domain by a length scale, NL=20, leads to a maximum depth of $10 \mathrm{~m}$, a nearshore bar of $1.20 \mathrm{~m}$ located $80 \mathrm{~m}$ off the coastline, cross-shore domain width of $292 \mathrm{~m}$ and alongshore length of $524 \mathrm{~m}$. To avoid interaction of rip channel flow with the lateral boundaries, the domain was extended laterally by $80 \mathrm{~m}$ in either direction. Rip channels are spaced $184 \mathrm{~m}$ apart and the channel width is $36.4 \mathrm{~m}$ which makes the ratio of channel width to rip current spacing 0.2 , a value consistent with those found in the field (e.g., Huntley and Short, 1992). The model grid has a horizontal resolution of $2 \mathrm{~m}$ in both directions and consists of 20 equally spaced sigma layers. The boundary conditions at shoreline, offshore boundary and lateral ends are no flow conditions (i.e., closed boundary conditions at the coast, lateral boundaries and offshore) and are the same as the laboratory experiments of Haller et al., (2002). Since the effect of wave rollers is important in a surf zone environment (see section 4.2.2), we use a $\alpha_{r}=0.5$ to allow for $50 \%$ contribution of roller acceleration to momentum balance. In order to maintain realistic conditions, enhanced mixing due to wave breaking is also considered with a $c_{\varepsilon w}=0.02$.

At the offshore boundary, SWAN is forced with $1.0 \mathrm{~m}$ waves $\left(H_{\text {sig }}\right)$ with peak period of $6.3 \mathrm{~s}$, and directional spreading of $8^{\circ}$ propagating perpendicular to the shoreline. From these values, SWAN computes a wave spectrum based on a JONSWAP distribution. The spectral resolution is 20 frequency bands in the frequency range between $0.04 \mathrm{~Hz}$ and $1 \mathrm{~Hz}$, and 36 directional bins of $10^{\circ}$ each from $0^{\circ}$ to $360^{\circ}$. A depth induced breaking constant of $\gamma_{b}=0.6$ is chosen to account for depth limited wave breaking (Battjes and Janssen, 1978; Eldeberky and Battjes, 1996), while the eddy viscosity model of Madsen (1988) for bottom friction induced wave attenuation is used with a bottom friction roughness length scale of $0.05 \mathrm{~m}$. Because of the high spatial resolution of the domain a time step of $0.5 \mathrm{~s}$ is used for both ROMS and SWAN ${ }^{2}$ while the coupling between the two models takes place every $5 \mathrm{~s}$. Comparisons are shown after 1 hour of simulation time. In order to make our results comparable to those presented in HW09 and Kumar et al. (2011a) a relatively higher horizontal mixing coefficient $\left(0.20 \mathrm{~m}^{2} \mathrm{~s}^{-1}\right)$ has been used that leads to relatively stable flows. The ability of the model to simulate the unstable character of rip currents (e.g., Haas and Svendsen, 2002) is demonstrated through the

\footnotetext{
${ }^{2}$ A time step of $0.5 \mathrm{~s}$ leads to a CFL number of $\sim 1$. Trial runs with larger time steps in SWAN (1, 2, 3 and $\left.5 \mathrm{~s}\right)$ when compared with the $0.5 \mathrm{~s}$ time step run revealed overall RMS differences in wave height of $0.34,0.83,0.81$ and $0.66 \%$ respectively, while the RMS difference in vorticity was $0.009,0.013,0.021$ and $0.044 \%$ respectively. These differences become larger for smaller water depths $(1.12,2.30,2.23$ and $1.96 \%$ for wave height and $0.031,0.045$, 0.072 , and 0.16 for vorticity for water depths less than $0.5 \mathrm{~m}$ ) This suggests that although the internal limiter in SWAN (Ris, 1999) is effective in making the wave model stable, it does not completely eliminate inaccuracies in the wave results due to large time steps, but the overall differences are found to be relatively small.
} 
presentation of a case where a lower, more realistic horizontal mixing coefficient is used $(0.05$ $\mathrm{m}^{2} \mathrm{~s}^{-1}$ ).

\subsubsection{Wave parameters and sea surface elevation}

At the rip channel locations, wave - rip current interaction (Fig. 12b) causes a local increase of wave steepness. Greater water depths at the channel locations allow for further inshore propagation of these incoming waves, which finally start breaking at $\mathrm{x} \sim 50 \mathrm{~m}$. On the other hand, waves propagating over the bar start breaking at $\mathrm{x} \sim 70 \mathrm{~m}$, become stable $(25 \mathrm{~m}<\mathrm{x}<$ $65 \mathrm{~m})$ and then break again near the shoreward boundary $(\mathrm{x}<25 \mathrm{~m})$. The difference in wave breaking pattern over the channel and the bar creates a lateral difference in breaking induced wave set-up at these two locations.
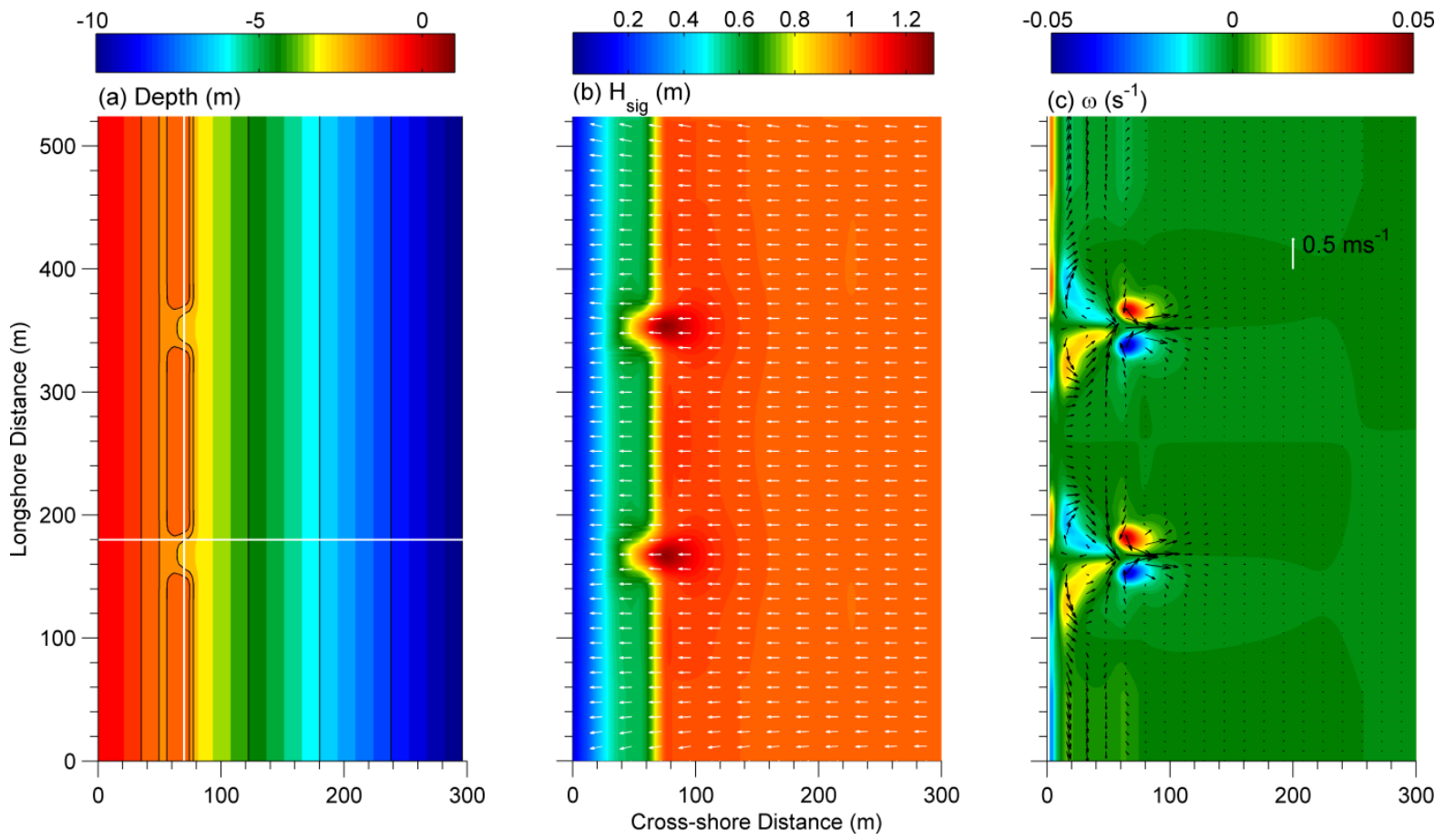

Figure 12. Rip channel case. (a) Bathymetric domain; (b) significant wave height (contours) and direction (arrows); and (c) vorticity vector after 1 hour of model simulation. Black arrows in (c) show the depth averaged, Eulerian velocity vector. The white line in (c) shows velocity strength of $0.5 \mathrm{~ms}^{-1}$. The solid white lines in (a) show the transects along which cross-shore and longshore momentum balances are described in Figures 15 to 16.

\subsubsection{Nearshore Flows}

Differences in sea surface elevation due to wave set-up drive mean flow patterns. Higher wave-setup at the bar than the channel creates "feeder" currents directed towards the latter which results in a confluence of flow from both sides leading to the development of the outgoing rip current (Fig. 12c). Close to the shoreline, the wave set-up pattern reverses, as the larger waves within the rip channel break further inshore; this creates a higher wave set-up inshore of the channel in comparison to locations inshore of the bar. The waves in the latter location have 
already dissipated due to wave breaking over the bar. This wave-setup gradient causes alongshore flows inshore the bar, directed away from the channel (see Fig. 12c). Overall a primary circulation pattern develops with outgoing feeder currents from the rip channel and return flow over the bar, and a secondary circulation pattern close to the shoreline, with inshore flows directed towards the shoreline and longshore velocity directed away from the rip channel (Fig. 12c). Further offshore, the strength of the rip current gradually decreases until it becomes negligible. These simulated results are consistent with the laboratory studies conducted by Haller et al. (2002), Haas and Svendsen (2002) and the modeling work of Haas et al. (2003), Yu and Slinn (2003) and Kumar et al. (2011a). Flow vorticity vector contours (Fig. 12c) show two vortex patterns inshore and offshore of the rip channel, corresponding to the secondary and primary circulation patterns, respectively. Each vortex pattern consists of a pair of vortices of opposite signs, suggesting opposite circulation tendencies.
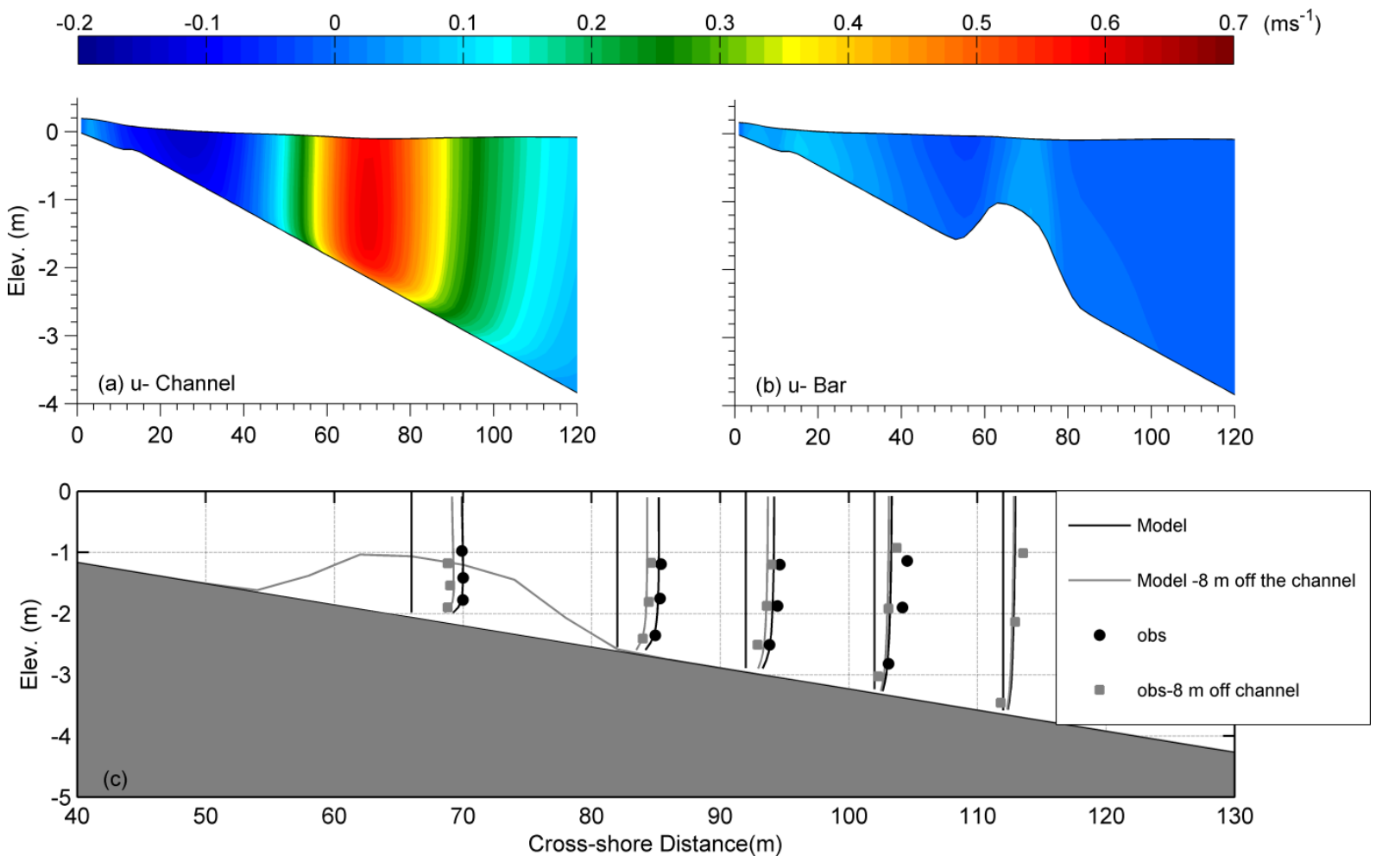

Figure 13. Vertical structure of cross-shore Eulerian velocity at (a) the center of rip channel and (b) over the bar. Results derived from $\mathrm{VF}_{3 \mathrm{D}}$ based model simulations. (c) Comparison of normalized model derived cross-shore velocity with normalized data from Haas and Svendsen, 2002 (key: symbols $\boldsymbol{\square}$ and $\boldsymbol{\square}$ and grey and black lines denote data and model results at the center and $8 \mathrm{~m}$ off the channel, respectively).

Vertical profiles of Eulerian mean cross-shore velocities in the rip channel and over the bar are shown in Figures 13a and b, respectively. At locations inshore of the rip channel $(x<40$ $m$, Fig. 13a) the flow is directed inshore from surface layer to the middle of the water column, while weak offshore directed flow is seen at the bottom layer. Inshore flow is strongest at the surface $\left(\sim 0.3 \mathrm{~ms}^{-1}\right)$ and decreases with depth. Within the rip channel and further offshore (40 m $<x<100 \mathrm{~m}$ ) the flow is directed seaward. Strongest offshore directed flow (of the order of 0.7 $\mathrm{ms}^{-1}$ ) occurs over the rip channel at $\mathrm{x} \sim 70 \mathrm{~m}$ and close to the middle of the water column with a 
monotonic decrease in magnitude with increasing or decreasing water depth. Inclusion of horizontal viscous mixing and wave-induced enhancement in mixing reduces the horizontal and vertical shear in velocity by dispersal of momentum, providing smoother solutions. In comparison to flows observed within the rip channel, the flow field is relatively weaker over the bar (Fig. 13b). Wave breaking occurs over the bar-crest and at the shoreward boundary. Undertow in the bottom layer with a magnitude of $\sim 0.3 \mathrm{~ms}^{-1}$ is observed at both breaking locations, while in the surface layer flow is directed towards the shore (Fig. 13b). Overall, the velocity profile observed over the bar is similar to that discussed earlier for the DUCK' 94 simulations.

Our scaled numerical experiment conditions correspond to Test B of Haller et al. (2002) and Test R of Haas and Svendsen (2002). We use the results of those lab experiments to provide a semi-quantitative comparison between the measured and modeled vertical structure of the cross-shore velocity field. For this comparison we use all of the bin averaged velocities from Test R (see Fig. 11 in Haas and Svendsen, 2002) and for all reported locations (Fig. 13c). The measured and simulated velocities are normalized by the corresponding maximum cross-shore velocity at the center of the rip channel, respectively. The simulated normalized cross-shore current vertical structure from the model simulation agrees well with the experimental data (Fig. 13c and Table 5). Inside the channel, rip current speed is greatest just below the level of the barcrest and decreases toward the surface and bed. However no experimental data are available near the surface. Just offshore the bar, the normalized data from the model simulation show a parabolic profile with stronger velocities at the center of the water column, while the experimental data suggest vertically decreasing magnitude of velocity in the water column. The rms error (normalized by maximum observed value, as in Sheng and Liu, 2011) is small within the rip channel (5.8\%, Table 5) but increases for locations further offshore (Table 5). The overall rms error is $12.7 \%$.

Table 5. The RMS error (normalized by the maximum observed value) for the simulated cross-shore velocities for nearshore barred beach with rip channels $(\sec 4.3)$.

\begin{tabular}{|c|c|}
\hline \multirow{2}{*}{ STN \# } & RMS Error (\%) \\
\cline { 2 - 2 } & Cross-shore Vel. \\
\hline 1 & 05.8 \\
\hline 2 & 05.3 \\
\hline 3 & 13.1 \\
\hline 4 & 28.3 \\
\hline 5 & 36.0 \\
\hline Overall & $\mathbf{1 2 . 7}$ \\
\hline
\end{tabular}

\subsubsection{Unstable rip current flow}

Rip currents are unstable in nature (Haas and Svendsen, 2002), and processes like vortex propagation and vortex shedding have been observed both in numerical simulations and field experiments (see Yu and Slinn, 2003; Haller and Dalrymple, 2001; MacMahan et al., 2005; Reniers et al., 2009). The importance of these vortices lays in the fact that they interact with the 
incoming wave-induced Stokes drift and create a strong VF in the longshore direction (negligible in cross-shore direction as $v^{S t}$ is almost zero), which may play a relevant part in the maintenance and advection of these vortices.
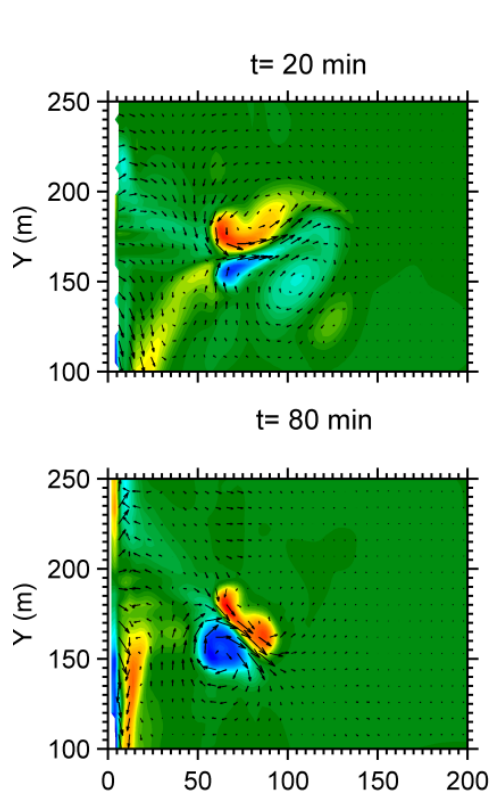

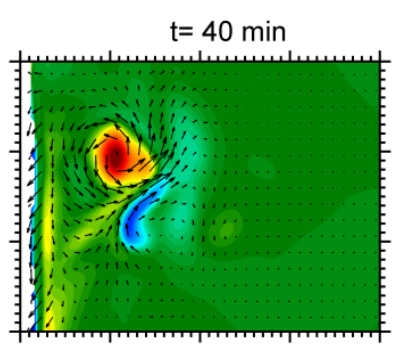

$\mathrm{t}=100 \mathrm{~min}$

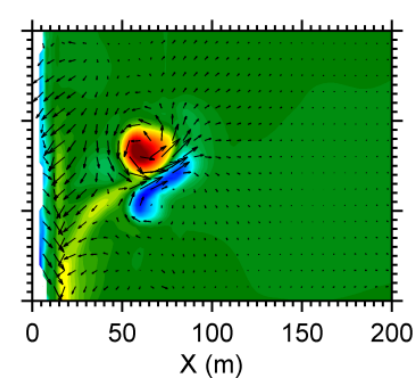

$\omega\left(s^{-1}\right)$
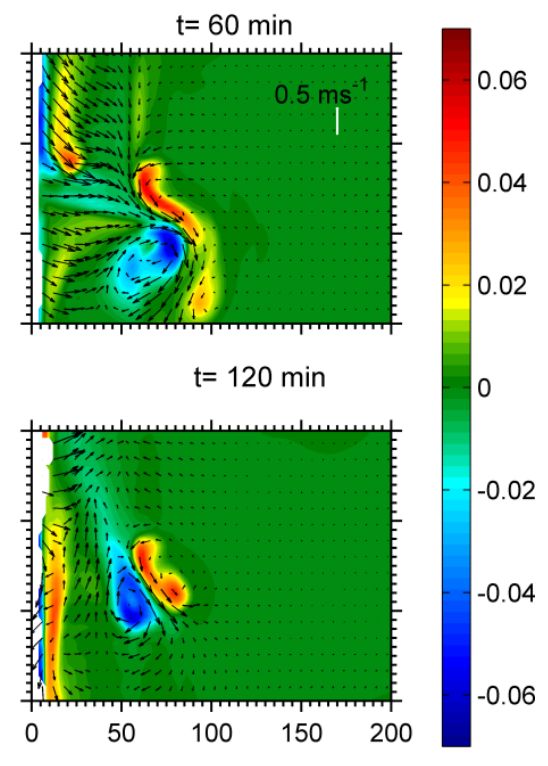

Figure 14. Example of unstable rip current conditions simulated using with a linear bottom friction $(\mu=0.002 \mathrm{~m})$ and a horizontal mixing of $0.05 \mathrm{~m}^{2} \mathrm{~s}^{-1}$. Snapshots of vorticity and depth-averaged, Eulerian velocity vector for six different time steps with a time interval of $20 \mathrm{~min}$. Only the computational domain in the vicinity of the rip channel is shown here.

The dynamics of a barred beach with rip channels for normally incident waves are investigated for the same model domain as in Fig. 14, and same offshore wave conditions. Unlike the previous simulation ( $\operatorname{Sec} 4.3$ ), in this case we use a linear bottom drag formulation with a drag coefficient of $0.002 \mathrm{~ms}^{-1}$ (Yu and Slinn, 2003) and a horizontal mixing coefficient of $0.05 \mathrm{~m}^{2} \mathrm{~s}^{-1}$. Snapshots of vorticity vector and mean flow (Fig. 14) show the evolution of flow vorticity over the computational domain. The direction of rip current is at an angle to the rip channel, and its strength changes over time. It is also interesting to see that the vorticity pattern has a periodicity of approximately 60 minutes, which agrees with previous model simulations of rip currents (Yu and Slinn, 2003).

\subsubsection{Three-dimensional momentum balance}

The three-dimensional momentum balance is presented along a cross-shore and a longshore transect. The cross-shore transect is defined by a line that passes through the center of a vortex (i.e., $y=180 \mathrm{~m}$, Fig. 12), as this is the region where the VF contribution is most significant. This transect is midway on the slope between the bar and the rip channel. The alongshore transect is at location $\mathrm{x}=70 \mathrm{~m}$ and it passes through the center of the rip channel (see Fig. 12).

The horizontal cross-shore momentum balance has a pattern which is similar to that presented for the planar and barred beach cases. PG, BA, HA and VM are the dominant terms, 
while HM and HVF are negligible (Figs. 15a to f). The BA term becomes important at $\mathrm{x}<90 \mathrm{~m}$, a location where wave breaking has just initiated within the rip channel while the majority of the waves break further inshore (Fig. 15a). As in the other cases, the influence of BA is limited to the sea surface and is balanced by VM. Since the domain is not alongshore uniform, advection becomes important. This is shown in Fig. 15b, where the HA contribution is significant on the shoreward side of the bar and when is added to VM, the sum balances the PG term.

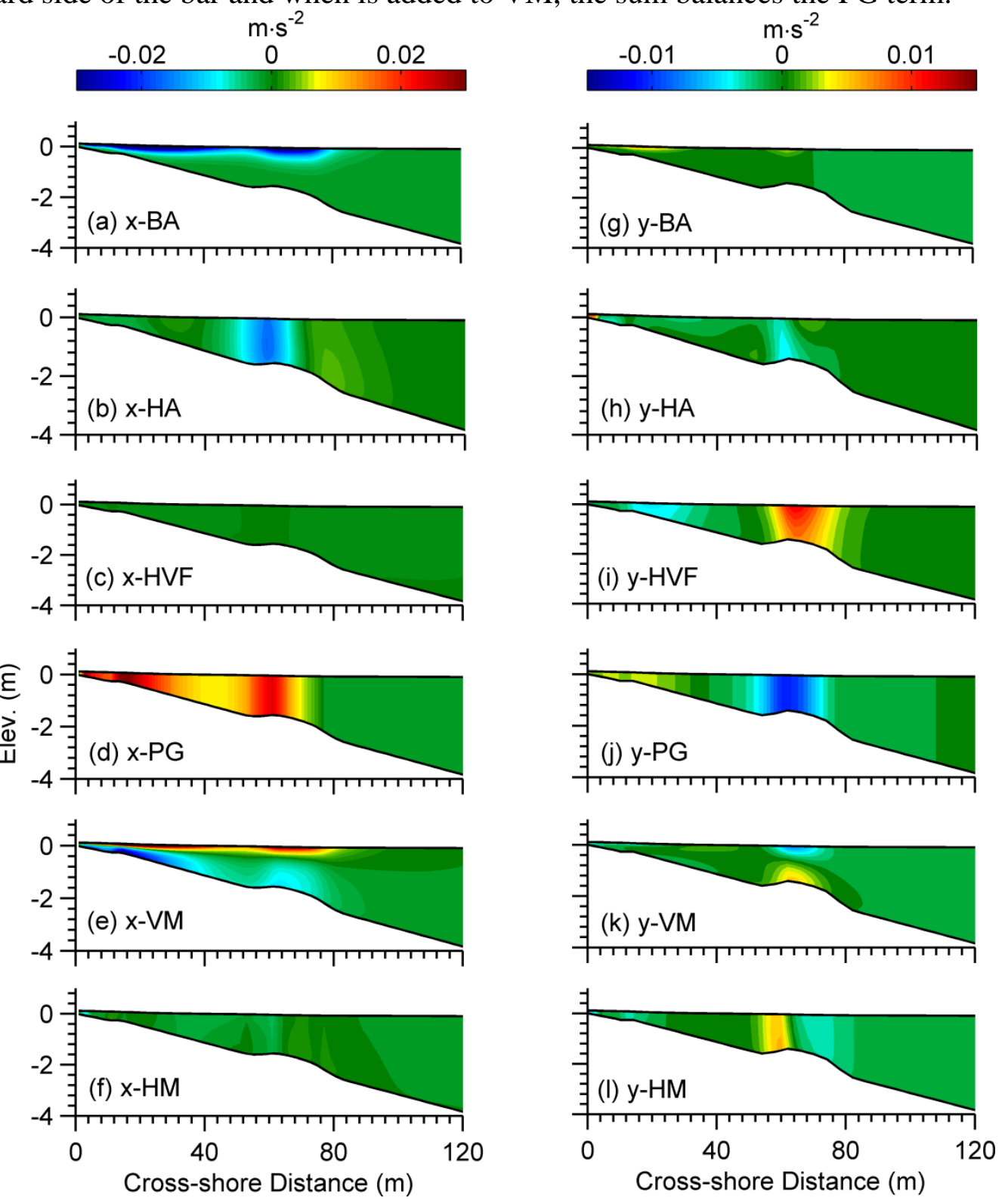


The longshore momentum balance analysis shows that PG, HA, HM, HVF and VM are the important terms while BA (Fig. 15g) is negligible. The feeder current developed near the rip channel (Fig. 15c) is driven by pressure gradients (PG, Fig. 15j) due to differences in wave setup levels over the bar and the channel location, respectively. This PG term is stronger in the vicinity of the bar and it is balanced predominantly by the HVF term (Fig. 15i) which is stronger near the sea surface and decreases toward the sea bed. It is near the bed where the positive values of VM (Figs. 15k) add to HVF to balance PG. Near the surface the negative values of VM add to PG to balance HVF. Finally, HA (Fig. 15h) is half the strength of PG and has similar magnitude and opposite sign as of HM (Fig. 15l).
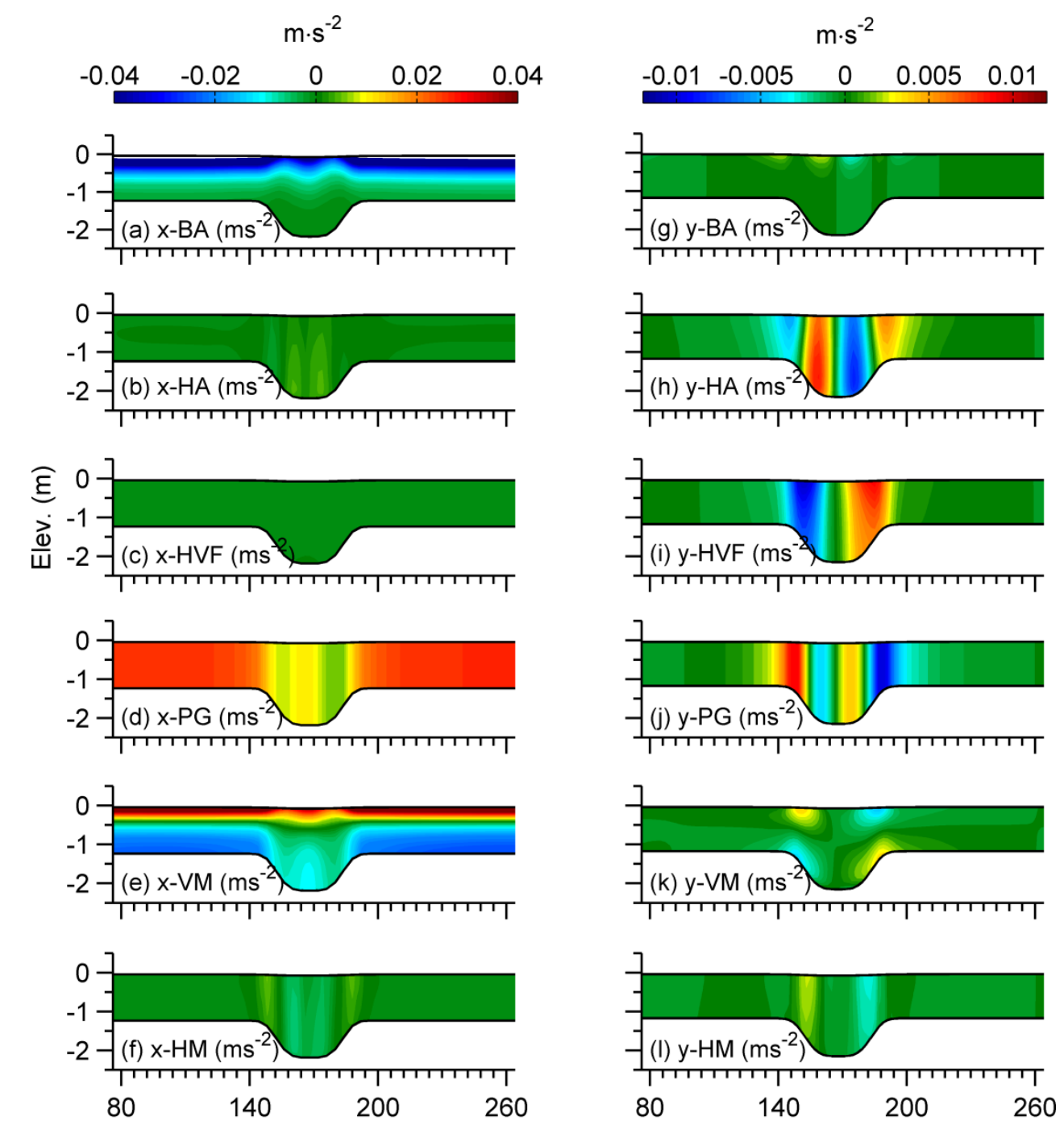

Longshore Distance $(\mathrm{m})$

Figure 16. Longshore distribution of vertical profile of contributing terms in cross-shore (x) and longshore (y) momentum balance at $\mathrm{x}=70 \mathrm{~m}$ (see Fig. 12 for transect location). Cross-shore terms: (a) $\mathrm{x}$-breaking acceleration ( $\mathrm{x}$ BA); (b) Eulerian, $\mathrm{x}$-horizontal advection (x-HA); (c) x-horizontal vortex force (x-HVF); (d) x-pressure gradient (xPG); (e) x-vertical mixing (x-VM); (f) x-vertical mixing (x-VM); Longshore terms: (g) y-breaking acceleration (yBA); (h) Eulerian, y-horizontal advection (y-HA); (i) y-horizontal vortex force (y-HVF); (j) y-pressure gradient (yPG); (k) y-vertical mixing (y-VM); and (l) y-horizontal mixing (y-HM); 
The longshore variation of the cross-shore momentum terms is shown in Figures 16a to f, where it is shown that the terms HA, HM and HVF (Figs. 16b, f and c, respectively) are insignificant. The intensity of depth-induced wave breaking over the bar-crest is higher than in the rip channel, except at the center of the channel due to wave focusing by the rip currents. This wave breaking pattern is reflected in the contribution of BA which is stronger over the bar, reduces at the channel sides and becomes strong again at the center of the channel (Fig. 16a). PG (Fig. 16d) is vertically uniform, and in response to BA it takes high values over the bar; at the channel center it takes values approximately $50 \%$ lower while at the channel sides it is further reduced to almost $25 \%$ of its value over the bar. Near the sea surface, the sum of VM (Fig. 16e) and PG balances BA, while in deeper waters within the channel, PG is balanced predominately by VM.

The longshore variability of the longshore momentum terms is primarily due to HA, PG, $\mathrm{HVF}$, and secondary due to VM and HM. The role of BA (Fig. 16g) is relatively insignificant and limited to the surface layer. HA (Fig. 16h) is zero at the center of the rip channel as no significant longshore velocity is present at this location. In the region 150 to $170 \mathrm{~m}$, the HA term is positive, while from 170 to $190 \mathrm{~m}$ it becomes negative. Two more inflexion points with zero HA are observed at longshore locations corresponding to the centers of the two vortices which are found just outside the rip channel (see Fig. 12c). As the sense of rotation of vorticity is opposite about the rip channel center, the sign of HA term changes accordingly (i.e., negative for $130 \mathrm{~m}<y<150 \mathrm{~m}$ and positive for $190 \mathrm{~m}<y<210 \mathrm{~m}$ ). The PG (Fig. 16j) term is vertically uniform and has the opposite sign of HA. It is important to note that PG has the same inflexion points as HA which are created by local changes in alongshore pressure gradient signs. These changes are attributed to different wave set-up levels generated by lateral variation of the wave height and associated breaking processes. Waves break over the bar on either side of the channel but also in the center of the channel at the location of maximum rip current. The latter occurs because wave-current interaction at the center of the channel is responsible for an increased wave height which initiates wave breaking at these larger depths. The HVF term (Fig. 16i) takes its maximum value at the locations with the strongest vorticity (Fig. 12c) and decreases with increasing water depth. It adds to HM (Fig. 161) and PG to balance HA inside the rip channel, while outside the channel it adds to HA to balance PG. HVF decreases toward the channel and it becomes zero at the channel center. Overall, the HA and HVF together preserve the flow vorticity created due to PG.

\subsection{Test Case 4: Wave-induced cross-shore flows in the inner shelf}

One of the justifications for implementing the VF formalism in COAWST was to develop a modeling system capable of a seamless transition from inner shelf and through the surf zone. Cases presented earlier have focused on surf zone processes and the case presented here aims at the region of shoaling waves outside the surf zone. In a recent study conducted by Lentz et al. (2008), observational data of undertow from Martha's Vineyard Coastal Observatory (MVCO) were used to show a strong correlation between depth-averaged Stokes drift and undertow outside the surf zone in water depths varying from 5-17 m. Furthermore, in calm wind conditions $\left(\tau_{s}<0.03 \mathrm{Nm}^{-2}\right)$ the profile of inner shelf cross-shore Eulerian mean flow was found to not be parabolic (as it has been found to be inside the surf zone, see Figs 8 and 9); instead a maximum offshore flow was observed at the surface, decreasing towards the bottom. In order to explain the observed velocity profiles, Lentz et al. (2008) presented a basic undertow model consisting of the 
following forces: (a) Hasselmann wave stress (Stokes-Coriolis force); (b) bottom streaming; (c) surface streaming; (d) Coriolis force; and (e) pressure gradient and wave shoaling.

In simulations conducted for similar conditions, we considered non-breaking waves so that $\mathcal{F}^{w}$ consists solely of bottom streaming (see Eqn. 21 ), which is provided as a vertically distributed function (see Eqn. 27). The geopotential function $\varphi^{c}$ contains both pressure gradient and the effect of wave shoaling (see Eqn. 13). Finally, surface streaming (Eqn. 28 ) is provided as a surface boundary condition. Unlike Lentz et al. (2008), we do not impose a noflow boundary condition at the sea bed, but provide a bottom stress as logarithmic bottom drag with a roughness length of $0.001 \mathrm{~m}$.

The model domain is horizontally uniform with a constant water depth of $12 \mathrm{~m}$. The domain is doubly periodic, with cross-shore and longshore widths of $40 \mathrm{~m}$ each and a grid resolution of $10 \mathrm{~m}$ in both horizontal directions. Vertically the domain is distributed into 150 layers with enhanced resolution of less than $1 \mathrm{~cm}$ close to bottom and surface boundaries. High resolution is necessary to correctly depict the bottom streaming induced forcing (Eqn. 27). Horizontally uniform wave forcing in the form of wave height, period, direction and length is provided. Instead of providing a wall at the inshore boundary, a vertically uniform body force is imposed in the direction opposite to that of wave propagation with strength such that the net Lagrangian mean flow is zero, as in Lentz et al. (2008). This body force emulates the effect of wave shoaling and pressure gradient. Finally, vertical viscosity values $\left(K_{M}\right)$ are vertically uniform and prescribed for a range of values varying from $10^{-6}$ to $10^{0} \mathrm{~m}^{2} \cdot \mathrm{s}^{-1}$.

\subsubsection{Effect of vertical viscosity}

The first simulation examines the effect of vertical viscosity on the shape of undertow profiles which are shown in Fig. 17a. Inshore propagating, normally incident waves with a significant wave height of $2 \mathrm{~m}$ and period $7 \mathrm{~s}$ are prescribed over the model domain. $K_{M}$ values are varied from $10^{-6}$ to $10^{0} \mathrm{~m}^{2} \cdot \mathrm{s}^{-1}$. The results show that when $K_{M}$ takes values between $10^{-6}$ and $10^{-4} \mathrm{~m}^{2} \cdot \mathrm{s}^{-1}$, the undertow profile has a convex shape with weak offshore/inshore flow at the bottom boundary layer and stronger offshore flow at the surface. For larger $K_{M}$ values $\left(10^{-3}-10^{-2}\right.$ $\left.\mathrm{m}^{2} \cdot \mathrm{s}^{-1}\right)$, the shape of the undertow profile becomes concave consisting of inshore flow at the bottom layer and stronger offshore directed flow at the upper half of the water column. Closer to the sea surface, the velocity magnitude either remains constant or reduces slightly. For even larger $K_{M}$ values $\left(10^{-1}-10^{0} \mathrm{~m}^{2} \cdot \mathrm{s}^{-1}\right)$ the cross-shore velocity profile becomes parabolic in shape with maximum offshore flow at the middle of the water column and slightly reduced flows at the surface and bottom layers. These vertical profiles are similar to those obtained by Lentz et al. (2008).

Longshore velocity profiles for the different vertical viscosity values used are shown in Fig. 17b. For $K_{M}$ values between $10^{-6}$ and $10^{-5} \mathrm{~m}^{2} \cdot \mathrm{s}^{-1}$, the longshore flows are vertically uniform over the majority of the water column with reduced velocities near the sea surface and bed, while stronger vertical shear is observed for $K_{M}$ values between $10^{-4}$ and $10^{-3} \mathrm{~m}^{2} \cdot \mathrm{s}^{-1}$. These profiles become vertically uniform and negligibly small for $K_{M}$ values greater than $10^{-3} \mathrm{~m}^{2} \cdot \mathrm{s}^{-1}$ (see Fig. 17b).

The observed changes in cross-shore and longshore vertical profiles as a function of viscosity can be explained on the basis of Eqns. 11 and 12 . For low $K_{M}$ values and assuming normally incident waves, Eqns. 11 and 12 can be simplified to: 


$$
\begin{aligned}
& \underbrace{-H_{z}\left(\frac{f v}{m n}\right) \cong-\left.\frac{H_{z}}{n} \frac{\partial \varphi^{c}}{\partial \xi}\right|_{z}}+\underbrace{\frac{H_{z} \mathcal{F}^{w \xi}}{m n}} \\
& \mathrm{COR} \mathrm{PG} \mathrm{BtSt} \\
& H_{z}\left(\frac{f u}{m n}\right)+H_{z}\left(\frac{f u^{s t}}{m n}\right) \cong 0 \\
& \text { COR StCOR }
\end{aligned}
$$

Eqn. 51 suggests that for cross-shore flows, the higher order momentum balance occurs between the Hasselmann stress $\left(f u^{s t}, f v^{s t}\right)$ and Coriolis force, which creates the observed convex profile for cross-shore flows similar to the shape of Stokes drift. The longshore flow is contingent upon pressure gradient (vertically uniform) followed by bottom and surface streaming contribution due to veering (Lentz et al. 2008). This leads to a vertically uniform longshore flow (Fig. 17b) with slightly reduced velocities near the surface and bottom layers.

For higher $\mathrm{K}_{\mathrm{M}}$ values the contributions of Coriolis force and Hasselmann stress are negligible so that Eqn. 11 can be written as:

$$
0 \cong \frac{-\left.\frac{H_{z}}{n} \frac{\partial \varphi^{c}}{\partial \xi}\right|_{z}}{\mathrm{PG}}+\frac{\frac{H_{z} \mathcal{F}^{w \xi}}{m n}}{\mathrm{BtSt}}+\frac{\frac{\partial}{\partial s}\left(K_{M} \frac{\partial u}{\partial s}\right)}{\mathrm{VM}}
$$

If bottom streaming is provided as a bottom boundary condition and surface streaming as a surface stress (see Lentz et al., 2008), Eqn. 52 takes a quadratic form and can be solved analytically for cross-shore flows. In such case, the vertical profile of cross-shore velocity is parabolic in shape with maximum flow at the center (see Fig. 3 in Lentz et al., 2008). Numerical solution of Eqns. 11 and 12 generate a similar in shape profile (Fig. 17a) but with smaller curvature since the flows at the surface and bottom are stronger than those obtained by the analytical solution.

\subsubsection{Effect of wave height}

In a second set of simulations, the effect of wave height on cross-shore and longshore velocity profiles is examined. These simulated velocity profiles are compared to the data presented in Lentz et al (2008) that represent velocity profiles averaged over a variety of wave heights corresponding to times of minimal wind forcing

The average significant wave height conditions at MVCO are $1.0 \mathrm{~m}$, with a standard deviation of $0.5 \mathrm{~m}$, and peak wave period varying between 4 and $7 \mathrm{~s}$ (Lentz et al., 2008). We chose a set of simulations with a constant $K_{M}=10^{-5} \mathrm{~m}^{2} \cdot \mathrm{s}^{-1}$ and a normally incident wave height with values from 0 to $3.5 \mathrm{~m}$ with an interval of $0.25 \mathrm{~m}$ and a peak period of $7 \mathrm{~s}$. Velocity profiles obtained for each wave height value were grouped together into four groups corresponding to wave height intervals of $0-0.75 \mathrm{~m}, 0.75-1.5 \mathrm{~m}, 1.5-2.25 \mathrm{~m}$ and $2.25-3.5 \mathrm{~m}$ and subsequently averaged. These averaged profiles are shown in Fig. 18 together with the published data of Lentz et al. (2008). Since a $\operatorname{low}^{3} \mathrm{~K}_{\mathrm{M}}$ value was used, the undertow profiles have a convex shape similar

\footnotetext{
${ }^{3}$ In the absence of wind forcing and since the flows discussed here are weak, a low $\mathrm{K}_{\mathrm{M}}$ value of $10^{-5} \mathrm{~m}^{2} \mathrm{~s}^{-1}$ provides best agreement to field observations. Use of a GLS mixing scheme will result in a parabolic $\mathrm{K}_{\mathrm{M}}$ with maximum value $\left(\sim 10^{-3}\right)$ at the middle of the water column. The vertical profile of cross-shore velocity will have a parabolic shape similar to the green and black velocity profiles shown in Fig. 17a.
} 
to that of the observations. For waves corresponding to the first three groups, model results closely agree with the reported cross-shore velocity profiles of Lentz et al (2008). These profiles have a weak inshore flow at the bottom boundary and offshore directed flow within rest of the water column (Fig. 18a). The decrease in the observed velocities near the surface may be due to measurement errors (i.e., contamination of top bin by surface reflections from the sidelobes of the ADCP acoustic pulses during large waves because of reduced water depth in the wave troughs (Lentz et al., 2008). For the group 2.25-3.25 m, the model predicts undertow flows that are higher than the observed ones. The rms error analysis (normalized by maximum observed data, Table 6) show small errors for first three groups (21-34\%), and higher errors for the last group (48\%). Longshore velocity is negligible for small waves $(0-0.75 \mathrm{~m})$, and of the order 1-2 $\mathrm{cm} \cdot \mathrm{s}^{-1}$ for the second and third wave group. The simulated profiles show similar magnitudes for the first three wave groups, though the vertical structure is slightly different. The model simulated longshore flow for waves $\approx 2.25-3.50 \mathrm{~m}$ shows similar shape, but weaker flows than the observed ones (Fig. 18b). RMS errors in simulated longshore velocities for first three groups are approximately 50\%, while for the last group it is $83 \%$ (Table 6). The overall errors in crossshore and longshore velocities are $11 \%$ and $29 \%$, respectively.

(a) $\mathrm{u}\left(\mathrm{m} \cdot \mathrm{s}^{-1}\right)$

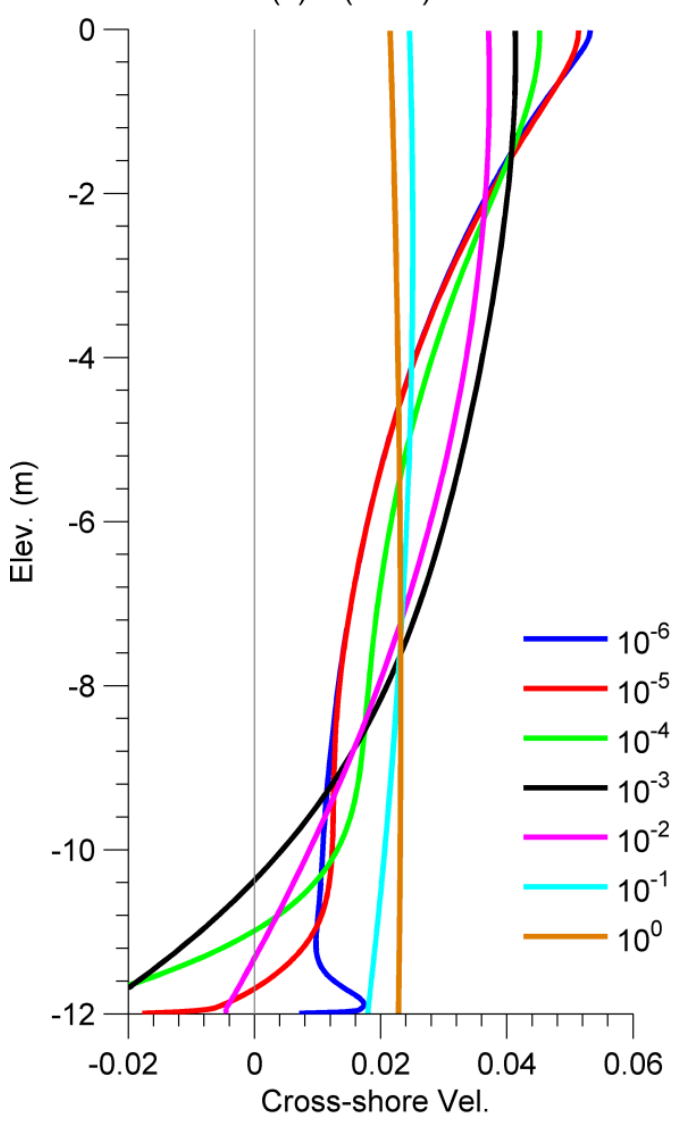

(b) $\vee\left(m \cdot s^{-1}\right)$

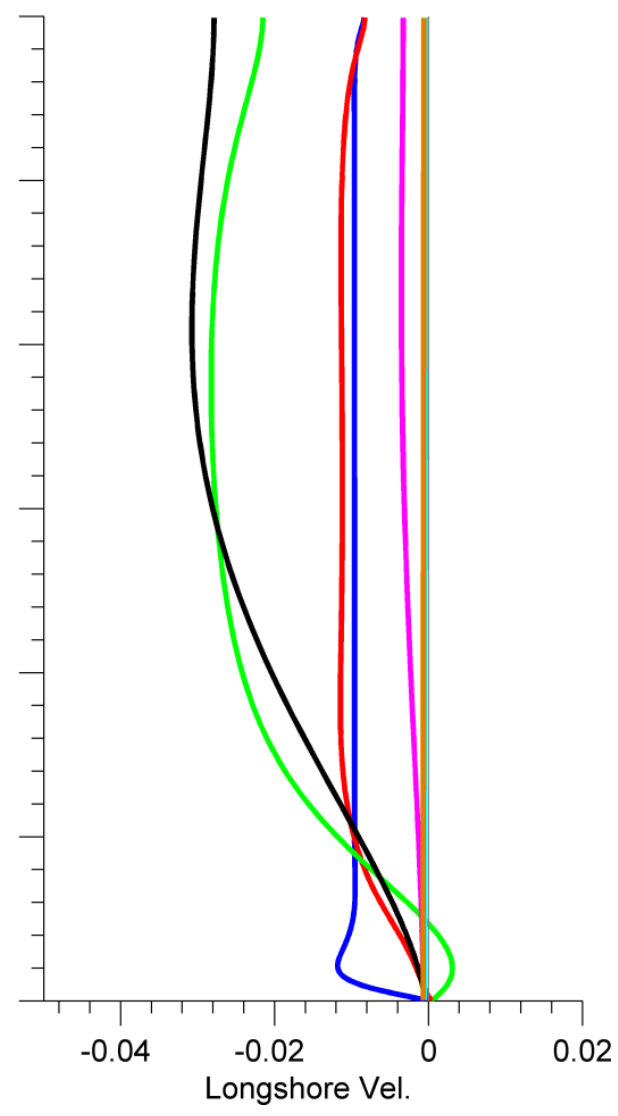

Figure 17. Cross-shore (a) and longshore (b) velocity profiles from model simulations with constant vertical viscosity $\left(K_{M}\right)$ values ranging from $10^{-6}$ to $10^{0} \mathrm{~m}^{2} \mathrm{~s}^{-1}$. The model simulations were carried out assuming a normally incident wave with significant wave height of $2 \mathrm{~m}$ and wave period of $7 \mathrm{~s}$. 
It is important to note that the observations of Lentz et al (2008) are averaged over varying wave heights and periods, while the model simulations were carried out for a particular set of wave heights and a single period. Furthermore, small differences may also occur due seasonal influences on the velocity profiles (see Lentz et al., 2008). Overall, the observed and simulated cross-shore velocity profiles show similar shapes and magnitude suggesting that the model as implemented in this work can successfully simulate inner-shelf flows under the forcing of waves.

(a) $u\left(m \cdot s^{-1}\right)$

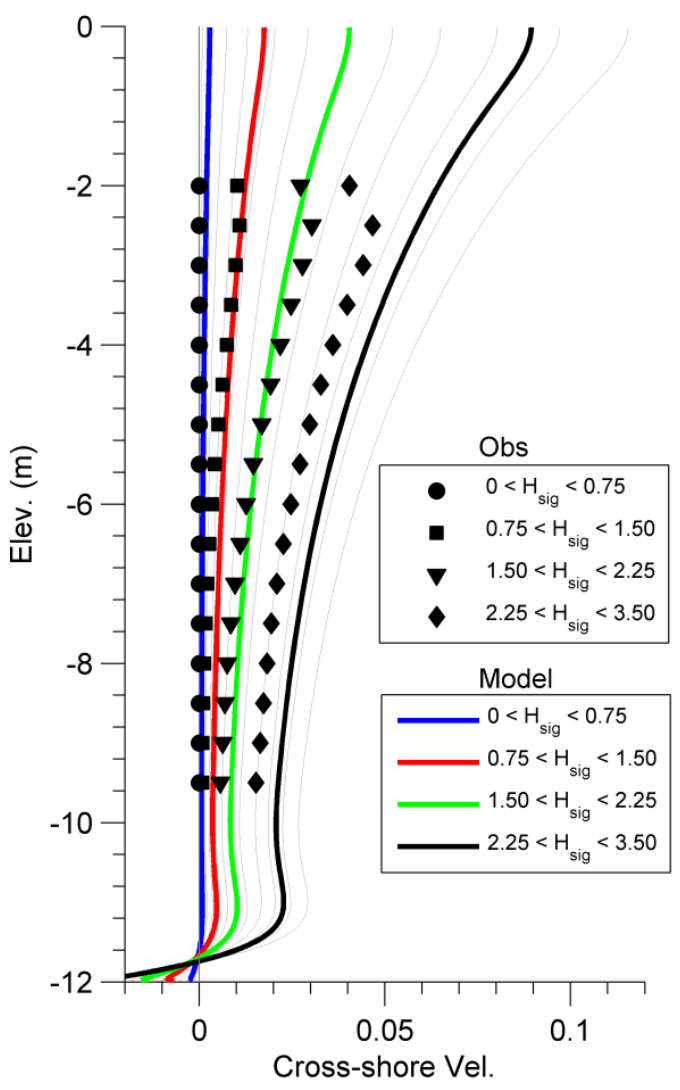

(b) $v\left(m \cdot s^{-1}\right)$

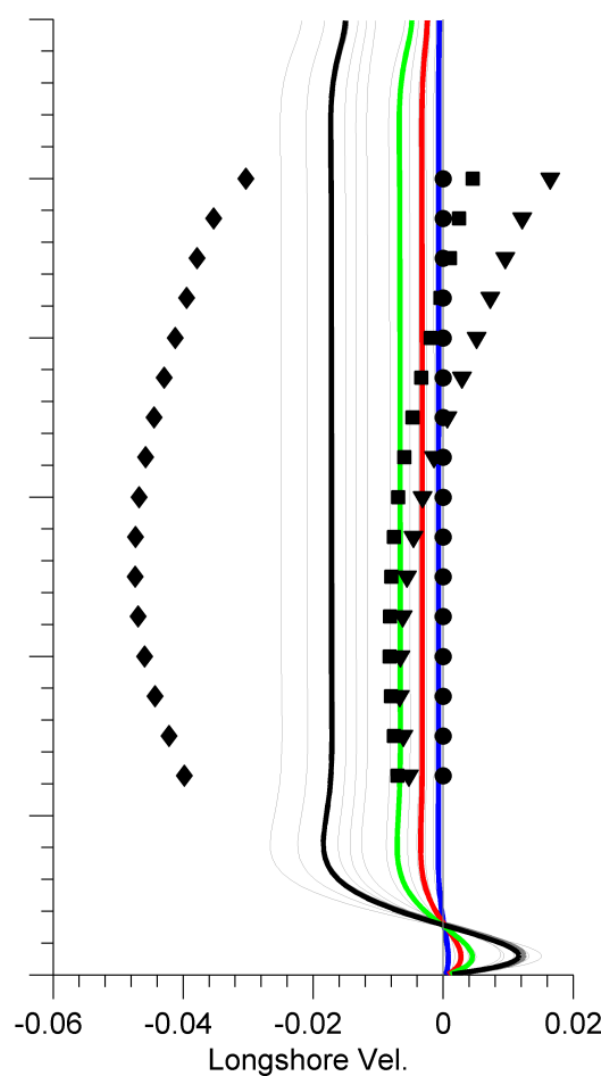

Figure 18. Observed (from Lentz et al, 2008) and simulated cross-shore (a) and longshore (b) velocity profiles for different ranges of significant wave height $\left(\mathrm{H}_{\mathrm{sig}}\right)$. Individual model profiles estimates for wave height values from 0 to $3.5 \mathrm{~m}$ with an interval of $0.25 \mathrm{~m}$ are shown as thin grey lines while the thicker solid lines show velocity profiles averaged over specific wave height ranges as shown in insert. Simulations were carried out with a constant viscosity of $10^{-5} \mathrm{~m}^{2} \mathrm{~s}^{-1}$.

\section{Discussion}

VF and RS representations are two different approaches used to incorporate the effects of surface gravity waves on the mean flow. The VF representation treats the conservative (vortex force, Bernoulli head and quasi-static pressure gradient) and non-conservative processes (breaking acceleration etc.) separately. On the other hand, the RS based approach accommodates wave-averaged effects through the gradient of the radiation stress tensor term. The differences between the two approaches are discussed using simulation results from models based on either 
representation. In particular, velocity and sea-surface elevation simulated results are first compared, followed by an analysis of momentum balance results.

Table 6. The RMS error (normalized by the maximum value) for the simulated cross-shore and longshore velocities for wave-induced flows in the inner shelf ( $\sec 4.4)$.

\begin{tabular}{|c|c|c|}
\hline \multirow{2}{*}{$\begin{array}{c}\text { Wave Height } \\
\text { Group }\end{array}$} & \multicolumn{2}{|c|}{ RMS Error (\%) } \\
\cline { 2 - 3 } & $\begin{array}{c}\text { Cross-shore } \\
\text { Vel. }\end{array}$ & $\begin{array}{c}\text { Longshore } \\
\text { Vel. }\end{array}$ \\
\hline $0<H_{\text {sig }}<0.75$ & NA & NA \\
\hline $0.75<H_{\text {sig }}<1.5$ & 21.4 & 52.0 \\
\hline $1.5<H_{s i g}<2.25$ & 34.3 & 48.9 \\
\hline $2.25<H_{\text {sig }}<3.50$ & 48.4 & 83.2 \\
\hline Overall & 11.3 & 29.5 \\
\hline
\end{tabular}

All sea surface height $\left(\zeta^{c}\right)$ simulations (i.e., VF, $\mathrm{RS}_{2 \mathrm{D}}$ and Eqn. 48), for the planar beach case, show a similar cross-shore structure (see Fig. 1b). Also, the Eulerian mean cross-shore velocity (Fig. $2 \mathrm{~g}$ ) is identical for all simulations. Longshore velocity ( $\overline{\mathrm{v}}$, Fig. $2 \mathrm{~h}$ ) from $\mathrm{RS}_{2 \mathrm{D}}$ is strongest at $x \sim 300 \mathrm{~m}$, which also corresponds to the location of maximum $\overline{\mathrm{v}}$ derived from the analytical solution (Eqn. 48); however this location is slightly offshore that of maximum $\bar{v}$ estimated using the VF approach. The overall $\bar{v}$ cross-shore structure slightly differs between the methods, with the analytical solution giving the largest gradients around the maximum point. This difference is mainly due to horizontal viscous mixing in the $\mathrm{RS}_{2 \mathrm{D}}$ solution while for the VF approach this is attributed to horizontal advection and vortex force (see below discussion on momentum balance).

In the barred beach simulations (see Sec. 4.2), $\zeta^{c}$ from $\mathrm{RS}_{2 \mathrm{D}}$ (Run 1) and VF (Run 2, no wave rollers/wave mixing) show differences at the bar-crest location and further inshore at the shoreward boundary. These locations correspond to areas with high velocity shear in the crossshore current profiles (Fig. 9a). At these locations the Bernoulli head (Eqn. 5) contribution becomes important (see Fig. 11c) and modifies the total pressure gradient force, which in turn modifies the $\zeta^{c}$ values. It is important to point out that the radiation stress divergence term is of the same order as the quasi-static pressure gradient (see cross-shore momentum balance) and it cannot resolve wave-averaged effects like the Bernoulli head (see Lane et al., 2007).

The Eulerian, depth-averaged longshore simulated velocities (Fig. 5d) using $\mathrm{RS}_{2 \mathrm{D}}$ and VF (with no rollers, i.e., $\alpha_{r}=0$, Run 2 ) differ significantly in terms of cross-shore structure, maximum velocity and location of peak flow. These differences are better explained by the cross-shore variation of the depth-averaged longshore momentum balance terms.

The depth integrated momentum balance for a $\mathrm{RS}_{2 \mathrm{D}}$ based implementation is (see Warner et al., 2008a): 


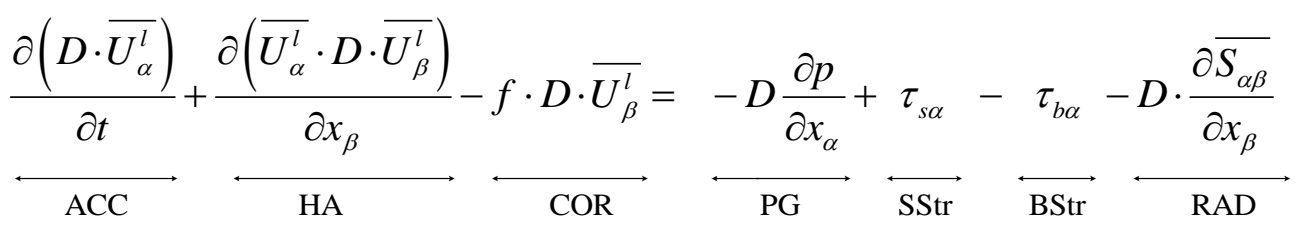

where, the subscripts $\alpha$ and $\beta$ represent the horizontal coordinates, the superscript $l$ denotes Lagrangian mean flows, and the overbar indicates depth averaged values. $\overline{U_{\alpha}^{l}}$ and $\overline{U_{\beta}^{l}}$ are depthaveraged Lagrangian mean velocities; $D$ is the total water depth, $f$ is the Coriolis parameter, $p$ is the total barotropic pressure, $\tau_{s \alpha}$ and $\tau_{s \beta}$ are surface and bottom stresses, respectively, and $\overline{S_{\alpha \beta}}$ is the depth-averaged radiation stress.

In a similar manner, simplified equations for the VF approach can be obtained from Eqns. 11 and 12 after removing the curvilinear terms, body forces, horizontal and vertical mixing and using Cartesian coordinates. Adding together the Coriolis and Stokes-Coriolis forces, moving the horizontal vortex force to the left hand side of the equation and vertically averaging, the VF simplified momentum balance equation becomes:

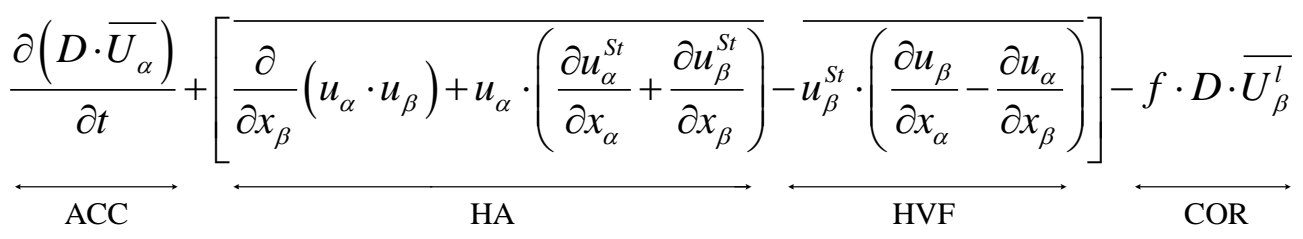

$$
=\frac{-\left.D \frac{\partial \overline{\varphi^{c}}}{\partial x_{\alpha}}\right|_{z}}{\mathrm{PG}_{\mathrm{SStr}}} \underset{\mathrm{BStr}}{\stackrel{\mathrm{BA}}{\longrightarrow}}
$$

where, $\overline{U_{\alpha}}$ is the depth-averaged Eulerian mean velocity; $u_{\alpha}$ and $u_{\beta}$ are the three-dimensional Eulerian mean velocities; $u_{\alpha}^{S t}$ is the three-dimensional Stokes velocity; $\overline{\varphi^{c}}$ is the verticallyintegrated geopotential function (Eqn. 13), and $\overline{\mathcal{F}^{w \xi}}$ is the vertically integrated non-conservative wave forcing. It is important to note that the VF based model solves for three-dimensional flows, and the Eulerian mean and Stokes velocity based advective accelerations in Eqn. $54\left(2^{\text {nd }}\right.$ term in left hand side) are vertically-averaged from their three-dimensional distribution. This is not the case for Lagrangian advection ( $2^{\text {nd }}$ term in left hand side of Eqn. 53) in the $\mathrm{RS}_{2 \mathrm{D}}$ based model, where the term is obtained as a function of depth-averaged Lagrangian mean flows.

For the planar beach case (see section 4.1), the vertically-integrated cross-shore momentum balance in the cross-shore direction using VF suggests a balance between PG and $\mathrm{BA}$, while the $\mathrm{RS}_{2 \mathrm{D}}$ results show a balance between PG and divergence of radiation stress (RAD) (Eqns. 53 and 54) (Figs. 19a and 19b). In the shoaling region $(800 \mathrm{~m}>x>500 \mathrm{~m})$ for the VF approach, the quasi-static pressure gradient ( $P_{q s x}$, Eqns. 7 and 13), a wave-induced effect, balances the Eulerian pressure response ( $P_{c x}$, Eqn. 49, see Fig. 4 c), which leads to a wave setdown. The total pressure gradient which is calculated as the sum of the individual components (Eqn. 49) therefore becomes zero only in the shoaling region (i.e., $x>500 \mathrm{~m}$, see Fig. 19a). On the other hand, in the $\mathrm{RS}_{2 \mathrm{D}}$ approach the wave-averaged effect is represented only by the RAD 
term (Fig. 19b) and it is balanced by the cross-shore pressure gradient (PG, only Eulerian pressure response, see Eqn. 53; Fig. 19c). As the wave energy density changes (i.e., increase during wave shoaling and decrease during wave breaking in the surf zone) the gradient of radiation stress follows these changes obtaining positive values in the shoaling region (i.e., $x>$ $500 \mathrm{~m}$, Fig. 18b) which change to negative values inside the surf zone. The positive contribution of radiation stress gradient is balanced by a negative pressure gradient forcing (Fig. 19b) and therefore it can be considered representing the forces equivalent to the quasi-static pressure gradient $\left(P_{q s x}\right.$, Fig. $\left.4 \mathrm{c}\right)$ in the VF representation.
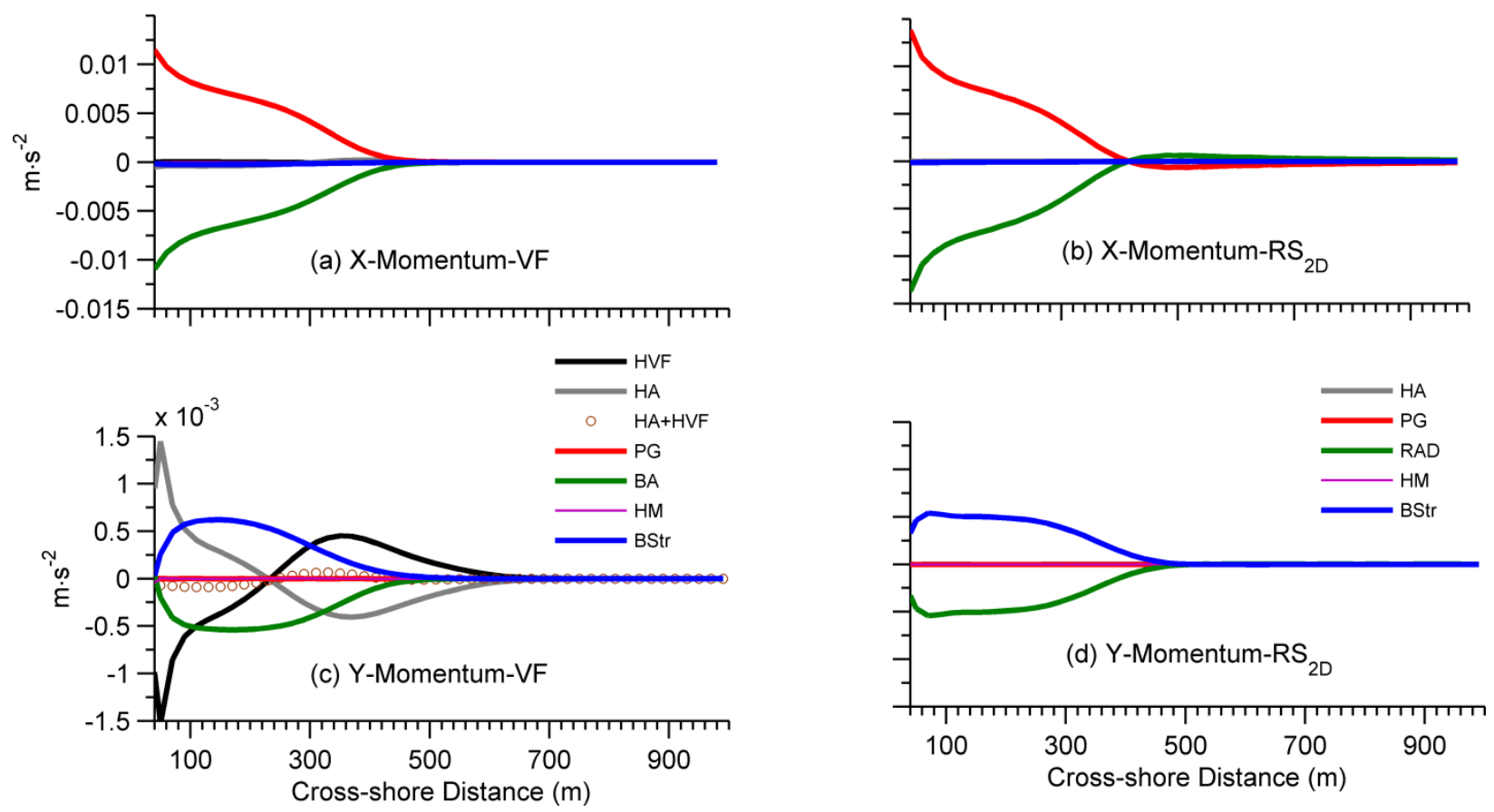

Figure 19. Cross-shore distribution of cross-shore ( $a, b)$ and longshore (c, $d)$ momentum balance terms from the VF model ( $\mathrm{a}, \mathrm{c}$ ) and $\mathrm{RS}_{2 \mathrm{~d}}(\mathrm{~b}, \mathrm{~d})$ model simulations for obliquely incident waves on a planar beach (Sec 4.1).

The cross-shore distribution of the longshore momentum balance terms BStr and BA in the VF approach are similar and almost mirror images of each other (Fig. 19c). A similar relation is found for the HA and HVF terms although both of them change signs at the location of maximum longshore velocity. However, HA and HVF do not add to exactly zero, with a positive sum seaward of point of maximum $v$ and a negative sum landward of maximum $v$ (Fig. 19c) These differences in the cross-shore distribution of HVF and HA leads to a decrease in BStr seaward of maximum $v$ and an increase landward of maximum $v$, and a corresponding landward shift of the cross-shore profile of longshore velocity. Though the imbalance of HVF and HA may seem to play a minor role in here, in the barred beach case these advective accelerations are important as they modify the cross-shore structure of longshore momentum balance (see below). The $\mathrm{RS}_{2 \mathrm{D}}$ model implements Lagrangian averaging and a part of vortex force is accommodated in the HA term $\left(2^{\text {nd }}\right.$ term in Eqn. 53); thus the only balance observed in this case is between BStr and RAD (Fig. 19d). 
For the barred beach case (see section 4.2) the cross-shore momentum balance terms outside the surf zone are zero for both VF and $\mathrm{RS}_{2 \mathrm{D}}$ approaches (Figs. 20a and b) as no shoaling takes place in this case (see Fig. 5a). Within the surf zone, the major balance is between PG and $\mathrm{BA}$ for the VF approach and PG and RAD, for the $\mathrm{RS}_{2 \mathrm{D}}$ approach.
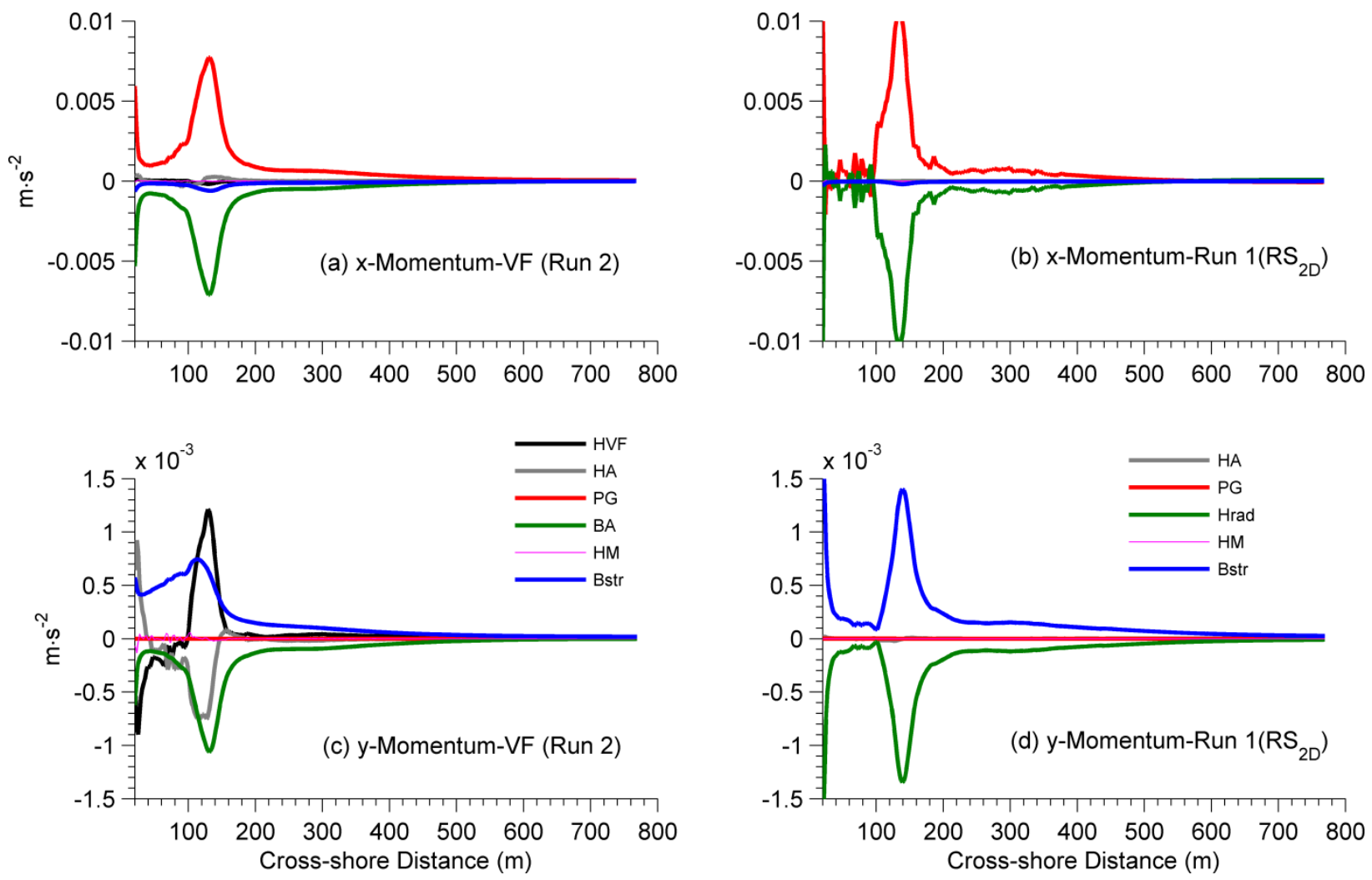

Figure 20. Cross-shore distribution of cross-shore (a, b) and longshore (c, d) momentum balance terms from the VF (a, c) and $\mathrm{RS}_{2 \mathrm{~d}}$ (b, d) models for obliquely incident spectral waves on a barred beach (Sec. 4.2). The VF and $\mathrm{RS}_{2 \mathrm{D}}$ simulation corresponds to Run 6 (VF model with wave rollers, $\alpha_{r}=1$ and no wave mixing) and Run 2 (radiation stress model) as described in Table 3.

The VF and $\mathrm{RS}_{2 \mathrm{D}}$ models suggest major difference in the contribution of dominant terms in the longshore momentum balance for the barred beach. The HVF and HA terms have similar cross-shore structure but different magnitude in the surf zone (Fig. 20c). The HVF term becomes significant in the vicinity of the bar, and it has the same structure and order of magnitude (but of opposite sign) as the BA term. On the contrary, the $\mathrm{RS}_{2 \mathrm{D}}$ model still suggests a balance between BStr and RAD (Fig. 20d) and zero contribution from HA because the depth-averaged Stokes and anti-Stokes flow (Eulerian mean flow) are of opposite sign and same cross-shore structure. This makes the Lagrangian mean flow $\left(\overline{U_{\alpha}^{l}}\right)$ zero in Eqn. 53, leading to no contribution of HA. This is not the case for the vertically-integrated terms from the VF simulations. In there, although the vertically-averaged Stokes and Eulerian mean cross-shore flows balance each other (i.e., $\overline{u_{\alpha}^{S t}}=-\overline{u_{\alpha}}$ ), their vertical structure are significantly different (e.g., see Figs. 2 and 9). This difference in structure creates an inequality in the contribution of vertically integrated horizontal advection and vortex-forces (i.e., assuming alongshore uniformity in Eqn. 54, 
$\overline{\frac{\partial\left(u_{\alpha} \cdot u_{\beta}\right)}{\partial x_{\beta}}} \neq \overline{u_{\beta}^{S t}\left(\frac{\partial u_{\beta}}{\partial x_{\alpha}}\right)}+\overline{u_{\beta}\left(\frac{\partial u_{\beta}^{S t}}{\partial x_{\beta}}\right)}$, see Fig. 20c), which in the present case manifests itself in the form of an inshore shift of the whole longshore flow pattern.

\section{Summary and Conclusions}

The wave-current coupling component of the three-dimensional circulation model ROMS (a public domain model) has been updated with the vortex force formalism presented by MRL04 and U10 and enhanced with a GLS mixing scheme. The modeling system has been applied to study four test cases including littoral velocities in a synthetic planar and a natural, barred beach due to obliquely incident waves, complex flow fields in a synthetic barred beach with rip channels, and validated against undertow profiles in an inner-shelf setting.

The model results for a planar beach show qualitative agreement to laboratory results and field measurements. Simulations for the DUCK' 94 experiment provided close agreement to measured cross-shore and longshore velocity profiles by Garcez-Faria et al. (1998, 2000). Normalized rms error analysis suggests that nonlinear processes like wave rollers and waveinduced mixing are important. Recent studies by Ribas et al. (2011) show that the wave rollers can be important in the evolution of crescentic bars. In this study we used constant values for $\alpha_{r}$ and $c_{\varepsilon \omega}$ to account for the portion of energy responsible for creation of roller energy, and to identify the portion of wave dissipation responsible for turbulent mixing. It has been shown (Apotsos et al., 2007) that both these processes can be influenced by local beach profile, water depth, wave height, percentage of wave dissipation etc. It is recommended that instead of using constant values for $\alpha_{r}$ and $c_{\varepsilon \omega}$ over the entire surf zone, spatially varying non-dimensional quantities should be adopted. These should be a function of local cross-shore position and instantaneous wave parameters (see Cambazoglu and Haas, 2011).

Momentum balance analysis shows a primary higher order balance between quasi-static pressure gradient and breaking acceleration in the cross-shore direction, while in the longshore a balance is achieved between bottom stress, breaking acceleration, horizontal advection and horizontal vortex forces. The contribution of vortex force has not been explicitly identified in studies based on radiation stress approach, but results from a depth-averaged, Lagrangian, radiation stress based model (Warner et al., 2008a) suggest that the effect of the vortex force term is implicitly included within the horizontal advection. It is also important to note that when the vertical structure of Stokes and Eulerian mean velocity are different, the contribution of vortex force is not completely balanced by horizontal advection and this can change the magnitude and cross-shore location of longshore velocity, as is observed for the DUCK' 94 simulations.

The simulation for nearshore barred morphology with rip channels clearly demonstrates the ability of the model to reproduce the circulation patterns that have been observed in laboratory studies (Haller et al., 2002; Haas and Svendsen, 2002). Furthermore, within and outside the rip channel area, flow vorticity interacts with the wave-induced Stokes drift leading to strong alongshore contribution of vortex force, which is balanced by the pressure gradient term.

The simulated profiles of undertow for conditions outside the surf zone closely agree with measured flows at MVCO. Furthermore, the shape of the profiles varies as a function of vertical viscosity as suggested by Lentz et al. (2008). 
Overall, the method of including the surface gravity waves through VF formalism leads to a clear separation of conservative and non-conservative contribution in the momentum balance equations. The conservative processes are important outside the surf zone, while within the surf zone wave breaking induced flows dominate the momentum balance. This delineation of conservative and non-conservative wave forcing allows implementation of the VF formalism as a tool to evaluate flow fields both within inner shelf and surf zone environments. This application is a significant step in three-dimensional modeling of wave driven flows providing an alternative to models based on the RS approach (see Wang and Chen, 2010; Sheng and Liu, 2011; Kumar et al., 2011a). The VF representation as presented in this paper has been used to study wave-current interaction in a tidal-inlet along with validation against measurements (Olabarrieta et al., 2011) and simulations under a variety of environments are underway.

\section{Acknowledgements}

The first two authors were supported by a NOAA/IOOS Grant (Integration of Coastal Observations and Assets in the Carolinas in Support of Regional Coastal Ocean Observation System Development in the Southeast Atlantic) and a cooperative agreement between U.S. Geological Survey and University of South Carolina as part of the Carolinas Coastal Change Processes Project. Also G. Voulgaris was partially supported by the National Science Foundation (Awards: OCE-0451989 and OCE-0535893). We would like to thank Dr. Lentz for his comments regarding the inner shelf test case. Finally, we would also like to thank the ROMS and SWAN code developers for their hard work in developing this open source code, and the staff and personnel involved in collecting and maintaining the DUCK'94 experiment dataset.

Computational support was provided by the Research Computing Center and the Center for Computational Research and CyberInfrastructure Support at University of South Carolina.

\section{List of References}

Aagaard, T., Greenwood, B., Nielsen, J., 1997. Mean currents and sediment transport in a rip channel, Marine Geology, Volume 140, 1-2, 25-45.

Agrawal, Y.C., Terray, E.A., Donelan, M.A., Hwang, P.A., Williams, A.J., Drennan, M., Kahma, K.K., Kitaigorodskii, S.A., 1992. Nature, 359, 219-220.

Apotsos, A., Raubenheimer, B., Elgar, S., Guza, R.T., Smith, J.A.., 2007. Effect of wave rollers and bottom stress on wave setup. Journal of Geophysical Research, 112, C02003, doi: 10.1029/2006JC003549 Andrews, D.G., McIntyre, M.E., 1978a. An exact theory of nonlinear waves on a Lagrangian-mean flow. Journal of Fluid Mechanics, 89, 609-646.

Andrews, D.G., McIntyre, M.E., 1978b. On wave-action and its relatives. Journal of Fluid Mechanics, 89, 647-664.

Ardhuin, F., Rascle, N, Belibassakis, K.A., 2008. Explicit wave-averaged primitive equations using a generalized Lagrangian mean. Ocean Modeling, 20, 35-60.

Ardhuin, F., Rascle, N, Belibassakis, K.A., 2008b. Comments on "The three-dimensional current and surface wave equations” J. Phys. Oceanogr., 38, pp. 1340-1350. 
Battjes, J.A., Janssen, J.P.F.M., 1978. Energy loss and set-up due to breaking of random waves. Proceedings of 16th International Conference Coastal Engineering, ASCE, pp. 569-587.

Bennis, A., Ardhuin, F., Dumas., F., 2011. On the coupling of wave and three-dimensional circulation models: Choice of theoretical framework, practical implementation and adiabatic tests. Ocean Modeling, 40, 260-277.

Carniel S., Warner J.C., Chiggiato, J., Sclavo, M., 2009. Investigating the impact of surface wave breaking on modelling the trajectories of drifters in the Northern Adriatic Sea during a wind-storm event. Ocean Modeling, 30, pp. 225-239. DOI:10.1016/j.ocemod.2009.07.001.

Cambazoglu, M.K., Haas, K.A., 2011. Numerical modeling of breaking waves and cross-shore currents on a barred beach. Journal of Waterway, Port, Coastal and Ocean Engineering. doi:10.1061/(ASCE)WW.1943-5460.0000096

Church, J. C., Thornton, E.B., 1993. Effects of breaking wave induced turbulence within a longshore current model. Coastal Engineering, 20, 1-28.

Collins, J.I., 1972: Prediction of shallow water spectra, Journal of Geophysical Research, 77, No. 15, 2693-2707.

Craik, A.D.D., Leibovich, S., 1976. A rational model for Langmuir circulations. Journal of Fluid Mechanics, 73, 401-426.

Craig, P. D., Banner, M. L., 1994. Modeling wave-enhanced turbulence in the ocean surface layer. Journal of Physical Oceanography, 24, 2546-2559.

Durski, S.M., Glenn, S.M., Haidvogel, D., 2004. Vertical mixing schemes in the coastal ocean: Comparison of the level 2.5 Mellor-Yamada scheme with an enhanced version of the K profile parameterization. Journal of Geophysical Research, 109, C01015.

Eldeberky, Y., Battjes, J.A., 1996: Spectral modelling of wave breaking: Application to Boussinesq equations, Journal of Geophysical Research, 101, No. C1, 1253-1264

Elgar, S., Guza, R.T., Raubenheimer, B., Herbers, T.H.C., Gallagher, E.L., 1997. Spectral evolution of shoaling and breaking waves on a barred beach. Journal of Geophysical Research, 103, 15797-15805.

Feddersen, F., Guza, R. T., Elgar, S., Herbers, T. H. C., 1998. Alongshore momentum balances in the nearshore. Journal of Geophysical Research, 103, 15,667-15,676.

Feddersen, F., Trowbridge, J. H., 2005. The effect of wave breaking on surfzone turbulence and alongshore currents: a modeling study. Journal of Physical Oceanography, 35, 2187-2203.

Flather, R. A., 1976: A tidal model of the northwest European continental shelf. Mern. Soc. Roy. Sci. Liege, Ser. 6, 10, 141-164.

Gallagher, E.L., Elgar, S., Guza, R.T., 1998. Observations of sand bar evolution on a natural beach. J. Geophys. Res. 103, 3203-3215.

Garcez Faria, A.F., Thornton, E.B., Stanton, T.P., Soares, C.V., Lippmann, T.C., 1998. Vertical profiles of longshore currents and related bed stress and bottom roughness. Journal of Geophysical Research, 103, $15667-15676$. 
Garcez-Faria, A. F., Thornton, E. B., Lippmann, T. C., Stanton, T. P., 2000. Undertow over a barred beach. Journal of Geophysical Research, 105, 16,999-17,010.

Garrett, C., 1976: Generation of Langmuir circulations by surface waves-A feedback mechanism. Journal of Marine Research, 34, 117-130.

Grant, S. B., Kim, J. H., Jones, B. H., Jenkins, S. A., Wasyl, J., Cudaback, C., 2005. Surf zone entrainment, along-shore transport, and human health implications of pollution from tidal outlets. J. Geophys. Res., 110, C10025, doi:10.1029/2004JC002401.

Groeneweg, J., Klopman, G., 1998. Changes in the mean velocity profiles in the combined wave-current motion described in GLM formulation. Journal of Fluid Mechanics, 370, 271-296.

Haas, K. A., Svendsen, I. A., 2002. Laboratory measurements of the vertical structure of rip currents. Journal of Geophysical Research, 107(C5), 3047, doi: 10.1029/2001JC000911.

Haas, K.A., Svendsen, I.A., Haller, M.C., Zhao, G., 2003. Quasi-three dimensional modeling of rip current system. J. Geophys. Res., 108.

Haas, K. A., Warner, J.C., 2009. Comparing a quasi-3D to a full 3D nearshore circulation model: 1035 SHORECIRC and ROMS. Ocean Modeling. 26, 91-103.

Haidvogel, D.B., Arango, H.G., Hedstrom, K., Beckmann, A., Malanotte-Rizzoli, P., Shchepetkin, A.F., 2000. Model Evaluation Experiments in the North Atlantic Basin: Simulations in Nonlinear TerrainFollowing Coordinates, Dynamics of Atmosphere and Oceans, 17, 32, 239-281.

Haidvogel, D. B. , Arango, H.G., Budgell, W. P., Cornuelle, B. D., Curchitser, E., Di Lorenzo, E., Fennel, K., Geyer, W. R., Hermann, A. J., Lanerolle, L., Levin, J., McWilliams, J. C., Miller, A. J., Moore, A. M., Powell, T. M., Shchepetkin, A. F., Sherwood, C. R., Signell, R. P., Warner, J. C., Wilkin, J., 2008.

Regional Ocean Forecasting in Terrain-following Coordinates: Model Formulation and Skill Assessment, Journal of Computational Physics. 227, 3595-3624.

Haller, M. C., Dalrymple, R. A., 2001. Rip current instabilities. Journal of Fluid Mechanics, 433, 161192.

Haller, M. C., Dalrymple, R.A., Svendsen, I. A., 2002. Experimental study of nearshore 1044 dynamics on a barred beach with rip channels. Journal of Geophysical Research, 107(C6), 3061, 1045 doi:10.1029/2001JC000955.

Hasselmann, K., T.P. Barnett, E. Bouws, H. Carlson, D.E. Cartwright, K. Enke, J.A. Ewing, H. Gienapp, D.E. Hasselmann, P. Kruseman, A. Meerburg, P. M"uller, D.J. Olbers, K. Richter, W. Sell and H. Walden, 1973: Measurements of wind-wave growth and swell decay during the Joint North Sea Wave Project (JONSWAP), Dtsch. Hydrogr. Z. Suppl., 12, A8

Hughes, S.A., 1993. Physical Models and Laboratory Techniques in Coastal Engineering. World Scientific, Singapore.

Huntley, D.A., Short, A.D., 1992. On the spacing between observed rip currents. Coastal Engineering, 17, 211-225.

Jones, N.L., Monismith, S.G, 2008. The influence of whitecapping waves on the vertical structure of turbulence in a shallow estuarine embayment. Journal of Physical Oceanography, 38, 7, 1563-1580. 
Jenkins, A.D., 1989. The use of a wave-prediction model for driving a near-surface current model. Ocean Dynamics, Vol. 42., pp 133-146. doi: 10.1007/BF02226291

Kanarska, Y., Shchepetkin, A., McWilliams, J.C., 2007. Algorithm for non-hydrostatic dynamics in the Regional Oceanic Modeling System, Ocean Modelling, Volume 18, Issues 3-4, 2007, Pages 143-174, ISSN 1463-5003, 10.1016/j.ocemod.2007.04.001.

Kumar, N., Voulgaris, G., Warner, J.C., 2011a. Implementation and modification of a 3-D radiation stress formulation for surf zone and rip-current applications. Coastal Engineering, 58, 12, 1097-1117. doi: 10.1016/j.coastaleng.2011.06.009.

Kumar, N., Voulgaris, G., Warner, J.C., 2011b. Measurement and three-dimensional modeling of nearshore circulation on a South Carolina beach. Shore \& Beach, Vol. 79, 2, pp. 9-18.

Lai, Z., Chen, C., Cowles, G. W., Beardsley, R. C., 2010. A nonhydrostatic version of FVCOM: 1. Validation experiments, J. Geophys. Res., 115, C11010, doi: 10.1029/2009JC005525

Lane, E. M., Restrepo, J. M., McWilliams, J. C., 2007. Wave-current interaction: A comparison of radiation-stress and vortex-force representations, Journal of Physical Oceanography, 37, 1122-1141.

Lentz, S. J., Fewings, M., Howd, P., Fredericks, J., Hathaway, K., 2008. Observations and a model of undertow over the inner continental shelf. Journal of Physical Oceanography, 38, 2341-2357.

Longuet-Higgins, M.S., 1953. Mass Transport in Water Waves, Philosophical Transactions of the Royal Society of London, Series A 245: 535-581.doi: 10.1098/rsta.1953.0006.

Longuet-Higgins, M. S., Stewart, R.W., 1964. Radiation stresses in water waves: a physical discussion, with applications. Deep-Sea Research, 11, 529-562.

Longuet-Higgins, M. S., 1970. Longshore Currents Generated by Obliquely Incident Sea Waves. a, b, Journal of Geophysical Research. 75(33), 6778-6801.

MacMahan, J. H., Thornton, E. B., Stanton, T. P., Reniers, A. J. H. M., 2005. RIPEX: Observations of a rip current system, Mar. Geol., 218, 113-134.

Madsen, O.S., Y.-K. Poon and H.C. Graber, 1988: Spectral wave attenuation by bottom friction: Theory, Proceeding of 21st International Conference Coastal Engineering, ASCE, 492-504

Madsen, O.S., 1994. Spectral wave-current bottom boundary layer flows. In: Coastal Engineering 1994. Proceedings of the 24th International Conference on Coastal Engineering Research Council, Kobe, Japan, pp. 384-398.

McWilliams, J. C., Restrepo, J. M., Lane, E. M., 2004. An asymptotic theory for the interaction of waves and currents in coastal waters. Journal of Fluid Mechanics, 511, 135- 178.

Mellor, G. L., 2003. The three-dimensional current and surface wave equations. Journal of Physical Oceanography, 33, 1978-1989.

Mellor, G. L., 2005. Some consequences of the three-dimensional currents and surface wave equations. Journal of Physical Oceanography, 35, 2291-2298. 1079

Mellor, G. L., 2008. The depth-dependent current and wave interaction equations: A revision. Journal of Physical Oceanography, 38, 2587-2596. 
Mellor, G. L., 2011. Wave Radiation Stress. Ocean Dynamics, doi: 10.1007/s10236-010-0359-2.

Nairn, R.B., Roelvink, J.A., Southgate, H.N., 1990. Transition zone width and implications for modeling surf zone hydrodynamics. In: Proceedings of the 22nd International Conference on Coastal Engineering Delft, American Society of Civil Engineers, New York, 68-81.

Newberger, P.A., Allen, J.S., 2007a. Forcing a three-dimensional, hydrostatic primitive-equation model for application in the surf zone, 1: Formulation, Journal of Geophysical Research, 112, C08018.

Newberger, P.A., Allen, J. S., 2007b. Forcing a three-dimensional, hydrostatic, primitive-equation model for application in the surf zone: 2. Application to DUCK94, Journal of Geophysical Research, 112, C08019, doi:10.1029/2006JC003474.

Olabarrieta, M., Warner, J. C., Kumar, N., 2011. Wave-current interaction in Willapa Bay. J. Geophys. Res., 116, doi:10.1029/2011JC007387.

Phillips, O. M., The Dynamics of the Upper Ocean, 2nd ed., Cambridge Univ. Press, New York, 1977.

Rapp, R. J., Melville, W. K., 1990. Laboratory measurements of deep-water breaking waves. Phil.Trans. R. Soc. Lond. A 331, 735-800.

Reniers, A. J. H. M., Battjes, J. A., 1997. A laboratory study of longshore currents over barred and nonbarred beaches, Coastal Eng., 30, 1-22.

Reniers, A.J.H.M., Thornton, E.B., Roelvink, J.A., 2004a. Morphodynamic modeling of an embayed beach under wave-group forcing, Journal of Geophysical Research, 109, C01030, doi:10.1029/2002JC001586, 2004.

Reniers, A. J. H. M., Thornton, E. B., Stanton, T. P., Roelvink, J. A., 2004b. Vertical flow structure during Sandy Duck: Observations and modeling. Coastal Eng., 51, 237-260.

Reniers, A.J.H.M., MacMagan, J.H., Thornton, E.B., Stanton, T.P., Henriquez, M., Brown, J.W., Brown, J.A., Gallagher, E., 2009. Surfzone Surface Retention on a rip channeled beach, Journal of Geophysical Research, 114, C10010, 2009.

Ribas, F., de Swart, H.E., Calvete, D., Falqués, A., 2011. Modeling waves, currents and sandbars on natural beaches: The effect of surface rollers. Journal of Marine Systems, Vol. 88, 1, pp 90-101.

Ris, R.C., 1999. Model convergence of SWAN in the Westerschelde estuary. WL|Delft Hydraulics, Report H3496.

Roelvink, J.A., Reniers, A.J.H.M., 1994. LIP 11D Delta flume experiments; A dataset for profile validation. Delft Hydraulics report No. H2130.

Rogers, W.E., Hwang, P.A., Wang, D.W., 2003. Investigation of wave growth and decay in the SWAN model: three regional-scale applications, Journal of Physical Oceanography, 33, 366-389.

Ruessink, B. G., Miles, J. R., Feddersen, F., Guza, R. T., Elgar, S., 2001. Modeling the alongshore current on barred beaches. Journal of Geophysical Research, 106(C10), 22,451-22,463.

Shchepetkin, A. F., McWilliams, J.C., 2005. The Regional Oceanic Modeling System: A split-explicit, free-surface, topography-following coordinate oceanic model. Ocean Modeling, 347-404 doi:10.1016/j.ocemod.2004.08.002. 
Shchepetkin, A. F., McWilliams, J. C., 2009. Correction and commentary for "Ocean forecasting in terrain-following coordinates: Formulation and skill assessment of the regional ocean modeling system" by Haidvogel et al., Journal of Computational Physics, 227, 3595-3624.”. Journal of Computational Physics, 228, 8985-9000.

Sheng, P., Liu, T., 2011.Three-dimensional simulation of wave-induced circulation: Comparison of three radiation stress formulations. Journal of Geophysical Research, Vol. 116, C05021, doi: 10.1029/2010JC006765.

Short, A.D., 1985. Rip current type, spacing and persistence: Narrabeen Beach, Australia. Marine Geology, 65, 45-71.

Smith, J.A., 2006. Wave-current interactions in finite-depth, Journal of Physical Oceanography. 36, 1403-1419.

Soulsby, R.L., 1995. Bed shear-stresses due to combined waves and currents. In: M.J.F. Stive, Editor, Advances in Coastal Morphodynamics: An Overview of the G8-Coastal Morphodynamics Project, Co-Sponsored by the Commission of The European Communities Directorate General XII (1995), 4.204.23 .

Stacey, M. W., 1999. Simulations of the wind-forced near-surface circulation in Knight Inlet: A parameterization of the roughness length. J. Phys. Oceanogr., 29, 1363-1367.

Stive, M. J. F., De Vriend, H.J., 1994. Shear stresses and mean flow in shoaling and breaking waves. Proceedings of the 24th Coastal Engineering International Conference 1994, Kobe, Japan. ASCE, New York, 594-608.

Svendsen, I. A., 1984a. Wave heights and set-up in a surf zone, Coastal Engineering, 8, 303-329.

Tajima, Y., Madsen, O.S., 2006. Modeling near-shore waves, surface rollers and undertow velocity profiles. Journal of Waterways, Port, Coastal and Ocean Engineering ASCE, 132(6), 429-438

Ting, F.C.K., Kirby, J.T., 1994. Observations of undertow and turbulence in a laboratory surf zone. Coastal Engineering. 24, 177-204.

Thornton, E. B., Guza, R. T., 1983. Transformation of wave height distribution, Journal of Geophysical Research, 88, 5925-5938.

Uchiyama, Y., McWilliams, J.C., Restrepo, J.M., 2009. Wave-current interaction in nearshore shear instability analyzed with a vortex-force formalism, Journal of Geophysical Research, 114, C06021, doi:10.1029/2008JC005135.

Uchiyama, Y., McWilliams, J.C., Shchepetkin, A.F., 2010, Wave-current interaction in an Oceanic Circulation Model with a Vortex- Force Formalism: Application to the Surf Zone. Ocean Modeling, doi: 10.1016/j.ocemod.2010.04.002.

Umlauf, L., Burchard, H., 2003. A generic length-scale equation for geophysical turbulence models. Journal of Marine Research, 61, 235-265.

van der Westhuysen, A.J., Zijlema, M., Battjes, J.A., 2007. Nonlinear saturation-based whitecapping dissipation in SWAN for deep and shallow water. Coastal Engineering, Vol. 54, 2, pp. 151-170. 
Voulgaris, G., Collins, M.B., 2000. Sediment Resuspension on Beaches: Response to Breaking Waves. Marine Geology 167: 167-187.

Voulgaris G., Kumar, N., Warner, J.C., 2011. A Methodology for the Prediction of Rip Currents using a 3-D Numerical, Coupled, Wave-Current Model. In: Rip Currents: Beach Safety, Physical Oceanography, and Wave Modeling. S. Leatherman and J. Fletemeyer (Eds). CRC Press. ISBN: 9781439838969.

Walstra, D. J. R., Roelvink, J.A., Groeneweg, J., 2000. Calculation of wave-driven currents in a 1131 3D mean flow model. Proceedings of the 27th Coastal Engineering International Conference, 2000, Sydney, Australia. ASCE, New York, pp. 1050-1063.

Wang, J., Shen., Y., 2010. Modeling oil spills transportation in seas based on unstructured grid, finite volume, wave-ocean model. Ocean Model. 35 (4), 332-344.

Warner, J.C., Sherwood, C.R., Arango, H.G., Signell, R.P., Butman, B., 2005. Performance of four turbulence closure models implemented using a generic length scale method. Ocean Modeling. 8, 81-113.

Warner, J.C., Sherwood, C.R., Signell, R.P., Harris, C., Arango, H.G., 2008a. Development of a threedimensional, regional, coupled wave, current, and sediment-transport model. Computers and Geosciences. 34, 1284-1306.

Warner, J.C., Perlin, N., Skyllingstad, E.D., 2008b. Using the Model Coupling Toolkit to couple earth system models. Environmental Modeling and Software, Vol. 23, 10-11, pp 1240-1249.

Warner, J.C., Armstrong, B., He, R., Zambon, J.B., 2010, Development of a Coupled Ocean-AtmosphereWave-Sediment Transport (COAWST) modeling system: Ocean Modeling, v. 35, no. 3, p. 230-244.

Weber, J. E., G. Broström, and Ø Sætra, 2006: Eulerian versus Lagrangian approaches to the waveinduced transport in the upper ocean. Journal of Physical Oceanography, 36, 2106-2118.

Xu, Z., Bowen, A.J., 1994. Wave- and wind-driven flow in water of finite depth, Journal of Physical Oceanography, 24, 1850-1866.

Yu, J., Slinn, D. N., 2003. Effects of wave-current interaction on rip currents. Journal of Geophysical Research, 108(C3), 3088, doi:10.1029/2001JC001105.

Zijlema, M., Stelling, G., Smit, P., 2011. SWASH: An operational public domain code for simulating wave fields and rapidly varied flows in coastal waters, Coastal Engineering, Vol. 58, Issue 10, October 2011, pp. 992-1012, doi: 10.1016/j.coastaleng.2011.05.015. 